A fresh and frozen high-quality patient bio-sample is required in molecular medicine for the identification of disease-associated mechanisms at molecular levels. A common cooling procedure is immersing the tissue enclosed in a vial in a coolant such as liquid nitrogen. This procedure is not user-friendly and is laborious as reducing the lag time from excision time to freezing depends on the logistic organizational structure within a hospital. Moreover, snap freezing must be done as soon as possible after tissue excision to preserve the tissue quality for molecular tests.

In this thesis, starting from understanding the heat transfer mechanisms of different tissuefreezing procedures, an electrically powered snap freezing device as an alternative to quenching the vial in liquid nitrogen is presented. The device can be used directly at the location of tissue acquisition and also facilitates the study of the effect of freezing conditions on the various molecular processes in the samples.

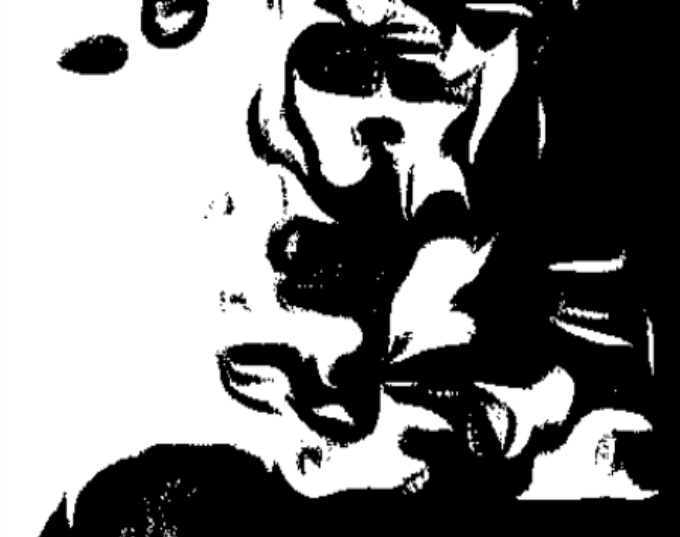

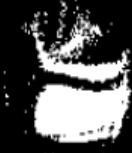

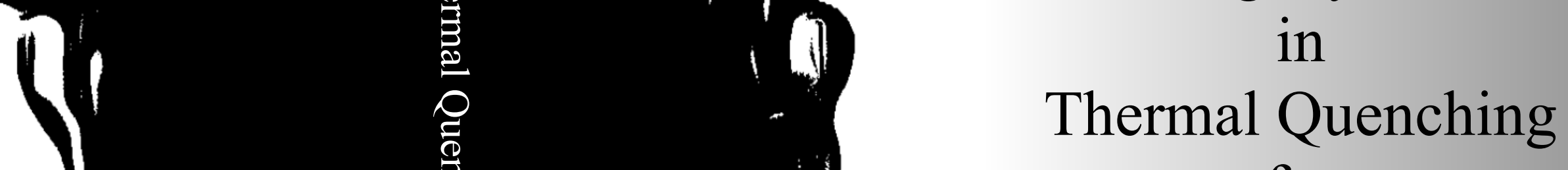
for

Cryopreservation

Sahil Jagga
Invitation

You are cordially invited to the defense of my doctoral thesis.

Cooling Dynamics in

Thermal Quenching for

Cryopreservation

The defense will be held online on Wednesday 16 th June 2021 at $14: 45$

Prior to the defense, I will give a short introduction at 14:30

Sahil Jagga

s.jagga@utwente.nl 


\section{COOLING DYNAMICS IN \\ THERMAL QUENCHING FOR CRYOPRESERVATION}

Sahil Jagga 


\section{Samenstelling promotiecommissie:}

Dr. ir. S. Vanapalli

Prof. dr. ir. H.J.M. ter Brake

Prof. dr. J.L. Herek

Prof. dr. ir. W.M. De Vos

Prof. dr. ir. D.W.F. Brilman

Prof. dr. ir. B. ten Haken

Prof. dr. -Ing. habil. Andrea Luke

Prof. dr. J. Pfotenhauer

Prof. Jean-Marie Buchlin
Universiteit Twente, assistant promotor

Universiteit Twente, promotor

Universiteit Twente, chairman

Universiteit Twente

Universiteit Twente

Universiteit Twente

Universität Kassel

University of Wisconsin-Madison

von Karman Institute for Fluid Dynamics

The work in this thesis was carried out at the Applied Thermal Sciences Lab, which is a part of EMS cluster, Faculty of Science and Technology, University of Twente. The Netherlands Organization for Scientific Research (NWO) is acknowledged for the financial support.

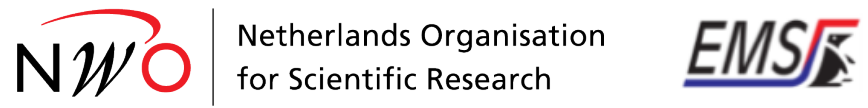

Publisher: Sahil Jagga, Applied Thermal Sciences Lab, EMS, University of Twente, P.O. Box 217, 7500 AE Enschede, The Netherlands

Cover: The front and back cover of the thesis shows artistic impression of an image captured during thermal quenching of an aluminum vial inside a liquid nitrogen pool.

(C)Sahil Jagga, Enschede, The Netherlands - 2021

No part of this work may be reproduced by print photocopy or any other means without the permission in writing from the publisher.

Printed by Ipskamp Printing, Enschede, Netherlands

ISBN: 978-90-365-5197-7

DOI: $10.3990 / 1.9789036551977$ 


\title{
COOLING DYNAMICS IN THERMAL QUENCHING FOR CRYOPRESERVATION
}

\section{PROEFSCHRIFT}

\author{
ter verkrijging van \\ de graad van doctor aan de Universiteit Twente, \\ op gezag van de rector magnificus, \\ Prof. dr. ir. A. Veldkamp \\ volgens besluit van het College voor Promoties \\ in het openbaar te verdedigen \\ op woensdag 16 juni 2021 om 14:45 uur
}

door

\section{Sahil Jagga}

geboren op 22 oktober 1991

te Haryana, India 
Dit proefschrift is goedgekeurd door:

Dr. ir. S. Vanapalli assistant promotor

Prof. dr. ir. H.J.M. ter Brake promotor 


\section{Dedication}

I am dedicating this thesis to my family members that include my parents (Arun Kumar and Deepshikha), brother (Sourabh), sister-in-law (Shweta), and my sweet little nephew (Suhaan). I am really thankful to them for being there at every stage of my life. Their support and love in my life have been unconditional and pure. I must admit that the teachings given by my parents have been the pillars of all my accomplishments so far. About my brother, I can not thank him enough for his immense support and care for my parents that allowed me to focus entirely on my work. This thesis in no way would have been possible without my family being there holding my hand. I am really thankful to God for giving me such a great family that has helped me in keeping the spirit high. 



\section{Contents}

1 Introduction 1

1.1 Introduction . . . . . . . . . . . . . . . . 1

1.2 Scope of the research . . . . . . . . . . . . . . 3

1.3 Scientific challenges ................ 4

1.4 Guide through the thesis ............... 8

Section - I : Thermal assessment of standard tissue snap-freezing pro$\begin{array}{ll}\text { cedures } & 11\end{array}$

2 What happens at the vial-liquid interface? 13

2.1 Introduction . . . . . . . . . . . . . . . 13

2.2 Heat transfer phenomenon . . . . . . . . . . . . 16

2.2.1 Heat transfer due to natural convection: . . . . . . . 18

2.2.2 Heat transfer due to boiling: . . . . . . . . . . . 18

2.3 Materials and methods ................ 21

2.4 Experimental results ................. 23

2.5 Heat transfer in isopentane and ethanol pool . . . . . . . . . . 24

2.6 Heat transfer in liquid nitrogen . . . . . . . . . . . . . . . 29

2.7 Conclusions .................... 30

3 How a polypropylene vial cools in liquid nitrogen? 31

Abstract ................... 31

3.1 Introduction . . . . . . . . . . . . . . . . 31

3.2 Experimental setup ................ 35

3.3 Heat transfer model and experimental validation . . . . . . . 37

3.3.1 Verification of PTCA . . . . . . . . . . . . 39

3.3.2 Approximate analytical model . . . . . . . . 39

3.4 Conclusions ..................... . . 47

Appendix - I . . . . . . . . . . . . . . . . . . 48

4 How to estimate minimum cool-down time for insulated metals in liquid nitrogen?

Abstract .................... 53 
4.1 Introduction . . . . . . . . . . . . . . . . 53

4.2 Materials and methods . . . . . . . . . . . . 55

4.3 Results and discussion . . . . . . . . . . . . . . 57

4.3.1 Phenomenological modeling . . . . . . . . . . . 62

4.3.2 Validation of assumptions in the model . . . . . . . 68

4.4 Conclusions . . . . . . . . . . . . . . . . 71

Appendix - II . . . . . . . . . . . . . . . 71

Section - II : Development of a tissue snap-freezer without sacrificial $\begin{array}{ll}\text { cryogens } & 75\end{array}$

5 Tissue snap-freezer: design 77

Abstract . . . . . . . . . . . . . . . . . 77

5.1 Systematic design . . . . . . . . . . . . . . . 77

5.1 .1 Cooling concepts . . . . . . . . . . . 78

5.2 Design methodology . . . . . . . . . . . . . . 82

5.3 Model . . . . . . . . . . . . . . . . . . 88

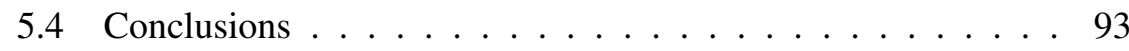

Appendix - III . . . . . . . . . . . . . . . . . . . . . . . . . 994

6 Tissue snap-freezer: measurements and model validation 99

Abstract . . . . . . . . . . . . . . . . . . . . 99

6.1 Materials and methods . . . . . . . . . . . . . . . . 99

6.2 Results and Discussion . . . . . . . . . . . . . . . 101

6.2.1 Cooling affect by thermal diffusion . . . . . . . . . 101

6.2.2 Vial cool-down with different heat sink temperature . . 103

6.2.3 Vial cool-down with various gases in the snap-freezer . 104

6.2.4 Vial cool-down with different mass flow rate in the snap-freezer . . . . . . . . . . . . . . 105

6.3 Numerical methods . . . . . . . . . . . . . . . . . . . . 107

6.4 Conclusions . . . . . . . . . . . . . . . . 111

7 Systematic approach to determine the transient cooling power and heat leak of a commercial pulse tube cryocooler. 113

Abstract . . . . . . . . . . . . . . . . . . 113

7.1 Introduction . . . . . . . . . . . . . 113

7.2 Materials and procedure . . . . . . . . . 115 
7.3 Results and discussion . . . . . . . . . . . . . . . 117

7.3.1 Effective cold mass at the cold heat exchanger . . . . . 117

7.3.2 Steady and transient cooling power . . . . . . . . 118

7.3.3 Background heat losses . . . . . . . . . . . . . . 123

7.3.4 Cool-down of an added mass to the cold-end . . . . 129

7.4 Conclusions . . . . . . . . . . . . . . . 131

8 Conclusions and Outlook 133

8.1 Conclusions . . . . . . . . . . . . . . . . . 133

8.2 Outlook . . . . . . . . . . . . . . . 135

$\begin{array}{ll}\text { Appendix } & 139\end{array}$

$\begin{array}{ll}\text { Summary } & 143\end{array}$

$\begin{array}{ll}\text { Samenvatting } & 147\end{array}$

$\begin{array}{ll}\text { Acknowledgment } & 151\end{array}$

$\begin{array}{ll}\text { About the author } & 155\end{array}$ 



\section{CHAPTER 1 \\ Introduction}

\subsection{Introduction}

Humans have always known that low temperatures preserve biological constructs naturally. In Palaeolithic times, the depths of the caves were both a clever hiding spot and an ideal place to store food cold. In modern days, refrigerators and freezers are used for the same purpose; these methods slow down or stop most bacteria from dividing and thereby multiplying, but do not kill them. Food in the refrigerators can be preserved for days, whereas freezing at $-18{ }^{\circ} \mathrm{C}$ and deep freezing at $-40{ }^{\circ} \mathrm{C}$ allows the food to be preserved for several months. For longer duration preservation (usually years), cryogenic freezing is generally used; it is a technique to preserve by cooling to temperatures below the glass transition temperature $\left(T_{g}\right)$ of water, approximately -136 ${ }^{\circ} \mathrm{C}[1]$, where any enzymatic or chemical activity that may cause harm to the biological construct is essentially stopped, and the frozen material may thus have an infinite lifetime. However, the actual effective life is still unknown and is rather difficult to prove.

Cryopreservation for human specimens was implemented in 1954 with three pregnancies stemming from previously frozen sperm insemination [2]. Later in 1957, a group of UK scientists headed by Christopher Polge preserved a fowl sperm [3]. Cryo-preservation is also used for many applications such as preserving embryos, ovarian tissues, oocytes, testicular tissues, moss, microbiology cultures such as fungi, bacteria, and worms, etc. As the world population has undergone exponential growth, it is predicted to increase from current 7.5 billion to 9.7 billion by 2050 [4]; with this substantial human population growth, the demand for cryo-preserved bio-specimens would increase significantly. So far, the collection of frozen bio-specimens has primarily been the domain of research work, but "next-generation" testing is increasingly moving into everyday clinical care, indicating that frozen tissue collections may become standard when cancer or certain disorders are suspected. In the coming 
years, pathology departments and bio-banks will undoubtedly have to store and disseminate a growing number of frozen bio-specimens. Furthermore, the increasingly affordable "next-generation" technology has evolved quickly, enabling global or targeted assessment of tissue and cell genome, epigenome, proteome, and metabolomes, and which is key to personalized medicinestailoring targeted treatments for each patient.

For tissue preservation, two methods are usually used - chemical fixation or physical fixation, also known as cryo-preservation. In chemical fixation, a tissue sample is first preserved by fixing it in formaldehyde, also known as formalin, to preserve the proteins and vital structures within the tissue. Next, it is embedded in a paraffin wax block; this makes it easier to cut slices of required sizes to mount on a microscopic slide for examination. On the other hand, for cryo-preservation, a cryo-vial is usually used to contain the excised tissue, which is then immersed in a coolant. Ultra-low temperature frozen tissue and the tissue embedded with formalin-fixed paraffin each have their advantages and disadvantages [5-7]. Histology of frozen tissue is often adequate for quality assurance, but for detailed microscopic analyses, usually, FFPE tissue is favored. However, the DNA and RNA from frozen bio-specimens have relatively high molecular weight without cross-linking and, therefore, are suitable for a wide variety of purposes. The DNA and RNA quality of the frozen-tissue is ideal for current approaches such as whole genome-amplification, whole genome-sequencing, and cDNA microarray analyses [8, 9]. Unlike FFPE, in the frozen-state, proteins are uniquely well preserved, including enzymatic activity [10]. At ultra-low temperatures, biological constructs can be stored up to several years. However, studies have shown that RNA fragmentation after five years despite storage at low temperatures $[11,12]$. To summarize, cryopreserved tissue is the preferred bio-specimen for modern testing as it generates high performance and high-quality nuclear acids and proteins compared to the formalin-fixed paraffin (FFPE) tissue [8].

Currently, for tissue cryo-preservation by freezing, a cryo-vial made up of either aluminum or polypropylene material is usually used to contain the excised tissue, which is then immersed in a coolant such as saturated liquid nitrogen or sub-cooled isopentane: pre-cooled to approximately $113 \mathrm{~K}$ using liquid nitrogen [13]. The heat transfer phenomenon at the vial-liquid interface varies strongly with the thermal properties of the vial material and the 
liquid thermodynamic state; the effect of these parameters on the heat transfer rate at the vial-liquid interface is not well understood and therefore, a random procedure is usually followed: immersing the vial for a random duration that varies across institutions. The variation between the used freezing procedures results in different freezing rates, which might lead to having different quality of the similar tissues frozen using different snap-freezing procedures, as also found by Baucamp et al. in their study [14]. The difference in the quality is suspected to be due to different freezing rates of the water present inside the tissue.

During the freezing process, ice-crystals are usually formed, which often leads to cellular mechanical constraints and injuries [15], and thus may cause great harm to the frozen tissue. Injuries corresponding to fast cooling rates are due to intracellular ice formation, whereas slow cooling results in increased exposure to highly concentrated intra and extracellular solutions that causes osmotic changes in the tissue. For the future development, and to find the safest and most effective freezing procedures, a better understanding of the heat transfer aspects for these procedures is therefore required; this will play a key role in therapeutic utility-related research in all kinds of human trials.

\subsection{Scope of the research}

So far, it is not known what the cooling rate of the tissues would be when vials are immersed in a coolant. As discussed before, the cooling procedure has an impact on the morphology of the specimen enclosed. During the freezing procedure, the vial material, together with the coolant used, influences the cooling rate at the vial-liquid interface. As the heat transfer to the tissue occurs through the vial wall into the liquid, the heat transfer at the vial wall influences the cool down of the tissue and, therefore, its quality in the frozen state. A systematic and quantitative study to understand the heat transfer aspects of these procedures is lacking and needs to be addressed urgently. This would help to standardize the existing and developing novel freezing procedures.

The conventional freezing procedures also have other limitations. The usage of liquid nitrogen is not allowed inside the operation theater; due to which the tissue is frozen at a different location from its excision; this increases the lag time between the tissue excursion and its freezing, which also degrades the 
tissue quality, as tissue freezing must be done immediately after its excursion [16]. Additionally, the availability of liquid nitrogen requires managing large infrastructure and trained personnel for its maintenance. Therefore, alongside understanding the existing snap-freezing procedures, there is a need to provide an improved alternative in the form of an apparatus to overcome the limitations discussed earlier.

The commercially available freezing devices are discussed now; the cooling rate of Mr. FrostyTm freezing container is maintained at $-1 \mathrm{~K} / \mathrm{min}$ to a temperature of $193 \mathrm{~K}$ [17]. CoolRack uses dry ice and can cool the vials to $193 \mathrm{~K}$ in 1-2 minutes [18]. The HistoChill uses 3M Novec 7000 as a liquid coolant bath cooled with a mechanical refrigerator [19], using these higher freezing rates compared to CoolRack is reported. Stand-alone Gentle Jane is a portable device that uses liquid nitrogen with a controllable freezing rate [20]. The achievable cooling rates for all these devices is very low and, therefore, cannot be used for the discussed snap freezing procedures.

An electrically operated apparatus that allows snap freezing of the tissue to different end temperatures is therefore required. For freezing the tissues, the device should not release any gaseous cryogens, so that it can be allowed to use inside the operation theater as this will help to reduce the lag time between the tissue excision and freezing. The apparatus should also have additional features to facilitate research on the impact of freezing characteristics on the viability of the tissue. The desired features of the apparatus are listed below:

- Adjustable cold sink temperature between $80 \mathrm{~K}$ to $200 \mathrm{~K}$.

- Adjustable cooling rates faster than the liquid nitrogen quenching rate.

- Limited venting of gases in-line with the safety norms of the operation theatre.

- Commonly used cryo-vials can be used.

\subsection{Scientific challenges}

\section{Heat transfer in the vial wall and at the vial-liquid interface for conven-}

tional freezing procedures: As discussed previously, the tissue during the snap-freezing process is placed at the inner wall of the vial (see Figure 1.1) and therefore, the heat transfer from the tissue occurs through the vial-wall 
into the cold fluid. Estimating the heat transfer coefficient $(h)$ at the vialliquid interface and the heat transfer rate in the vial-wall is necessary for the cool-down estimation of the tissue. The challenges in understanding these aspects include the following:

1. Non-standardized cryo-vials: The cryo-vials size and shape used for the tissue snap-freezing are non-standardized. The heat transfer coefficient $(h)$ values in both liquid nitrogen [21-25] and isopentane [26, 27] is a function of several parameters that also includes the shape and dimensions of the object to be cooled. Therefore, for vials of different sizes and shapes, the heat transfer coefficient $(h)$ values will be different, resulting in different cooling rates. Additionally, these correlations for estimating the heat transfer coefficient are derived using steady-state measurements and, therefore, are not valid for the transient cool-down conditions.

2. Uncontrolled cryo-vial immersion process: The heat transfer coefficient values in isopentane [26,27] and liquid nitrogen [28] are also dependent on cryo-vial orientation. As the vial insertion into the liquid is done manually and randomly, this leads to having an uncontrolled orientation of the vial inside the liquid pool resulting in unpredictable heat transfer coefficient $(h)$ values. Also, convective currents caused during the vial immersion into the pool results in increased heat transfer coefficient $(h)$ values at the vial-liquid interface, which can not be estimated as the insertion process is non-repetitive.

3. Temperature-dependent thermal properties: The thermal properties of the vial materials used (aluminum [29-31] and polypropylene [32, 33]) strongly varies with temperature; this should be taken into account while estimating the heat transfer rate inside the vial material.

Addressing these challenges to come up with a generalized heat transfer model for estimating the cool down of the tissue enclosed in a cryo-vial requires a good thermal understanding of the heat transfer at the vial-liquid interface and also within the vial material. Sansinena et al. [34] studied the effect of the external heat transfer coefficient on the cooling rates of the liquid-filled polypropylene straw, which is plunged directly into liquid nitrogen. For this purpose, the unsteady-state heat conduction equation for concentric cylinders 


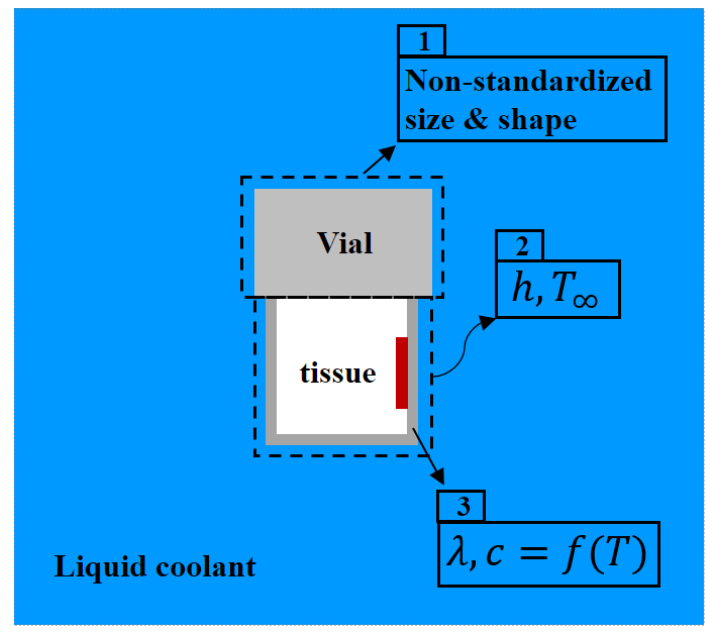

Figure (1.1) Thermal interaction of the vial with the cold liquid at temperature, $T_{\infty}$ with limited heat transfer coefficient, $h$. The numbers 1,2 and 3 indicate the scientific challenges.

was numerically solved using the external heat transfer coefficient as a fitting parameter. Jiang et al. [35] computed the convective heat transfer coefficient on the outer wall of the cryovial being plunged into liquid nitrogen using an inverse problem method. Wang et al. [36] used the natural convection heat transfer model to estimate the heat transfer coefficient values using measurements of the cryovials filled with CPA solution being plunged into liquid nitrogen; to do this, they used the heat transfer coefficient as a fitting parameter to match their calculation with the measurements. In conclusion, the studies reported so far as to estimate the cool-down of the vials during snap-freezing are empirical and do not assume temperature-dependent properties for the vial material. Therefore, the quantitative information on the heat transfer process at the vial-liquid interface and inside the vial wall is still a mystery limiting the cryo-vial and enclosed tissue's cool-down prediction.

Understanding heat transfer aspects of material like polypropylene in liquid nitrogen also opens up the possibility to understand a well known yet baffling phenomenon where the cooling rate of a metallic object quenched in liquid nitrogen can be enhanced by coating its surface with a material that has thermal effusivity value of the same order of polypropylene. This is useful to many applications that aspire faster cool-down; this includes quenching tool steel 
in a liquid nitrogen bath to improve hardness [37], various cryogenic systems where the connecting pipelines must be first chilled [38, 39].

Previous studies on the cool down of coated metals in liquid nitrogen [4044] suspect an early transition from film to nucleate boiling regime caused by the formation of cold spots at the liquid-coating interface. However, no microscale heat transfer studies are reported to support this claim. From an application perspective, it is clear that an insulation coating decreases the cool-down time. However, a tool to predict an optimum coating thickness based on the material's thermal properties is still lacking. The scientific challenge in predicting the cool-down of coated materials in liquid nitrogen are the unknown heat transfer coefficient values at its surface in contact with the liquid.

Development of tissue snap-freezer: The tissue snap-freezer is developed by assessing different cooling principles to meet the device requirements. As an output to the assessment, the forced convective cooling method is selected as the cooling principle; using this, the vial is cooled using a thin gas-gap between the vial and a cold heat capacity called Thermal Energy Storage Unit (TESU) cooled using a commercial off-the-shelf pulse-tube cryocooler (see Figure 1.2).

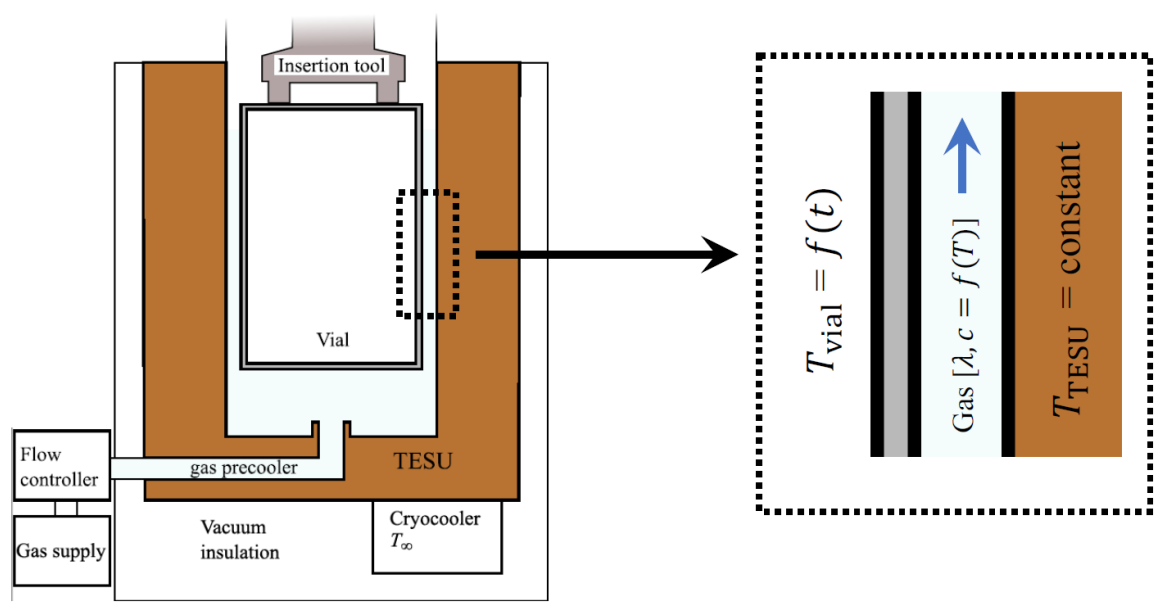

Figure (1.2) Conceptual design of the snap-freezing apparatus showing its main functional components. 
Due to the sub-mm gas-gap size, the gas flow in the gas gap of the snapfreezer can be considered as the fluid flow between two parallel plates. The solutions predicting transient temperature profiles in the fluid domain are available for constant wall temperature values, for which thermal properties of the fluid are assumed constant. These solutions can not be used for our case, as the temperature of one of the walls (vial) is time-dependent, and the thermal properties of the gases used (helium, nitrogen, and heliox) are temperature dependent. The scope of research here is, therefore, to model thermal interaction of the vial with the TESU through the gas-gap.

The preparation time for the tissue snap-freezer is determined mainly by the time to cool-down the attached heat capacity (TESU). In a typical pulse tube cryocooler application, modeling the cool-down dynamics of a load attached to the cold tip requires knowledge of the transient cooling power. Although this data may be calculated for in-house developed cryocoolers, the only data provided in commercial off-the-shelf cryocoolers is the steady-state cooling power. Another scope of the research here is, therefore, to show an approach to determine the transient cooling power and the corresponding parasitic heat leaks of a commercial off-the-shelf pulse tube cryocooler.

\subsection{Guide through the thesis}

The thesis is divided into two sections (see Figure 1.3). Section-I discusses the thermal assessment of the standard tissue snap-freezing procedures. SectionII discusses the development of a tissue snap-freezer. The outline of these sections is discussed below.

\section{Section-I: Thermal assessment of standard tissue snap- freezing procedures}

Chapter 2 discusses the phenomenological understanding of the heat transfer process at the vial-liquid interface during thermal quenching in the liquid coolants used for snap-freezing. To do this, experiments are performed to determine the cooling rate of standard cryo-vials in commonly used coolants such as liquid nitrogen and cold isopentane. Chapter 3 discusses an analytical model to predict temperature variation inside the polypropylene vial-wall and of the tissue attached to it. This model also includes temperature-dependent 


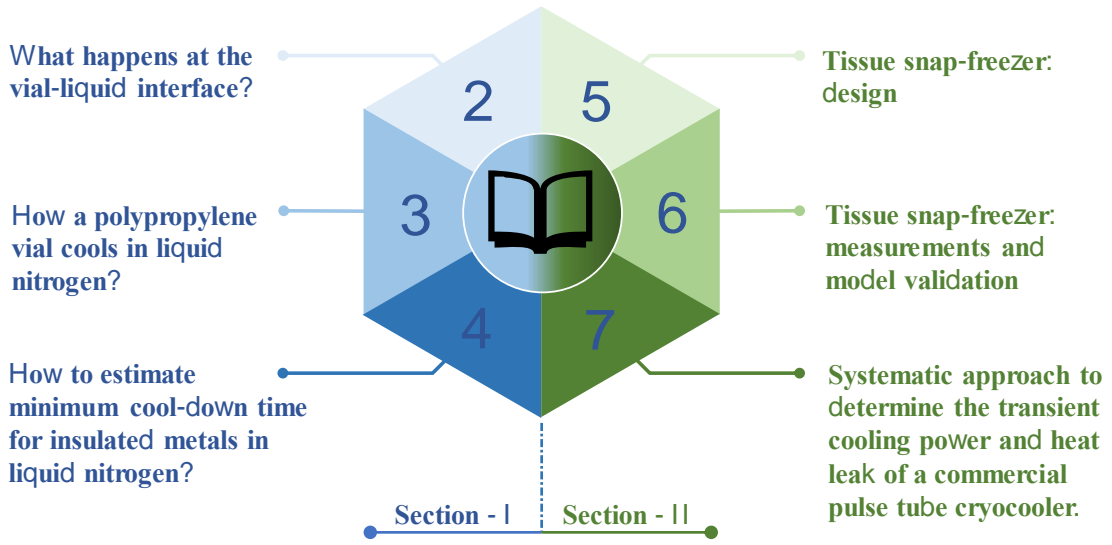

Figure (1.3) Overview of the thesis main chapters

thermal properties of the polypropylene material. An interesting finding from this work is that the difference in the cool-down time between tissue in a tube and in an empty tube is proportional to the product of the tissue heat capacity and the tube-wall thermal resistance. This enables researchers to estimate the cooling trajectory of the tissue during the cooling process and thus allowing the development of improved and more precise snap-freezing protocols.

From the output of the study reported in Chapter 3, it was found that during cool-down in liquid nitrogen, the Perfect Thermal Contact Assumption (PTCA) at the liquid-solid interface is valid for the materials with thermal effusivity as low as polypropylene material. This inspired us to develop an experimental approach to predict the minimum cool-down time for the metals coated with epoxy of low thermal effusivity material, which is reported in Chapter 4. We also discussed the effect of sub-cooled liquid nitrogen on the minimum cool-down time of coated metals. The approach discussed is also validated using measurements performed by quenching a metallic cylinder coated with different thicknesses of epoxy material (stycast 1266) inside a liquid nitrogen bath. The outcome of this study will be useful when designing cryogenic systems where faster cooling speed during quenching in liquid coolants is desired. For example, a new type of cryo-vials that cools faster than the conventional type made of aluminum or polypropylene material can be designed. 


\section{Section-II: Development of a tissue snap-freezer with- out sacrificial cryogens ${ }^{1}$}

In Chapter 5, the design methodology of a tissue snap-freezing apparatus, which is powered by a cryo-cooler and uses forced convective gas-flow to cool the vial, is discussed. A mathematical model is also developed to capture the cooling dynamics of a cryo-vial in the snap-freezer. Several parameters to control the cooling rate of the vial are also discussed. In Chapter 6, the measurements performed with the developed snap-freezer are discussed and compared with the predicted values using the heat transfer model.

In Chapter 7, the transient characteristics of a commercial cryo-cooler used in the snap-freezer are discussed. A novel experimental approach to calculate parasitic heat leaks and the cooling power for both steady-state and transient conditions are discussed. The calculated cooling power values are also verified using measurements. The output of this study allows us to estimate the cool-down time of the developed snap-freezer.

\footnotetext{
${ }^{1} \mathrm{~A}$ very small amount of gas vents out to avoid moisture accumulation during the cooldown of the system, which is in-line with the safety regulations of the hospitals
} 


\section{Section - I}

\section{Thermal assessment of standard tissue snap-freezing procedures}




\section{What happens at the vial-liquid interface?}

\section{Abstract}

Snap-freezing of tissue is commonly used for rapid intraoperative diagnosis and long term cryo-preservation, for which a good quality frozen-tissue is essential. For freezing the tissue, a common method is to place the tissue in a vial, followed by immersing the vial in a coolant. Various snap-freezing procedures use different vials and coolants, resulting in varied cooling rates for the tissue. A study reported by Steu et al. [14] concludes that a similar tissue frozen using different snap-freezing procedures show different quality; therefore, known temperature variation of tissue for different snap-freezing procedures would be helpful for the clinical researchers to adopt best and design more precise cooling procedures for better quality frozen-tissue. In this chapter, we systematically measured the cooling rates for different snapfreezing procedures. From the measurements, we found that a polypropylene vial cools faster in the liquid nitrogen than cold isopentane and ethanol bath whereas, the opposite is true for the case of the aluminum vial. We also observed that the boiling regime around the vials made up of high (aluminum) and low thermal effusivity (polypropylene) materials are different.

\subsection{Introduction}

A common tissue snap-freezing procedure involves enclosing the tissue inside a cryo-vial and subsequently immersing the vial into the coolant. During the first contact of a vial with the coolant, the outer surface of the vial begins to drop in temperature. Gradually, this thermal disturbance diffuses through the vial wall to the enclosed tissue. The heat transfer at the vial-liquid interface, therefore, influences the cooling of the tissue. The cooling rate of the tissue is very important for its quality in the frozen state, as reported by Baucamp et 
al. [14]; in which identical tissues snap-frozen using different snap-freezing procedures results in significantly different tissue quality. Known values of the tissue cooling rates obtained with different snap-freezing procedures will therefore, help estimate the tissue quality and subsequently adapting the best cooling procedure to obtain good quality tissue; this can be accomplished by developing a quantitative understanding of the heat transfer phenomena at the vial-liquid interface as the cooling of the tissue occurs due to heat transfer at this interface.

Commonly used cryo-vials for tissue snap-freezing are made up of polypropylene material, and few are made up of aluminum. The coolants used are liquid nitrogen, cold isopentane, and cold ethanol [13]. For the snap-freezing procedure, isopentane is pre-cooled to its freezing point of approximately $113 \mathrm{~K}$ using liquid nitrogen, and ethanol is pre-cooled using dry-ice to the temperature of approximately $200 \mathrm{~K}$. Sansinena et al. [34] studied the effect of the external heat transfer coefficient on the cooling rates of the liquid-filled polypropylene straw which is plunged directly into liquid nitrogen. For this purpose, the unsteady-state heat conduction equation for concentric cylinders was numerically solved using the external heat transfer coefficient as a fitting parameter. Wang et al. [36] used the natural convection heat transfer model to estimate the heat transfer coefficient values using measurements of the cryovials filled with CPA solution being plunged into liquid nitrogen; to do this, they used the heat transfer coefficient as a fitting parameter to match their calculation with the measurements. The same study is then extended by Jiang et al. [35] to predict transient temperature variation of the bio-materials enclosed in the vial and quenched in liquid nitrogen. To do this, the convective heat transfer coefficient on the outer wall of the cryo-vial during plunging into liquid nitrogen was computed using an inverse problem method. Both these studies $[35,36]$ conclude that the heat transfer at the vial-liquid interface occurs due to film boiling followed by nucleate boiling; the temperature of the cryo-vial and the enclosed tissue can be best predicted by assuming a heat transfer coefficient value of approximately $35 \mathrm{Wm}^{-2} \mathrm{~K}^{-1}$ and $550 \mathrm{Wm}^{-2} \mathrm{~K}^{-1}$ for film and nucleate boiling, respectively. All these studies are empirical and do not provide a good understanding of the heat transfer phenomenon for developing a generalized heat transfer model, which is independent of various parameters such as vial shape, dimensions, and material thermal properties, to predict the 
temperature of the tissue enclosed. Sansinena et al. [34] did not validate their numerical model with measurements. The studies $[35,36]$ claiming the existence of film boiling regime for polypropylene materials are also incorrect as for materials with thermal effusivity values as low as a polypropylene material, the film boiling regime does not exist and is replaced with so-called larvate boiling regime [40]. Also, in all these studies, the thermal properties of the polypropylene material are assumed temperature independent, which is incorrect. The heat capacity of polypropylene varies significantly in the temperature range of $295 \mathrm{~K}$ to $77 \mathrm{~K}$ (see Figure 2.1) which, therefore, will have a significant effect on the heat transfer rate from the tissue; as the heat transfer occurs through the vial-wall into the liquid coolant.

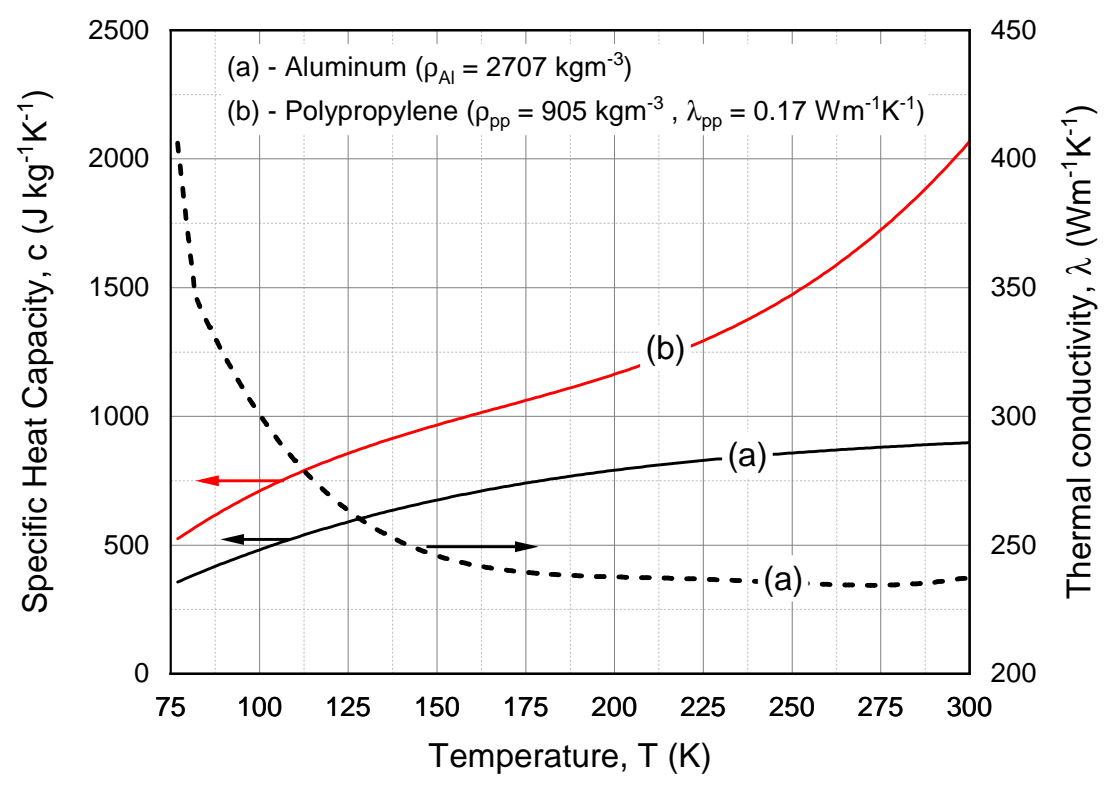

Figure (2.1) Temperature dependent thermal properties of aluminum [29$31]$ and polypropylene material $[32,33]$. The thermal conductivity of the polypropylene material is fairly constant for the temperature range of $77 \mathrm{~K}$ to $295 \mathrm{~K}$. 
To summarize, a study reporting detailed understanding of the heat transfer aspects for different cooling procedures is lagging and needs to be addressed; the study must address the effect of vial thermal properties and the coolant thermodynamic state on the heat transfer rate at the vial-liquid interface, temperature variation inside the vial-wall and of the tissue attached to it. This chapter addresses the need and reports quantitative cooling rates obtained at the inner wall of both the cryo-vials for different snap-freezing procedures. To do this, a systematic set of experiments is performed to measure the temperature variation of the cryo-vials during its cool down. We also report a phenomenological understanding of the heat transfer at the vial-liquid interface for a different combination of the liquid-solid material used in these snapfreezing procedures; this would be helpful in developing a heat transfer model for predicting temperature variation inside the vial-wall and of the tissue attached to it.

\subsection{Heat transfer phenomenon}

A brief introduction to the thermodynamic properties of coolants in the liquid state is presented here as an aid in the further development of the heat transfer process between the coolant and the vial. The difference in the coolant thermodynamic states can be better understood by looking at the schematic representation of a typical pressure-temperature $(P-T)$ diagram of a substance shown in Figure 2.2a. The boiling temperature of liquid nitrogen at $1 \mathrm{~atm}$ is $77.36 \mathrm{~K}$ and, therefore, when a vial at room temperature is inserted, the liquid nitrogen changes its state from liquid to the vapor state, as can be seen in the schematic representation of pressure-enthalpy $(P-h)$ diagram for liquid nitrogen. Isopentane is pre-cooled to its freezing point of approximately 113 $\mathrm{K}$, the boiling temperature $T_{\text {boiling }}$ of the isopentane at $1 \mathrm{~atm}$ is $301 \mathrm{~K}$, which is above the immersion temperature of the vial $(\approx 295 \mathrm{~K})$. Isopentane, therefore, remains in the liquid state during the entire cooling process. The ethanol bath is pre-cooled using dry-ice to the temperature of approximately $200 \mathrm{~K}$ and therefore remains in the liquid state, as the freezing point of ethanol is approximately $159 \mathrm{~K}$ and its boiling temperature $T_{\text {boiling }}$ at $1 \mathrm{~atm}$ is $351 \mathrm{~K}$, which is above the immersion temperature of the vial $(\approx 295 \mathrm{~K})$. 


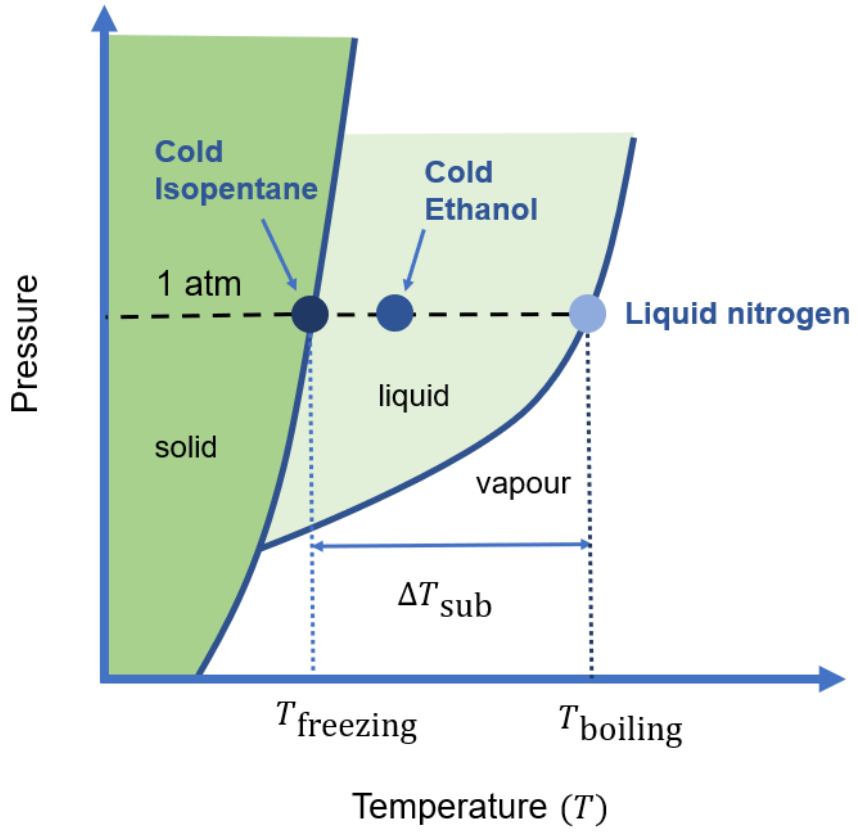

(a)

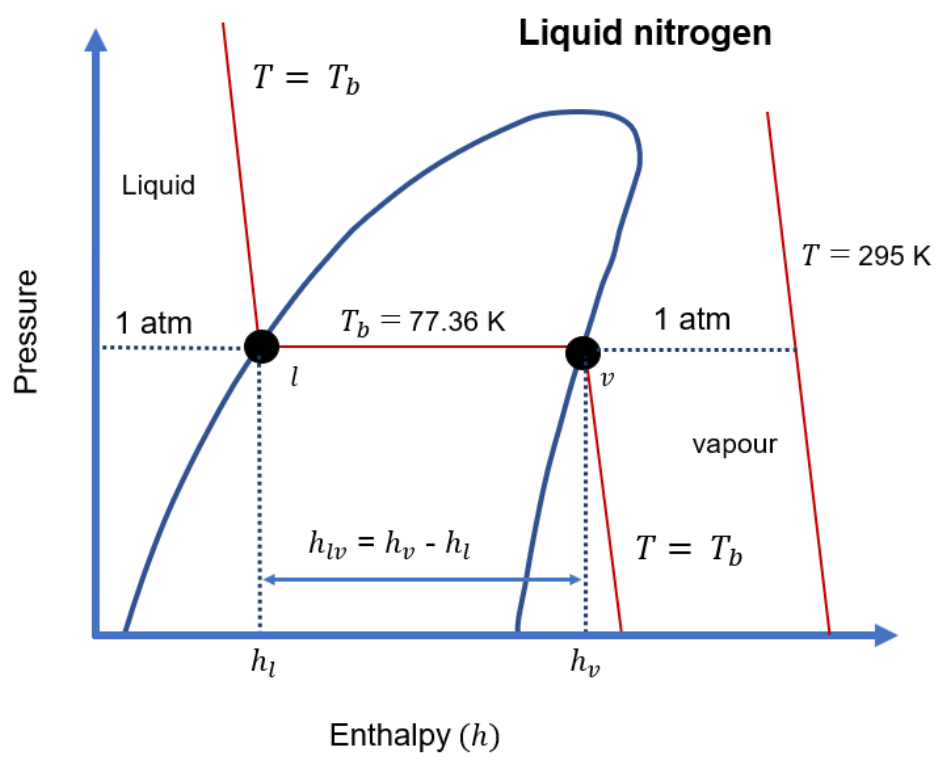

(b)

Figure (2.2) (a) Typical $P-T$ (pressure-temperature) diagram of a liquid. (b) Schematic representation of $P-h$ (pressure-enthalpy) diagram for liquid nitrogen. 
Now, a brief introduction to the heat transfer in isopentane and ethanol, which occurs due to the natural convection, and in liquid nitrogen that occurs due to boiling is presented below.

\subsubsection{Heat transfer due to natural convection:}

In natural convection, fluid flow is caused by buoyancy. The buoyancy effect results from the general tendency of fluids to expand (not always) when heated at constant pressure. The temperature of the fluid increases as it comes in contact with the warm object, thus making it lighter compared to the rest of the fluid. This causes fluid to flow upward and simultaneously, collecting heat from the wall in contact. Figure 2.3 shows the schematic of the fluid flow and the pressure distribution during the natural convection heat transfer along a vertical wall.
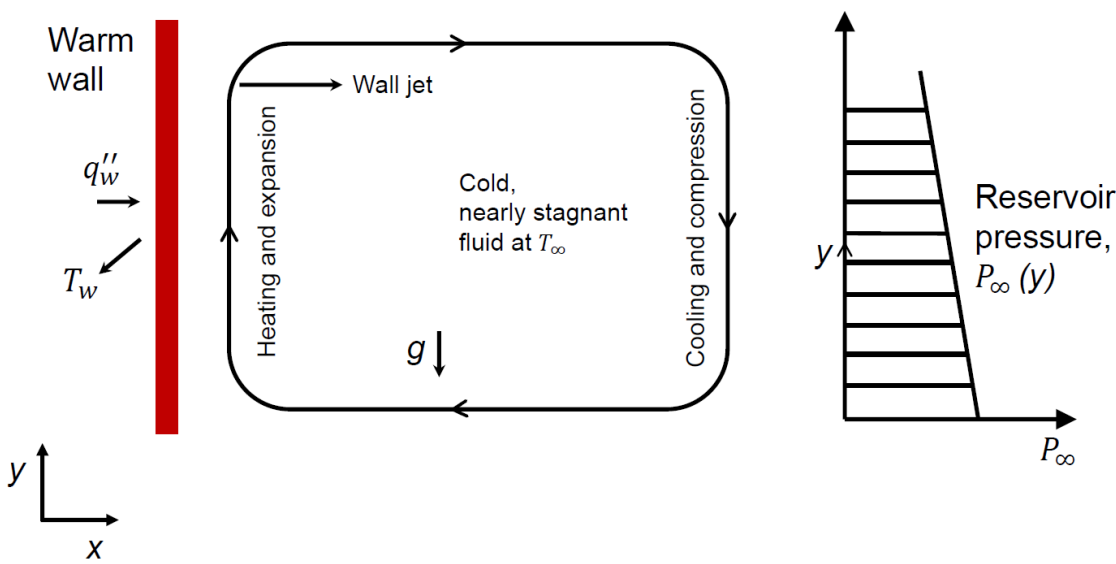

Figure (2.3) Fluid flow drive by buoyancy along a heated wall and pressure distribution in the reservoir of stagnant fluid [45].

\subsubsection{Heat transfer due to boiling:}

A typical pool boiling curve, showing qualitatively the dependence of the wall heat flux $(q)$ on the wall superheat $\left(\Delta T=T_{\text {wall }}-T_{\text {sat }}\right)$ is shown in the Figure 2.4. The pool boiling curve is divided into four different regimes, namely, film (section DE), transition (section $\mathrm{CD}$ ), nucleate (section $\mathrm{BC}$ ), and natural convection (section $\mathrm{AB}$ ), which are discussed next. 

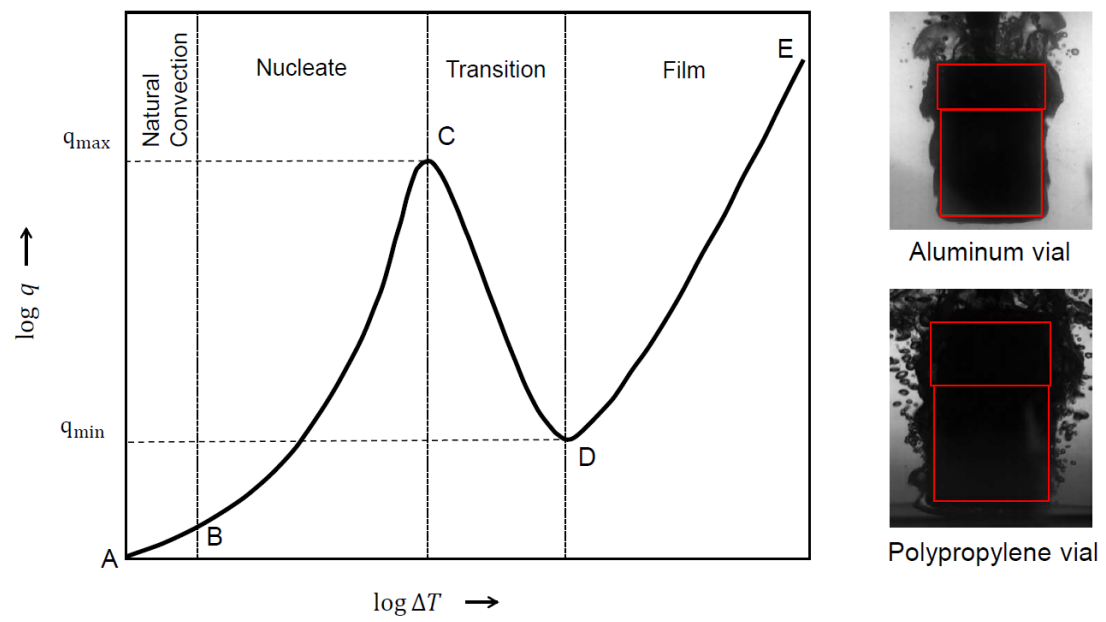

Polypropylene vial

Figure (2.4) Typical boiling curve, showing qualitatively the dependence of the wall heat flux $(q)$ on the wall superheat $\left(\Delta T=T_{\text {wall }}-T_{\text {sat }}\right)$ [46]. Section $\mathrm{AB}, \mathrm{BC}$ and $\mathrm{CD}$ represents the nucleate, transition and film boiling regime, respectively. Boiling phenomena captured at the start of the quenching in liquid nitrogen is shown at the right.

Film boiling regime occurs at the start of the quenching process i.e., high wall superheat ( $\Delta T=T_{\text {wall }}-T_{\text {liquid }}$ ), where the object to be cooled is covered with a vapor blanket, that adds resistance to the heat transfer from the object to the liquid. The film boiling regime is significant in studying the heat transfer during quenching in a liquid pool as it covers most of the temperature range to be cooled; for an object at room temperature immersed in liquid nitrogen, it occurs for approximately $87 \%$ ( $295 \mathrm{~K}$ to $105 \mathrm{~K}$ ) of the entire temperature range ( $295 \mathrm{~K}$ to $77 \mathrm{~K}$ ). The vapor film around the object breaks when the wall superheat $\left(\Delta T=T_{\text {wall }}-T_{\text {sat }}\right)$ drops below a certain value, as the rate of vapor generation eventually becomes too small to sustain the vapor film and the boiling regime then goes through a transition from film to the nucleate boiling. The temperature of the liquid-solid interface corresponding to this transition (Point "D" in Figure 2.4) is called the minimum film boiling temperature (MFBT), which for liquid nitrogen is reported in the range of 95 $\mathrm{K}$ to $105 \mathrm{~K}$ [47]. For temperature lower than MFBT, the heat transfer rate increases due to an increase in liquid-solid contacts, and the boiling regime changes from film to the transition boiling regime. In transition boiling, re- 
gions of liquid-solid (nucleate boiling mode) and vapor-solid (film boiling mode) contact occur alternatively at a given location on the heated surface. At the end of the transition boiling regime, the heat flux reaches a maximum value called the critical heat flux (CHF). In the nucleate boiling regime, the maximum amount of liquid-solid contacts occurs. The heat flux in this regime decreases as a result of a reduction in the wall super-heat $\left(\Delta T=T_{\text {wall }}-T_{\text {liquid }}\right)$ values.

As discussed before, the cryo-vials used for the tissue snap-freezing are made of polypropylene or aluminum. The thermal properties of these materials are very different, resulting in different boiling regimes during quenching in liquid nitrogen. The three boiling regimes discussed above are only peculiar to the materials with high thermal effusivity, whereas materials with low thermal effusivity show very high values of heat flux at the liquid-solid interface. The thermal effusivity $(\varepsilon=\sqrt{\lambda \rho c})$ of a material is a measure of the material's ability to exchange thermal energy with its surroundings. It is important to the reader to realize that the thermal effusivity $(\varepsilon=\sqrt{\lambda \rho c})$ should not be confused with the thermal diffusivity ( $\alpha=\frac{\lambda}{\rho c}$ ), while the two expressions contain the same parameters, they are quite different. Thermal diffusivity is related to the speed at which thermal equilibrium can be reached when two bodies touch each other, whereas thermal effusivity (sometimes called the heat penetration coefficient) is the rate at which a material can absorb heat. In contrast to thermal diffusivity, thermal effusivity is proportional to both heat capacity and thermal conductivity of the material, the factors that contribute to materials ability to store and propagate heat, which in turn determines the amount of heat a material can exchange with its surroundings. Thermal effusivity is the property that determines the contact temperature of two bodies that touches each other. If two previously separated infinitely long materials of different effusivities $\left(\varepsilon_{1}\right.$ and $\left.\varepsilon_{2}\right)$ and different temperatures $\left(T_{1}\right.$ and $\left.T_{2}\right)$ suddenly come into contact, then the surface of each material at the contact interface between the two will quickly reach a temperature of $T_{\text {interface }}=T_{1}+\left(T_{2}-T_{1}\right)\left[\frac{\varepsilon_{2}}{\varepsilon_{2}+\varepsilon_{1}}\right]$ i.e. the contact interface temperature will be close to the material with high thermal effusivity. This explains the well-known but often misinterpreted effect that why a metal feels cold to the touch and wool warm, even when both are at the same ambient temperature; this is due to the higher thermal effusivity of the metal compared to the wool. Researchers at TU Eindhoven, 
Netherlands [48] performed a study to visualize the impact of thermal diffusivity and effusivity separately in varying thermal surroundings. To do this, the same temperature boundary condition is imposed on materials with the same thermal effusivity but different thermal diffusivity and vice versa. The results from their study are shown below in Table 2.1. It can be seen that the surface heat flux is proportional to the thermal effusivity value, whereas thermal penetration depth is proportional to the thermal diffusivity value.

$\begin{array}{lllll} & \alpha & \varepsilon & d^{*} & q \\ & \left(\mathrm{~m}^{2} / \mathrm{s}\right) & \left(\mathrm{Ws}^{1 / 2} \mathrm{~m}^{-2} \mathrm{~K}^{-1}\right) & (\mathrm{cm}) & \left(\mathrm{Wm}^{-2}\right) \\ \text { Rockwool } & 3 \times 10^{-6} & 22 & 31.6 & 22.8 \\ \text { Sandstone } & 3 \times 10^{-6} & 3005 & 31.6 & 384.4 \\ \text { Asphalt } & 6.5 \times 10^{-8} & 785 & 4.7 & 100.4 \\ \text { Gypsum } & 1 \times 10^{-6} & 785 & 18.2 & 100.4\end{array}$

Table (2.1) Calculated values of thermal penetration depth $\left(d^{*}\right)$ where temperature amplitude reduced to $33 \%$ and surface heat flux $(q)$ for different materials imposed to fluctuating temperature boundary conditions [48].

Several researchers [39-44, 49-51] have reported on obtaining a higher heat flux by coating the metals using materials with low thermal effusivity. The higher heat flux is obtained by an early transition to the nucleate boiling regime suspected due to a large temperature drop at the coating surface in contact with the liquid. We also observed similar behavior during cool-down of the polypropylene cryo-vial in liquid nitrogen, as the thermal effusivity of polypropylene $\left(\varepsilon_{p p}=564 \mathrm{Ws}^{1 / 2} \mathrm{~m}^{-2} \mathrm{~K}^{-1}\right)$ is very low compare to metals such as aluminum and copper $\left(\varepsilon_{\text {metals }} \approx 10^{4} \mathrm{Ws}^{1 / 2} \mathrm{~m}^{-2} \mathrm{~K}^{-1}\right)$. In this observation, we captured the boiling phenomenon around the aluminum and polypropylene vials using a high-speed camera at the start of the quenching process in liquid nitrogen and found a significant difference between both as shown in the Figure 2.4. A vapor film can be seen around the aluminum vial, whereas a rigorous boiling phenomenon similar to the nucleate boiling regime is found around the polypropylene vial.

\subsection{Materials and methods}

For bench-marking experiments, commercially available aluminum, polypropylene vials are used. The actual pictures of the vials used, their schematic 
representation, and dimensions are shown in Figure 2.5. Experiments are performed by holding the empty vials from the top in a vertical position and subsequently immersing it into the liquid pool. To reduce the parasitic heat load, the containers are held using a holder made of a low conductive thin plastic tube. Before each experiment, the isopentane and ethanol pools are stirred well using a magnetic stirrer to eliminate temperature gradients inside the liquid.

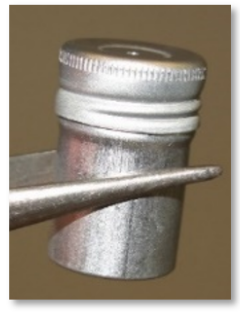

Aluminum vial

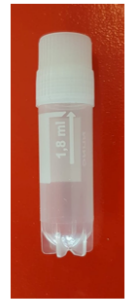

Polypropylene vial

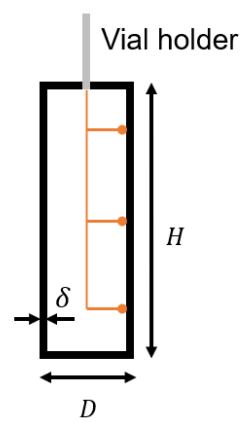

Type-E thermocouple

\begin{tabular}{l|l|l|} 
& Aluminum vial & $\begin{array}{l}\text { Polypropylene } \\
\text { vial }\end{array}$ \\
\hline Weight, W $(\mathrm{g})$ & 1.40 & 2.0 \\
\hline Wall thickness, $\delta(\mathrm{mm})$ & 0.20 & 1.5 \\
\hline Height, H $(\mathrm{mm})$ & 24.50 & 40 \\
\hline Diameter, D $(\mathrm{mm})$ & 14.50 & 12 \\
\hline
\end{tabular}

Stainless steel tube

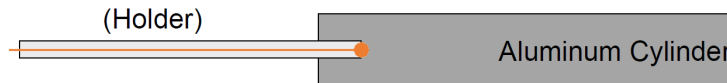

Figure (2.5) Pictures of the cryo-vials and aluminum cylinder used and their schematic representation.

Three thermocouples are attached at various heights inside the vial using aluminum tape to measure the local temperature of the inner wall. From our measurements, it is found that all three thermocouples read out approximately the same temperature variation, which is due to the fact that the thickness of 
the vial-wall is small compared to its length i.e., one-dimensional heat transfer is expected for the polypropylene vial-wall whereas the aluminum vial can be assumed as a lumped capacitance; this will be discussed later in detail. The same temperature reading for all the sensors attached to the inner wall, therefore, ensures good thermal contact of the thermocouple with the inner-wall. To demonstrate the repeatability, three independent experimental runs were performed for each measurement. Measurements are recorded at a speed of 100 samples per second. As the cooling speed of the vials is approximately $20 \mathrm{~K} / \mathrm{s}$ with the measurement speed used, thus a resolution of approximately $0.2 \mathrm{~K}$ is obtained, which is good enough to capture the transient cooling characteristics of the containers.

\subsection{Experimental results}

The temperature-time measurements of both vials being quenched in different coolants are shown in Figure 2.6. The cooling of the aluminum vial in liquid nitrogen is visibly slower than in isopentane or ethanol, whereas polypropylene vial cools faster in liquid nitrogen. The reason for a polypropylene vial to cool faster is due to the earlier transition from film to nucleate boiling regime, as discussed before. This leads to a higher heat transfer rate at the liquidsolid interface of the polypropylene vial compared to the aluminum vial, for which the heat transfer is mainly occurring in the film boiling regime. On the other hand, the heat transfer in isopentane and ethanol is driven by natural convection, which has fairly the same order of heat transfer coefficient for aluminum and polypropylene vials. However, due to the poor thermal diffusivity of the polypropylene, the polypropylene vial cools slower compared to the aluminum vial in isopentane and ethanol. 


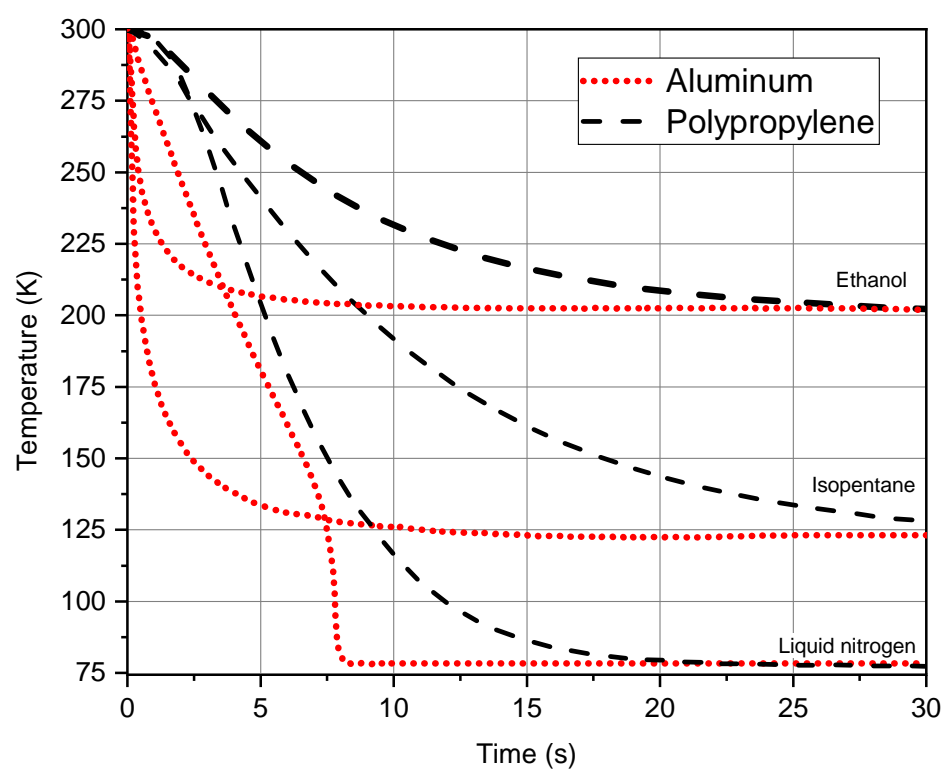

Figure (2.6) Measured cool-down of the cryo-vials in different coolants. The temperature is measured using type-E thermocouples with an accuracy of \pm 2 $\mathrm{K}$, which are fixed onto the inner side wall of the cryo-vial.

\subsection{Heat transfer in isopentane and ethanol pool}

The calculated heat transfer coefficient values obtained using temperaturetime measurements for the aluminum vial are shown in Figure 2.7. To calculate this, the aluminum vial is assumed as a lumped mass, which is a valid assumption as the Biot number $(B i)$ - indicating the ratio of thermal resistance within the vial compared to that between the vial and the coolant, $B i=\frac{h \delta}{\lambda_{A l}}<$ 0.1 (typically $h \approx 10^{2} \mathrm{Wm}^{-1} \mathrm{~K}^{-1}$ ) and the Fourier number $(F o)$ - indicating the heat diffusion time inside the vial wall, $F o=\frac{\alpha t}{\delta^{2}}=1$, for $t \approx 4 \mathrm{~ms}$. The values of Biot and Fourier numbers are calculated correspond to the worst case scenario, in which thermal properties values at room temperature is considered. For aluminum, thermal conductivity increases, while heat capacity decreases with the decrease in temperature (see Figure 2.1). Using the lumped mass assumption, the heat transfer coefficient values are estimated using, 


$$
h=\frac{-m c_{\mathrm{Al}} \frac{d T}{d t}}{\left(T-T_{\infty}\right)}
$$

In this, $m$ and $c_{\mathrm{Al}}$ are the mass and specific heat capacity of the aluminum vial, respectively, $h$ is the heat transfer coefficient between the vial and the coolant, and $T_{\infty}$ is the coolant bath temperature. The values of the time derivative of the temperature variation of $\frac{d T}{d t}$ are obtained from the temperature-time measurements.

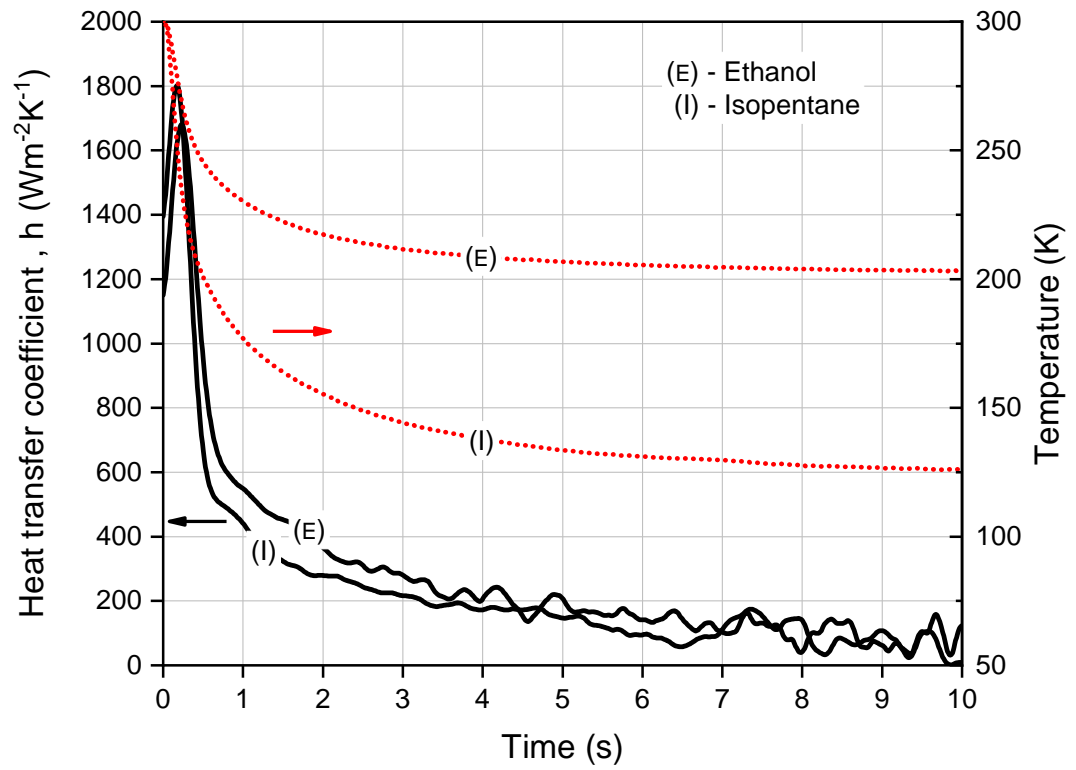

Figure (2.7) Heat transfer coefficient $(h)$ of aluminum vial calculated from the temperature measurements using Equation 2.1.

It can be seen that the heat transfer coefficient values during the immersion process ( $\mathrm{t}<1$ second) is one order of magnitude higher than after the immersion. During the immersion, the aluminum vial cools from around $295 \mathrm{~K}$ to approximately $180 \mathrm{~K}$ and $230 \mathrm{~K}$ for isopentane and ethanol bath, respectively, in around $1 \mathrm{~s}$. This is caused by the increased fluid flow around the vial during the immersion of the vial. The fast thermal response of the aluminum vial to these increased heat transfer coefficient values is due to its low heat capacity 
$(1.4 \mathrm{~J} / \mathrm{K})$ and high thermal diffusivity, which allows it to interact faster with its surrounding fluid.

Next, it is shown that if a larger heat capacity is immersed in a controlled manner into a sub-cooled ethanol bath, the increased cool-down observed during the immersion process can be suppressed and, therefore, for a well-defined geometry, its cool-down can be predicted using the correlations available for the heat transfer due to the natural convection $[26,27]$. To do this, an aluminum cylinder with a heat capacity three times larger than the aluminum vial and with dimensions of $60 \mathrm{~mm}$ length and $6 \mathrm{~mm}$ diameter is immersed with the cylinder axis positioned horizontally into an ethanol bath pre-cooled to approximately $200 \mathrm{~K}$. After the immersion, the cylinder is held stationary using a thin stainless steel tube of $1.2 \mathrm{~mm}$ outer diameter and $0.1 \mathrm{~mm}$ wall thickness glued to the side face of the cylinder (see Figure 2.5). The length of the stainless steel tube inside the liquid pool is approximately $10 \mathrm{~cm}$ for each experiment. Due to the low heat capacity of the tube, it cools faster compared to the cylinder and, therefore, the parasitic heat load through the tube can be neglected in the present analysis. The large length of the tube inside the liquid pool also helps in further reducing the relative contribution of the parasitic heat load through the tube. The measurements are recorded at a rate of 100 samples per second. The temperature of the aluminum cylinder is measured at its center using a type-E thermocouple. The temperature variation of the cylinder during its cool-down is shown in Figure 2.8a. A single point temperature measurement represents the temperature of the entire cylinder, as the cylinder can be assumed as a lumped capacitance. This assumption is valid as the Biot number, $\mathrm{Bi}<0.1$ and the Fourier number indicating the heat diffusion time in the cylinder, $\mathrm{Fo}=\frac{4 \alpha \mathrm{t}}{\mathrm{D}^{2}}=1$, for $\mathrm{t}=0.09 \mathrm{~s}$. Using this, the heat transfer coefficient during the cool-down of the aluminum cylinder is calculated using Equation 2.1 and is plotted against time in Figure 2.8b. Similar to the aluminum vial, an increased heat transfer coefficient is observed during the immersion time ( $\mathrm{t}<1$ second). However, in this case, due to a higher heat capacity, the temperature of the cylinder does not drop significantly. To predict the cool-down of the cylinder, a numerical model built in COMSOL is used. Here, a limited heat transfer coefficient due to the natural convection from an isothermal horizontal cylinder is assumed at the liquid-solid boundary, which is given by [45], 


$$
h=\frac{\lambda}{D}\left(0.6+\frac{0.387 R a_{D}{ }^{1 / 6}}{\left(1+\left(\frac{0.559}{P r}\right)^{9 / 16}\right)^{8 / 27}}\right)^{2}
$$

in this, $P r$ is the Prandtl number and $R a_{D}$ is average Rayleigh number calculated based on cylinder diameter. Equation 2.2 is valid for $10^{-5}<R a_{D}<10^{12}$ and the entire Prandtl number. The numerical model is solved to estimate the temporal temperature variation of the cylinder, and a solution independent of the grid size is presented here. The estimated temperature values agree fairly well with the temperature measurements within the measuring accuracy of $\pm 2 \mathrm{~K}$, as shown in the Figure 2.8a.

The increased heat transfer coefficient at the vial-liquid interface during the immersion process is not confirmed for the polypropylene vials, as the temperature of the vial is measured at its inner wall and due to low thermal diffusivity a temperature gradient exists across the vial-wall. However, one can expect similar effects for the polypropylene vial also, as the increased heat transfer is caused due to the increased fluid flow around the vial is caused during its immersion into the liquid, which is independent of the material thermal properties.

The heat transfer coefficient in the case of natural convection is a function of several parameters that include the object dimensions, shape, and orientation [26, 27]. The vials used for the snap-freezing are not standardized and have different shapes. Also, an uncontrolled manual immersion of the vial results in numerous orientations; this non-standardized quenching process results in heat transfer coefficient values that can not be predicted. Therefore, developing a generalized heat transfer model for cryo-vials in isopentane and ethanol is not feasible and is not pursued in this work. 


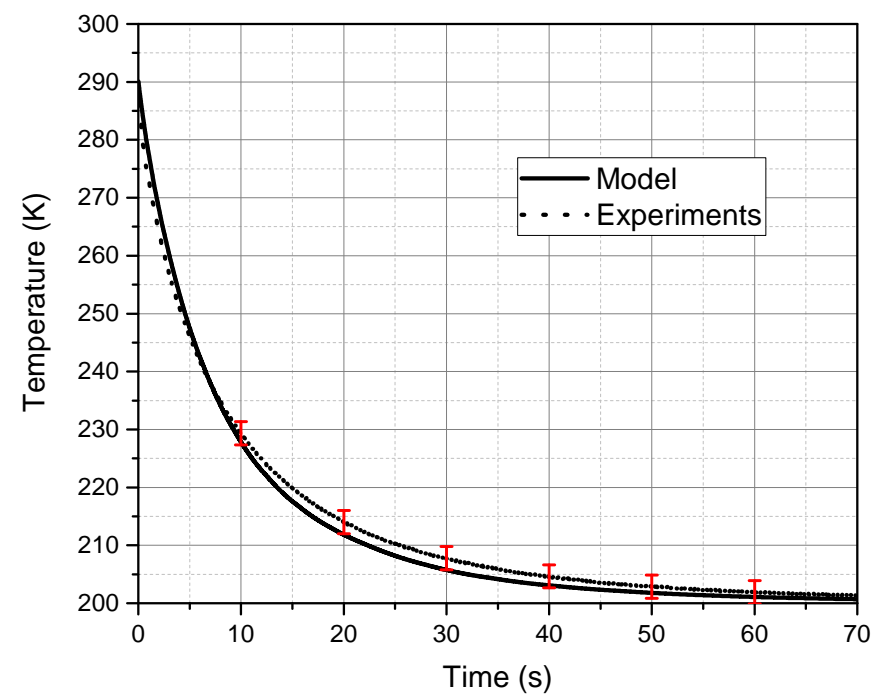

(a)

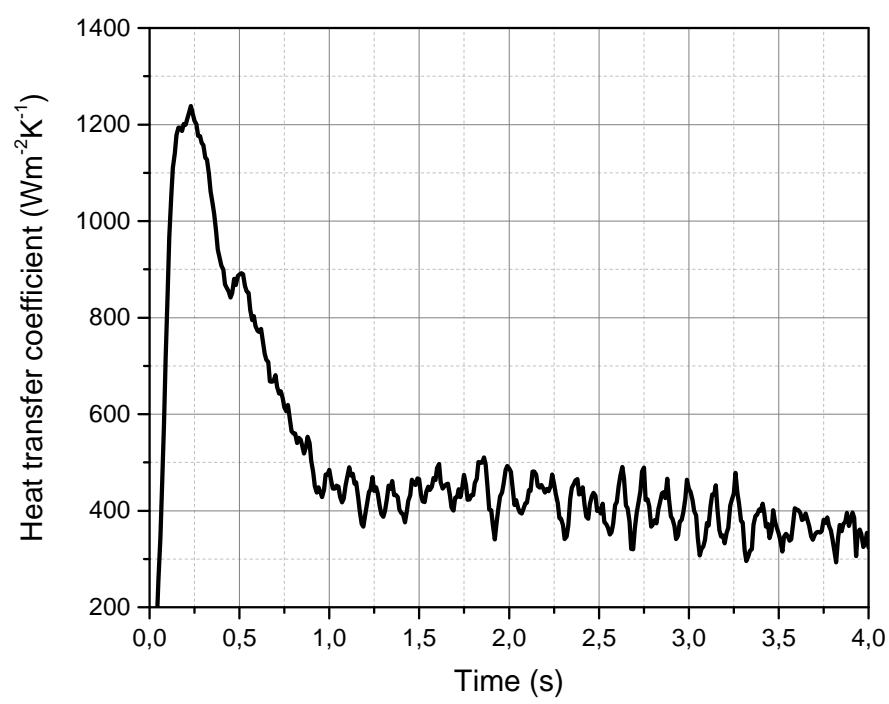

(b)

Figure (2.8) Aluminum cylinder in per-cooled ethanol bath (a) Comparing temperature measurements with the estimated values using numerical model (b) Calculated $h$ values from the temperature measurements using Equation 2.1 


\subsection{Heat transfer in liquid nitrogen}

As discussed before in section 2.1, boiling heat transfer in liquid nitrogen is strongly dependent on the material thermal properties. Therefore the heat transfer phenomenon for the aluminum and the polypropylene material is discussed separately.

Aluminum vial: For materials with high thermal effusivity like aluminum, heat transfer occurs mainly in the film boiling regime, for which the heat transfer coefficient is a function of many parameters also including the object dimensions, shape, and orientation. Bromley [21] first predicted the film boiling heat transfer coefficient for a horizontal cylinder. However, the study did not include the effect of the cylinder curvature. This effect was later included in the study reported by Breen [22]. Wafer [25] reported the effect of the diameter on the film boiling behavior in liquid nitrogen. Beduz et al. [28] reported the angular dependence of boiling heat transfer mechanisms in liquid nitrogen. Predicting cooling of the vial in liquid nitrogen requires accurate prediction of the heat transfer coefficient $(h)$; however, the dependence of $h$ values on so many parameters makes it infeasible to develop a generalized heat transfer model that accounts different shape, dimensions and orientation of the vial immersed and, therefore, is not discussed in this work.

Polypropylene vial: As discussed before in section 2.1, several researchers [39-44, 49-51] have reported obtaining higher heat flux by coating the metals using materials with low thermal effusivity. The higher heat flux is obtained by an early transition to the nucleate boiling regime suspected due to a large temperature drop at the coating surface in contact with the liquid. The maximum cooling rate can be obtained using an optimum coating thickness value. For a coating thickness higher than the optimum value, cooling time increases due to the increased thermal resistance offered by the coating material. This suggests that the cooling speed during quenching of the materials with low thermal effusivity is largely limited by the material itself and not by the liquid. Keeping this mind, we have developed a heat transfer model in which thermal resistance to heat transfer at the liquid-solid interface is neglected compared to the thermal resistance inside the polypropylene material and a Perfect Thermal Contact Assumption is assumed at the liquid-solid interface. A fairly good prediction of the temperature variation at the inner wall of an 
empty polypropylene tube is obtained with a maximum error of less than $10 \%$ compared to measurements. The cool-down time of the inner wall of an empty tube scales with the square of its wall thickness. The details of the models are discussed in the next chapter.

\subsection{Conclusions}

Polypropylene vial cools faster in saturated liquid nitrogen than in sub-cooled isopentane and ethanol, whereas the opposite is true for the case of the aluminum vial. The reason for the polypropylene vial to cool faster is due to the early transition to the nucleate boiling regime. On the other hand, in the case of sub-cooled liquids, the heat transfer is due to the natural convection. A higher heat transfer rate caused due to the forced flow is achieved during the immersion of vials into the coolant, which results in faster cool-down of the aluminum vial. This is due to its higher thermal diffusivity, which allows it to interact faster with its surrounding fluid whereas, no such effect is observed in the case of the polypropylene material, which has relatively lower thermal diffusivity. 
CHAPTER 3

\title{
How a polypropylene vial cools in liquid nitrogen? ${ }^{1}$
}

\begin{abstract}
Snap-freezing of a tissue is usually realized by enclosing it in a polypropylene cryo-vial and subsequently immersing the vial in liquid nitrogen. The cooling rate of the vial is very critical to the frozen-tissue quality. However, quantitative information on the heat transfer process at the vial-liquid interface is still a mystery limiting the cool-down prediction of the vial and the tissue. In this chapter, an analytical model is developed to predict the temperature variation inside the polypropylene vial-wall and the tissue. This model also include temperature-dependent thermal properties of the polypropylene material. The cooling time of an empty polypropylene tube of wall thickness $1.5 \mathrm{~mm}$, replicating a typical commercial polypropylene vial is predicted with a maximum error of less than $10 \%$. We also verified the model using temperature-time measurements performed with several other polypropylene tubes of different wall thickness. An interesting finding from this work is that the difference in the cool-down time of a tissue in a tube and an empty tube is proportional to the product of the tissue heat capacity and the tube-wall thermal resistance. This enables researchers to estimate the cooling trajectory of the tissue during the cooling process allowing the development of improved snap-freezing protocols.
\end{abstract}

\subsection{Introduction}

Thermal quenching is a process where a hot object is rapidly cooled by immersing it in coolant and is of great interest to many applications including

\footnotetext{
${ }^{1}$ Published as : Jagga, S. and Vanapalli, S., 2020. Cool-down time of a polypropylene vial quenched in liquid nitrogen. International Communications in Heat and Mass Transfer, 118, p.104821.
} 
cryo-preservation [13]. In cryo-preservation, biological materials such as cells and tissues are physically fixed by snap-freezing. In the snap-freezing process, the material is enclosed in a container called a cryo-vial, which is then rapidly immersed in a liquid nitrogen bath. Commonly used vials are in the shape of tubes made of polypropylene material. Baucamp et. al. [14] showed that the quality of the frozen-tissue depends strongly on its cooling rate. Since the vial wall acts as a thermal interface between the liquid and the bio-material, quantitative prediction of the temperature in the vial-wall is critical to the tissue temperature prediction. The temperature variation inside the vial-wall can only be determined for the known values of the heat transfer rate at the wallliquid interface, which is not known. This limits the cool-down prediction of the cryo-vial wall and therefore of the tissue attached to it. The heat transfer rate at the vial-liquid interface is due to the boiling phenomenon and therefore depends upon several parameters that also include the vial shape and dimensions. These parameters for the vial are not standardized largely due to the different tissue sample size and vial manufacturers. In this paper, we present a heat transfer model that predicts the temperature variation of the vial-wall and to do so it requires only wall-thickness as an input parameter. This exempts us to consider shape and other dimensional parameters such as height and diameter for estimating the heat transfer rate at the vial-wall.

A brief introduction to the boiling phenomenon in saturated liquid nitrogen is presented here to help in understanding the heat transfer aspects of the snap-freezing process. Three different modes, namely film, transition and nucleate boiling [46] occur depending on the wall super-heat temperature $\left(\Delta T_{\text {wall }}=T_{\text {wall }}-T_{\text {liquid }}\right)$. At a high wall super-heat, the vapor generated forms a layer between the wall and the liquid limiting the heat transfer to the liquid. The thickness of the vapor layer reduces with the reduction in the wall superheat and collapses when the wall temperature drops below the minimum film boiling temperature. After this, a transition to the nucleate boiling regime occurs where because of increased liquid-solid contacts higher heat flux is obtained. These three regimes are only peculiar to the materials with high thermal effusivity, while material with low thermal effusivity shows overall different cooling behaviour [40]. The thermal effusivity $(\varepsilon=\sqrt{\lambda \rho c})$ of a material is a measure of the material's ability to exchange thermal energy with its surroundings. Several researchers [39-44, 49-51] have reported obtaining 
higher heat flux by coating the metals using materials with low thermal effusivity. The higher heat flux is obtained by an early transition to the nucleate boiling regime suspected due to a large temperature drop at the coating surface in contact with the liquid. The maximum cooling rate can be obtained using an optimum coating thickness value. For coating thickness higher than the optimum value, cooling time increases due to the increased thermal resistance offered by the coating material. This suggests that cooling speed during quenching of the materials with low thermal effusivity is largely limited by the material itself and not the liquid.

We will now discuss previous attempts made to estimate the temperature variation of the materials with a thermal effusivity value of at least one order lower compared to the metals $\left(\varepsilon \sim 10^{4} \mathrm{Ws}^{1 / 2} \mathrm{~m}^{-2} \mathrm{~K}^{-1}\right)$. Concrete has a thermal effusivity value of approximately $1890 \mathrm{Ws}^{1 / 2} \mathrm{~m}^{-2} \mathrm{~K}^{-1}$ and is a material of interest to many researchers for estimating the evaporation rate in case of liquid nitrogen spillage on the concrete floor. To do this, the heat transfer rate at the liquid-solid surface is required, which can be determined for the known temperature variation inside the concrete material during its cool-down. To predict the temperature variation inside the concrete block cooled using liquid nitrogen, Olewski et al. [52,53] assumed that the surface of a concrete block is equal to the liquid nitrogen temperature. This assumption in the literature is named as Perfect Thermal Contact Assumption (PTCA). The calculated temperature variation inside the block did not match well with the measurements. Similarly, Cha et al. [54] and Huang et al. [55] found a significant temperature difference between the concrete block surface and the liquid nitrogen bath especially at the initial phase of the cooling process. In their studies, the initial cooling phase is in the range of 5-7 minutes. In conclusion, the studies reported in the literature have shown that the PTCA assumption is incorrect in estimating the cool-down of the concrete material.

The thermal effusivity of polypropylene $\left(\varepsilon_{\mathrm{pp}}=564 \mathrm{Ws}^{1 / 2} \mathrm{~m}^{-2} \mathrm{~K}^{-1}\right)$ is much lower than that of concrete $\left(\varepsilon_{\text {concrete }}=1890 \mathrm{Ws}^{1 / 2} \mathrm{~m}^{-2} \mathrm{~K}^{-1}\right)$, which suggests a higher temperature drop at the liquid-solid interface of the polypropylene material compared to that in concrete. How the thermal effusivity of the material can affect the interface temperature is illustrated by considering two semiinfinite surfaces of different materials and initial temperatures that are brought in contact. One of the surfaces is assumed to have the same initial temperature 
and thermal properties as saturated liquid nitrogen and the other surface is a fictitious material with an initial temperature of $290 \mathrm{~K}$ and different values of thermal effusivity ranging from 40 to $10^{5} \mathrm{Ws}^{1 / 2} \mathrm{~m}^{-2} \mathrm{~K}^{-1}$. The temperature at the interface is calculated using Equation 3.1 [56] and is shown in Figure 3.1. The interface temperature approaches that of liquid nitrogen for lower values of material thermal effusivity. The results plotted in Figure 3.1 shows that the polypropylene material will experience a significantly higher temperature drop at the liquid-solid interface compared to the concrete material.

$$
T_{\text {int }}=T_{\text {liquid }}+\left(T_{\text {solid }}-T_{\text {liquid }}\right) \frac{\varepsilon_{\text {solid }}}{\varepsilon_{\text {solid }}+\varepsilon_{\text {liquid }}}
$$

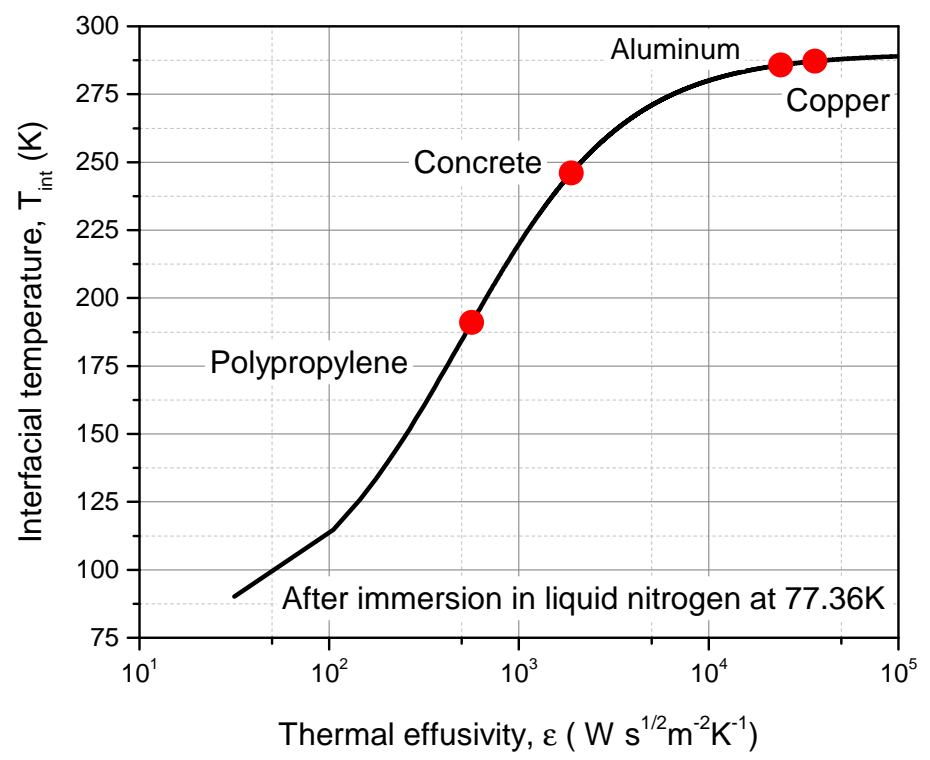

Figure (3.1) Temperature at the interface between two semi-infinite mediums. Here, one of the medium has properties of liquid nitrogen and an initial temperature equal to $77.36 \mathrm{~K}$ and the properties of the second medium are varied. The second medium's initial temperature is $290 \mathrm{~K}$.

The studies [34-36] reported so far to estimate the cool-down of the polypropylene materials are empirical and do not assume temperature-dependent heat 
capacity for the polypropylene material [32]. In the present work, the Perfect Thermal Contact Assumption (PTCA) is re-assessed for the polypropylene material. The advantage of doing this is that it would exempt us from using the correlations that requires the vial shape and dimensions for estimating the heat transfer rate at the vial-liquid interface. To validate this assumption, firstly, a numerical model which also considers temperature-dependent thermal properties for the polypropylene material is developed using a commercial finite element solver. After validating the PTCA using the numerical model, an approximate analytical model considering the one-dimensional heat transfer in the material is also developed to predict the temperature variation inside the material domain and of a sample tissue placed at its inner wall. The analytical model also considers temperature dependence of the thermal properties and is validated using the measurements performed with empty polypropylene tubes of different wall thicknesses.

\subsection{Experimental setup}

Four polypropylene tubes of wall thickness 1.5, 2.0, 2.5, and $3.0 \mathrm{~mm}$ were used in the experiments. The tubes are made by machining inside a polypropylene rod of an external diameter of $20 \mathrm{~mm}$. The tubes are subsequently sealed on both ends with end caps that have the same thickness as the respective tube walls. Three type-E thermocouples are placed along the inner wall of each container. Each thermocouple is attached to the wall using a tiny piece of aluminum tape. To demonstrate the repeatability, three independent experimental runs were performed for each measurement. Figure 3.2 shows a schematic of the containers and Table 3.1 shows the thermal properties of the polypropylene material. Thermal conductivity, $\lambda$ of the polypropylene is fairly constant in the temperature range of $77 \mathrm{~K}$ to $300 \mathrm{~K}$ [33] whereas its heat capacity decreases with temperature $[32,33]$. The thermal diffusivity values for the temperature range of $77 \mathrm{~K}$ to $300 \mathrm{~K}$ are shown in Figure 3.3.

Table (3.1) Thermal properties of polypropylene material

\begin{tabular}{|l|l|}
\hline Thermal conductivity [33], $\lambda\left(\mathrm{Wm}^{-1} \mathrm{~K}^{-1}\right)$ & 0.17 \\
\hline Density, $\rho\left(\mathrm{kgm}^{-3}\right)$ & 905 \\
\hline Heat capacity [32], $c(T)\left(\mathrm{Jkg}^{-1} \mathrm{~K}^{-1}\right)$ & $0.0002297 T^{3}-0.1153 T^{2}+$ \\
& $23.05 T-671.9$ \\
\hline
\end{tabular}




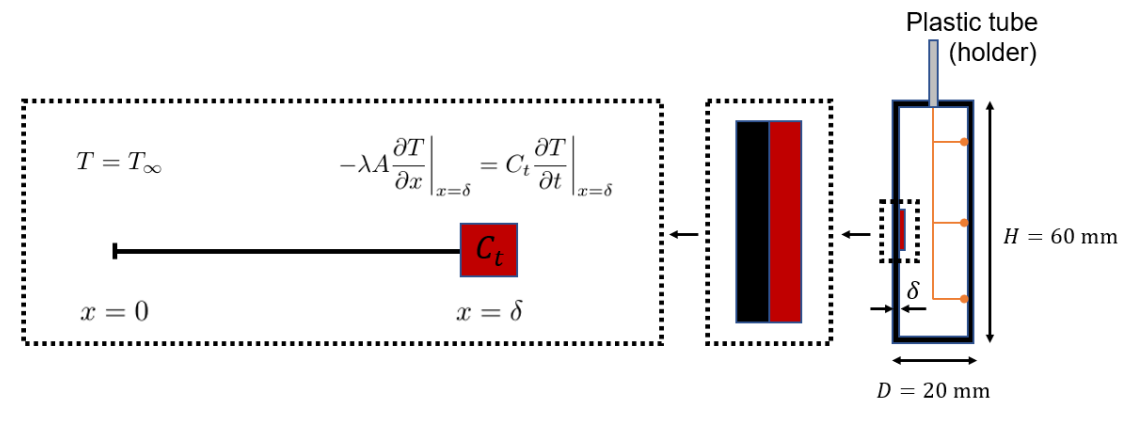

Figure (3.2) Schematic of a polypropylene tube used in the measurements $(\delta=1.5,2.0,2.5$ and $3.0 \mathrm{~mm})$. The description of the model for the tube wall along with the boundary conditions is shown to the left.

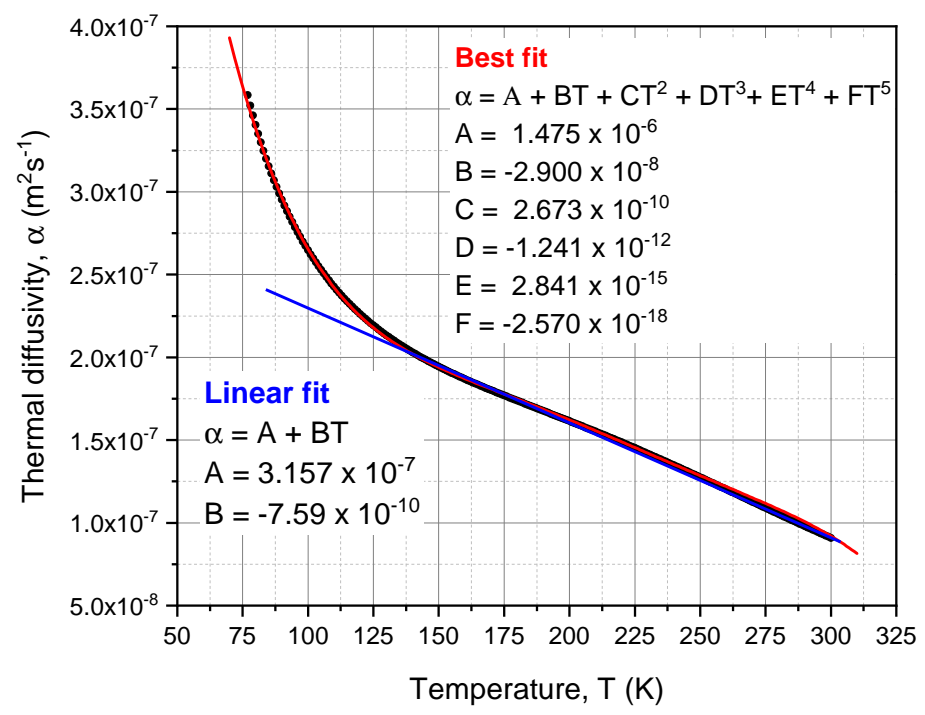

Figure (3.3) Thermal diffusivity, $\alpha=\frac{\lambda}{\rho c}$, of the polypropylene calculated using thermal conductivity $(\lambda)$ [33], density $(\rho)$ and specific heat capacity(c) $[32,33]$. The best interpolation for thermal diffusivity values is obtained using a fifth order polynomial fit in Matlab curve fitting application, however there is no specific reason for choosing a polynomial fit. Linear interpolation is done for the temperature range of $300 \mathrm{~K}$ to $130 \mathrm{~K}$, which is then extrapolated till $77 \mathrm{~K}$. 
Experiments are performed by holding the container from top in a vertical position and subsequently immersing into the liquid nitrogen pool. To reduce the parasitic heat load, the containers are held using a holder made up of a low conductive thin plastic tube. Measurements are recorded at a speed of 100 samples per second, which is fast enough to capture the cooling transient characteristics of the containers.

\subsection{Heat transfer model and experimental validation}

In this section, a heat transfer model to predict the cool-down of the polypropylene tube is developed and the results are compared with experimental data. First, the Perfect Thermal Contact Assumption for polypropylene tube wall is validated by comparing the experimental data with a numerical model. Next, an approximate analytical thermal model is developed considering a linear dependence of thermal diffusivity with temperature. The results from the analytical model are also validated using experimental data. Lastly, using the approximate model, cool down predictions of a lumped heat capacity attached to the tube wall mimicking a tissue is discussed.

A one-dimensional heat transfer model for the tube wall is valid because of a large aspect ratio $\left(\frac{H}{\delta} \approx 20\right)$. The thermal diffusion time in the lateral direction $\left(t_{\delta} \propto \delta^{2}\right)$ is much smaller compared to the longitudinal direction $\left(t_{H} \propto H^{2}\right)$. Also, the heat transfer boundary conditions for most of the surface area at the inner and outer wall of the tube does not change along the y-direction, which suggests negligible temperature gradients in the longitudinal direction of the tube wall.

Instead of a tube geometry, the problem can be described by a slab in this particular case as the ratio of the wall thickness to the diameter $(\delta / D<$ $0.15)$ is small. To substantiate this argument, the thermal resistance of a tube $\left(R_{\text {tube }}=\ln \left(\frac{D}{D-2 \delta}\right) /(2 \pi \lambda H)\right)$ and a slab $\left(R_{\text {slab }}=\delta /(\lambda \tilde{A}) ; \tilde{A}=\pi(D-\delta / 2) H\right)$ as a function of wall thickness is shown in Figure 3.4. In the region of the wall thickness of interest shown as a shaded region in Figure 3.4, the difference in the thermal resistance values is practically negligible. 
The governing equation for the heat transfer along the wall is,

$$
[h !] \frac{\partial^{2} T}{\partial x^{2}}=\frac{1}{\alpha(T)} \frac{\partial T}{\partial t}, \quad \text { in } \quad 0 \leq x \leq \delta, \quad t \geq 0
$$

in this, $T$ is the temperature, $t$ is the time and $\alpha$ is the thermal diffusivity. The initial and boundary conditions are $T(x, t=0)=T_{i} ; T(x=0, t)=$ $T_{\infty} ;\left.\frac{\partial T}{\partial x}\right|_{x=\delta}=0$. An exact solution for predicting the cool-down of the polypropylene material inside the liquid nitrogen pool is not possible due to the temperaturedependent thermal diffusivity of the polypropylene material, which is a fifthorder polynomial function of the temperature (see Figure 3.3). In order to validate the Perfect Thermal Contact Assumption (PTCA), a numerical model is developed which is discussed in the next section.

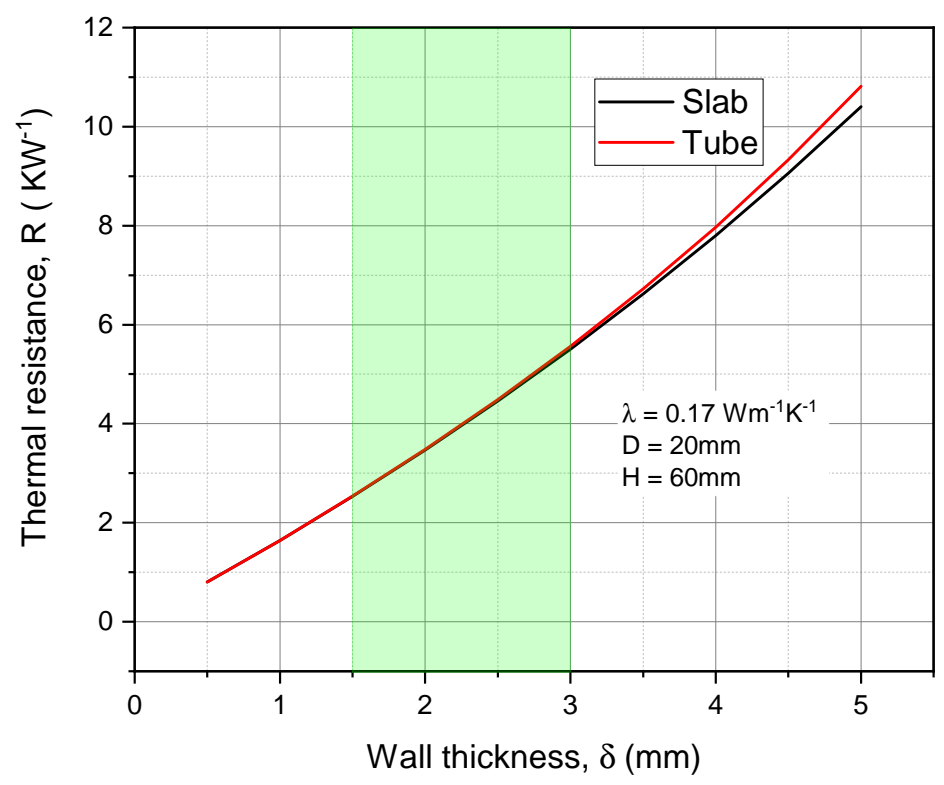

Figure (3.4) Thermal resistance of the polypropylene tube and a slab of different wall thicknesses. The diameter and the height of the tube used for the calculations are $20 \mathrm{~mm}$ and $60 \mathrm{~mm}$. The highlighted area shows the range of the wall thickness used in the experiments. 


\subsubsection{Verification of PTCA}

A one-dimensional model of the polypropylene tube with the boundary conditions defined above is developed in COMSOL. The results of this model are verified by comparing with an analytical solution for a constant thermal diffusivity value given by Equation 3.3,. The analytical solution is derived in the book written by Ozisik [57] and is shown here for completeness,

$$
T^{*}(x, t)=\frac{T(x, t)-T_{\infty}}{T_{i}-T_{\infty}}=\frac{2}{\pi} \sum_{n=1}^{\infty}\left[\frac{1-(-1)^{n}}{n} \sin \left(\frac{n \pi x}{\delta}\right) e^{\left(-\frac{\alpha n^{2} \pi^{2}}{\delta^{2}} t\right)}\right]
$$

Figure 3.5a shows the numerical and analytical solution of temperature at the inner wall $T(\delta, t)$ for four different wall thickness values. A thermal diffusivity value of $1.62 \times 10^{-7} \mathrm{~m}^{2} \mathrm{~s}^{-1}$ is used for the calculations, which is a temperature averaged value between $300 \mathrm{~K}$ and $77 \mathrm{~K}$. The maximum difference in results is less than $1.2 \%$ showing that the COMSOL model can be used for further validation.

To validate the PTCA assumption for this case, the calculated temperature values from the COMSOL numerical model are compared with the measured data at the inner wall of the polypropylene tube, which is shown in Figure 3.5b. In the COMSOL model temperature-dependent thermal diffusivity values are used. The agreement is good particularly in the temperature range of $295 \mathrm{~K}$ to $125 \mathrm{~K}$. Below $125 \mathrm{~K}$, the difference is large to a maximum of 8 $\%$, which could be because for lower temperature values of the inner wall, the percentage error in the heat flux calculation will be higher as the heat flux is largely proportional to the temperature difference between the inner and outer wall.

\subsubsection{Approximate analytical model}

In this section, an analytic expression to predict the temperature variation in the tube-wall and of the tissue attached to it is derived. An analytical solution is feasible when the thermal diffusivity is linearly dependent on temperature, which is the case in the temperature range between $125 \mathrm{~K}$ and $300 \mathrm{~K}$ (see Figure 3.3). Below $125 \mathrm{~K}$ till $77 \mathrm{~K}$, the heat capacity of polypropylene and the heat flux values are low, thus having less impact on the cooling speed. 


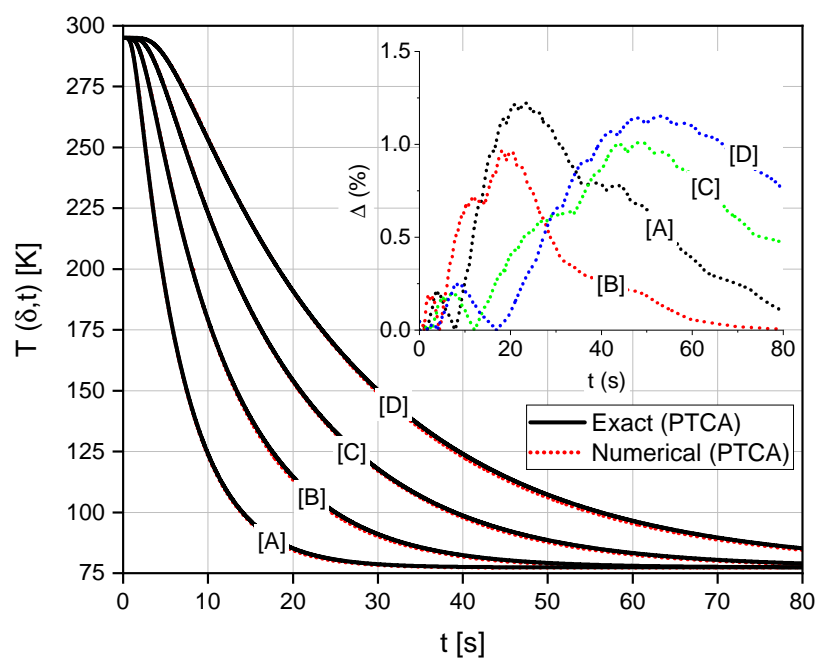

(a)

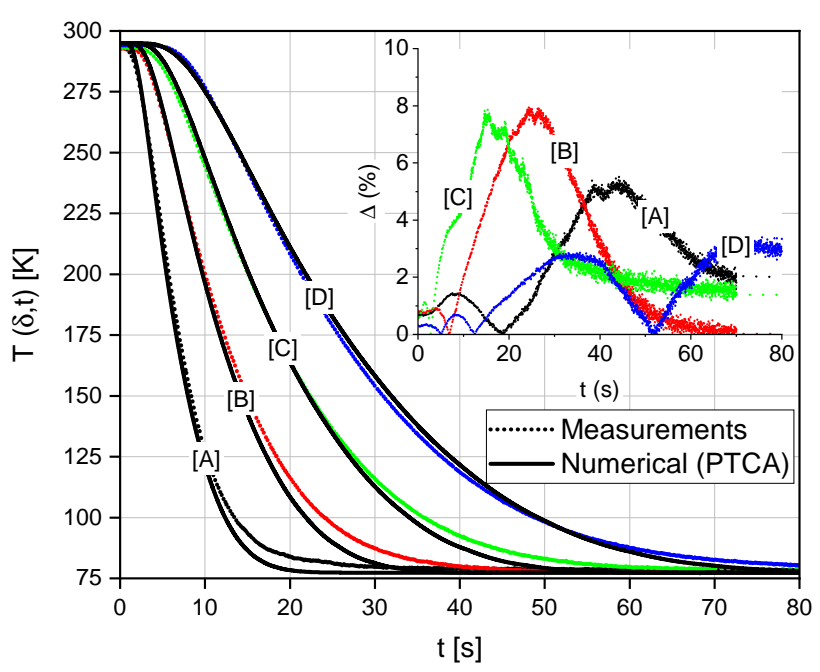

(b)

Figure (3.5) Validating Perfect Thermal Contact Assumption (PTCA) for polypropylene material. The absolute difference between the values compared to the measurements is shown in the inset. Labels [A], [B], [C] and [D] refer to wall thickness value of $1.5 \mathrm{~mm}, 2.0 \mathrm{~mm}, 2.5 \mathrm{~mm}$ and $3.0 \mathrm{~mm}$, respectively. (a) Comparison of numerical and analytical model with a constant thermal diffusivity value. (b) Comparison of numerical results with temperature-dependent thermal diffusivity and measurement AQta. 
Equation 3.2 is solved using an approximate approach. A cubic polynomial of temperature in the spatial dimension, $x$, is assumed inside the tube-wall. The cubic approximation is due to the limited number of initial and boundary conditions as discussed below,

$$
T(x, t)=a+b x+c x^{2}+d x^{3}
$$

The calculations of the coefficients $(a, b, c$, and $d)$ in Equation 3.4 are discussed in two distinct sections, namely early and late regime. The early regime is the time taken for the cooling front to reach the inner wall of the tube, therefore during the early regime there is no temperature change at the inner wall i.e. $T\left(\delta, t \leq t_{\delta}\right)=T_{i}$, in this, $t_{\delta}$ is the time for the early regime. The time associated with the cooling after the early regime is defined as the late regime $\left(t>t_{\delta}\right)$.

\section{Early regime}

To simplify the analysis, the thermal properties of the polypropylene is assumed constant during the early regime. This assumption is justified as the time duration of the early regime is usually very low compared to the total cool down time of the wall. For a cubic temperature profile inside the wall, the time duration for the early regime can be approximated as [57], $t_{\delta} \approx \frac{\delta^{2}}{8 \tilde{\alpha}}$. In this, $\tilde{\alpha}$ is the temperature averaged thermal diffusivity calculated over the temperature range of $295 \mathrm{~K}$ to $77 \mathrm{~K}$. The boundary conditions for the early regime are given by,

$$
\begin{gathered}
T(x=0, t)=T_{\infty} \\
\left.\frac{\partial T}{\partial t}\right|_{x=0}=\left.\frac{\partial^{2} T}{\partial x^{2}}\right|_{x=0}=0
\end{gathered}
$$

The temperature of the lumped mass at the end of the early regime is equal to the initial temperature i.e. $T\left(\delta, t_{\delta}\right)=T_{i}$. Using these boundary conditions, the temperature of the lumped mass at the end of the early regime $\left(t=t_{\delta}\right)$ is given by, 


$$
T\left(\delta, t_{\delta}\right)=T_{i}=T_{\infty}+b\left(t_{\delta}\right) \delta+d\left(t_{\delta}\right) \delta^{3}
$$

in this, $b$ and $d$ are time-dependent variables, expressions for which are derived in the next section.

\section{Late regime}

During the late regime $\left(t>t_{\delta}\right)$, the initial and boundary conditions are given by,

$$
\begin{gathered}
T\left(\delta, t_{\delta}\right)=T_{i} \\
-\left.\lambda A \frac{\partial T}{\partial x}\right|_{x=\delta}=\left.C_{t} \frac{\partial T}{\partial t}\right|_{x=\delta} \\
T(x=0, t)=T_{\infty}
\end{gathered}
$$

this implies,

$$
\left.\frac{\partial T}{\partial t}\right|_{x=0}=\left.\frac{\partial^{2} T}{\partial x^{2}}\right|_{x=0}=0
$$

Using these initial and boundary conditions, the cool-down time for the tissue attached to the inner-wall of the tube is given by (for complete derivation see Appendix),

$t-t_{\delta}=\frac{I_{1} \ln \left(\left|\frac{d}{d_{i}}\right|\right)}{C_{4}}-\frac{\sum_{\left\{Z: C_{9} Z^{3}+C_{8} Z^{2}+C_{7} Z+C_{4}=0\right\}} \frac{\left[\left(I_{1} C_{7}-I_{2} C_{4}\right)+\left(I_{1} C_{8}-I_{3} C_{4}\right) Z+\left(I_{1} C_{9}\right) Z^{2}\right] \ln \left(\left|\frac{d-Z}{d_{i}-Z}\right|\right)}{\left[C_{7}+\left(2 C_{8}\right) Z+\left(3 C_{9}\right) Z^{2}\right]}}{C_{4}}$

in this, $d_{i}=d\left(t_{\delta}\right)$, is calculated by solving the second order polynomial obtained using Equation 3.7 and the value of $b$ from Equation 3.17,

$$
\left[C_{2} \delta-C_{3} \delta^{3}\right] d_{i}^{2}+\left[\delta^{3}+C_{1} \delta+C_{3}\left(T_{i}-T_{\infty}\right)\right] d_{i}-\left[T_{i}-T_{\infty}\right]=0
$$


Here,

$$
\begin{aligned}
& C_{1}=6 \delta A_{t} \alpha\left(T_{\infty}\right)-3 \delta^{2} ; \quad C_{5}=\frac{6}{5} \beta_{1} \delta^{5}+2 \beta_{1} \delta^{3} C_{1}-C_{3} C_{4} ; \quad C_{9}=-C_{3} C_{6} ; \\
& C_{2}=6 \delta^{4} A_{t} \beta_{1} ; \quad C_{6}=2 \beta_{1} \delta^{3} C_{2}-C_{3}\left(\frac{6}{5} \beta_{1} \delta^{5}\right) ; \quad I_{1}=\frac{C_{1} \delta^{2}}{2}+\frac{\delta^{4}}{4} ; \\
& C_{3}=6 \delta^{2} A_{t} \beta_{1} ; \quad C_{7}=C_{5}-C_{3} C_{4} ; \quad I_{2}=C_{2} \delta^{2}-\frac{C_{3} \delta^{4}}{2} ; \\
& C_{4}=3 \delta^{2} \alpha\left(T_{\infty}\right) ; \quad C_{8}=C_{6}-C_{3} C_{5} ; \quad I_{3}=-C_{2} C_{3} \frac{\delta^{2}}{2}+\frac{C_{3}^{2} \delta^{4}}{4} \text {; }
\end{aligned}
$$

To compare the approximate solution with the measurements performed using empty polypropylene tubes, the solution for no lumped mass at the inner end of the tube wall i.e. $C_{t}=0$ is obtained from the above solution, for complete derivation see Appendix. The solution of the temperature profile in the wall of an empty polypropylene tube wall is,

$$
T^{*}\left(x^{*}, t^{*}\right)=\frac{T\left(x^{*}, t^{*}\right)-T_{\infty}}{T_{i}-T_{\infty}}=\frac{\frac{3}{2} \alpha\left(T_{\infty}\right)\left[x^{*}-\frac{x^{* 3}}{3}\right] e^{\frac{-12}{5} \alpha\left(T_{\infty}\right)\left(t^{*}-\frac{1}{8 \alpha(\widetilde{T})}\right)}}{\alpha\left(T_{\infty}\right)+\frac{4}{5} B\left(T_{i}-T_{\infty}\right)\left[1-e^{\frac{-12}{5} \alpha\left(T_{\infty}\right)\left(t^{*}-\frac{1}{8 \alpha(\widetilde{T})}\right)}\right]}
$$

in this, $x^{*}=\frac{x}{\delta}$ and $t^{*}=\frac{t}{\delta^{2}}$. It is interesting to see that the cool-down time at location $x^{*}$ is proportional to the square of the wall thickness, as also observed in the solution obtained for constant thermal properties (see Equation 3.3). The predicted temperature values at the inner wall $\left(x^{*}=1\right)$ using Equation 3.14 are now compared with the measurements in Figure 3.6. The percentage difference between these values compare to the measurements is also shown there. It can be seen that the approximate model is able to predict the cool-down time with a maximum error of less than $10 \%$. The difference between the values reduces significantly for higher wall thickness and is approximately $4 \%$ for the wall thickness value of $3 \mathrm{~mm}$.

In Figure 3.7, the calculated dimensionless temperature $T^{*}$ from Equation 3.14 is compared with the measurement data. According to the approximate model, in the early regime, the inner wall temperature will not be influenced, therefore from the start till $t^{*} \lesssim 0.7 \mathrm{~s} \mathrm{~mm}^{-2}$ the calculated $T^{*}$ values are constant and equal to one. The calculated cooling curves for all the tubes collapse together for the approximate solution, as is also expected from Equa- 


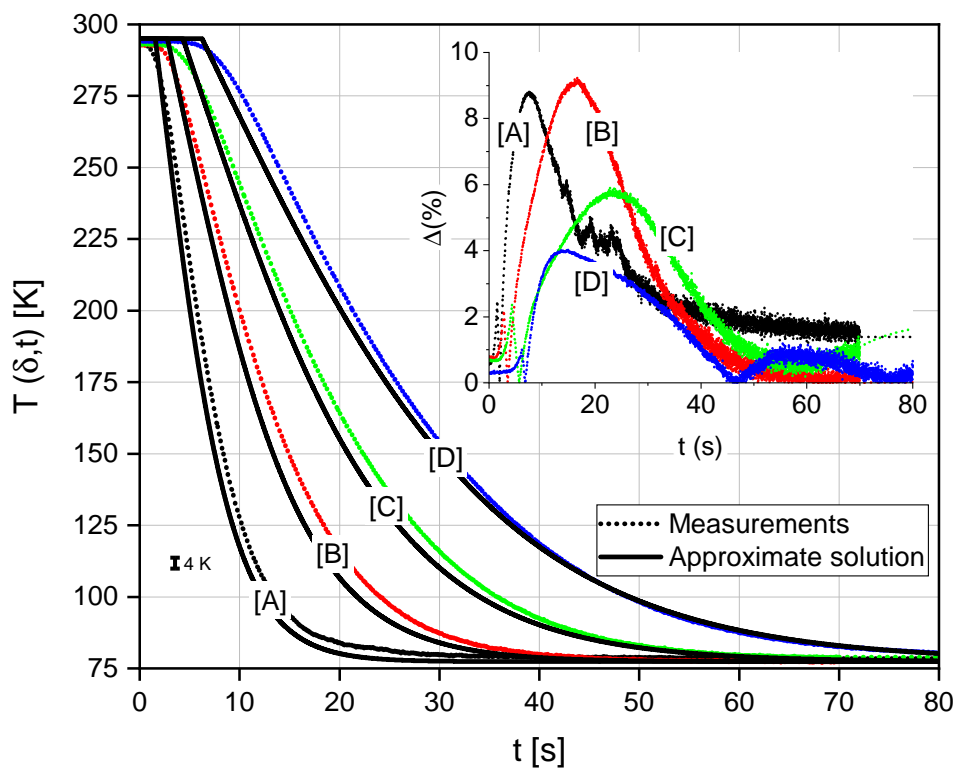

Figure (3.6) Comparison of the temperature variation at the inner wall of the tube. The absolute difference between the values compared to measurements is shown in the inset. Curves $[\mathrm{A}],[\mathrm{B}],[\mathrm{C}]$ and $[\mathrm{D}]$ refer to wall thickness values of $1.5 \mathrm{~mm}, 2.0 \mathrm{~mm}, 2.5 \mathrm{~mm}$ and $3.0 \mathrm{~mm}$, respectively.

tion 3.3 and Equation 3.14. Slight differences in the measurement curves of different tubes can be seen. The deviation from the model is large for the smaller wall thickness. To explain this let us consider the Biot number $\left(\mathscr{B} i=\frac{h \delta}{\lambda}\right)$. As the Biot number is proportional to the wall thickness, a reduction in the Biot number leads to simultaneous cooling of the inner and the outer wall of the tube and therefore increases the error introduced due to the Perfect Thermal Contact Assumption (PTCA) at the outer wall.

Having shown that PTCA and the validity of the approximate model in determining the transient temperature during the cool-down of a polypropylene tube in liquid nitrogen, the effect of a tissue attached to the inner wall of the tube is now studied. The tissue is assumed to be a lumped mass meaning no temperature gradients in the material. This is a fair assumption as more than $60 \%$ of the tissue is made up of water whose thermal conductivity is one or- 


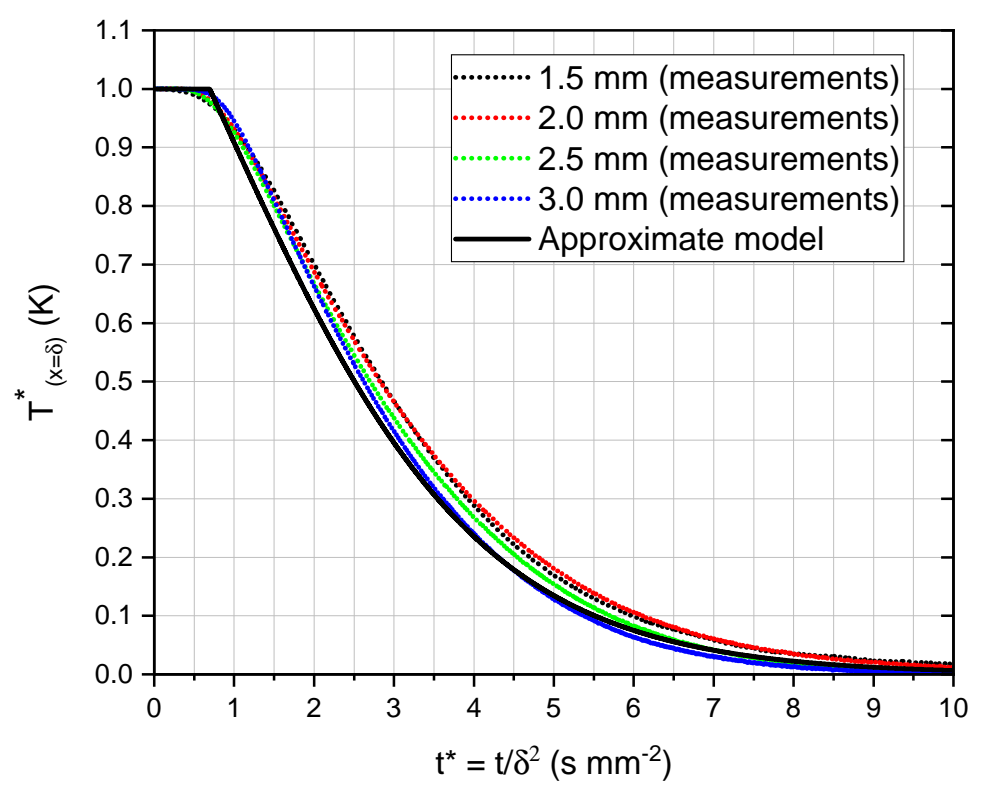

Figure (3.7) Comparison of the dimensionless temperature of the tube wall calculated using the approximate solution and measured data.

der higher than the polypropylene. The temperature variation of the tissue as a function of time for several heat capacity values of the tissue is calculated using Equation 3.12 and is shown in Figure 3.8a. The calculations are performed for wall thickness $\delta$ of $1.5 \mathrm{~mm}$ and initial temperature $T_{i}$ of $295 \mathrm{~K}$. In an interesting finding, the difference in the cool-down time of the tissue and inner wall of an empty tube, $\Delta t=t-t_{\left(C_{t}=0\right)}$ is proportional to the product of the thermal resistance in the vial wall $\left(R_{\text {wall }}=\frac{\delta}{\lambda A}\right)$ and the tissue heat capacity $\left(C_{t}\right)$. Figure $3.8 \mathrm{~b}$ shows the dimensionless time $\frac{\Delta t}{R_{\text {wall }} C_{t}}$ as a function of the cold temperature.

To understand this behavior, a simplified model is adopted as described here. The approximate solution is not used for the explanation as we cannot obtain an explicit expression of the cool-down time in terms of the tissue temperature. In this simplified model, the vial wall is assumed as a combination of a lumped mass and a thermal resistance. The equivalent thermal network diagram for the tissue and the vial is shown in Figure 3.9. 


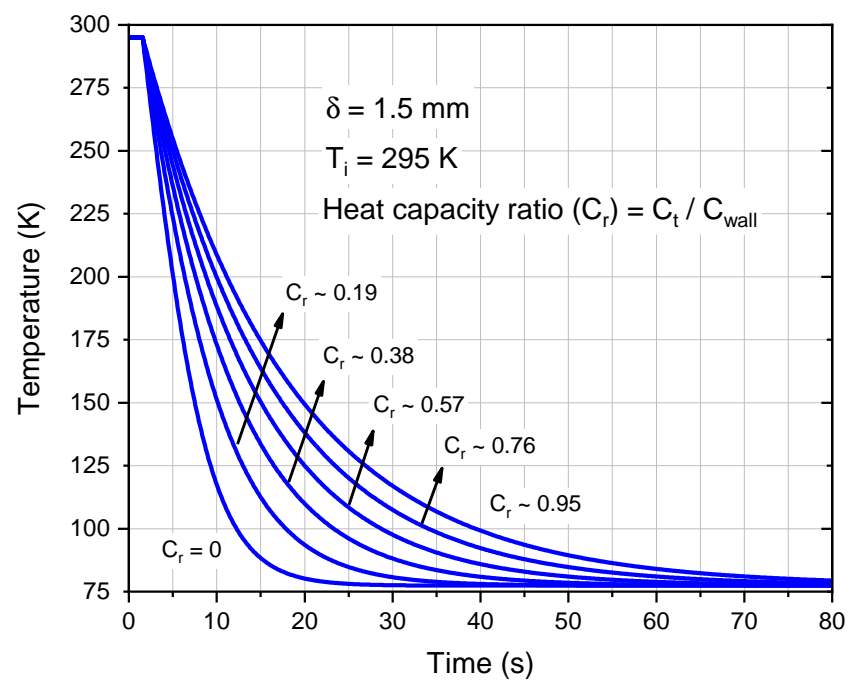

(a)

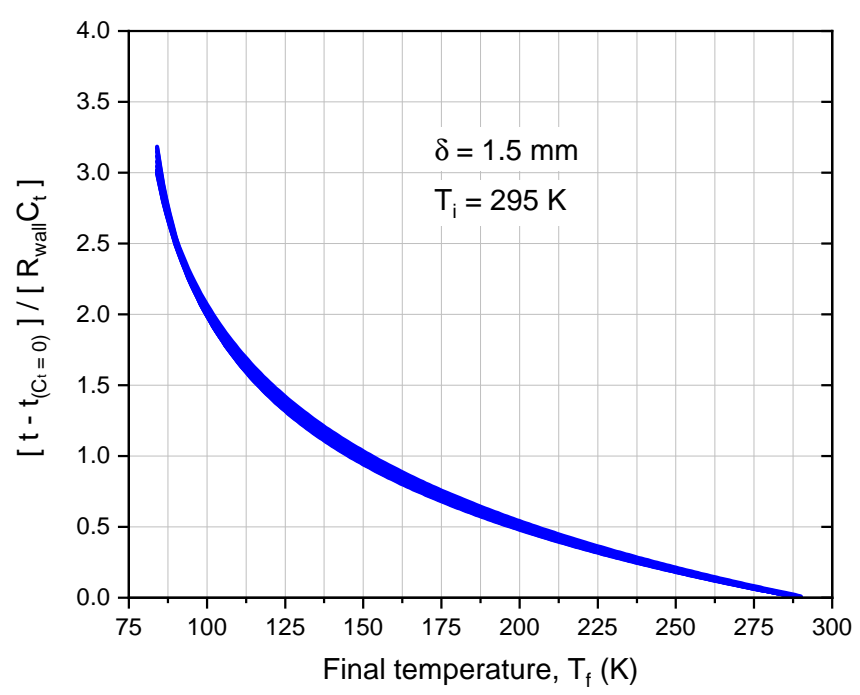

(b)

Figure (3.8) (a) Cooldown of a tissue for several heat capacity values (b) Ratio of the difference in the cooldown time of the tissue and the inner wall of an empty vial to the product of wall resistance and heat capacity of the tissue. 
The cool-down time for the tissue to reach any final temperature value $T_{f}$ is given by, $t=-\ln \left(\frac{T_{f}-T_{\infty}}{T_{i}-T_{\infty}}\right) R_{\text {wall }}\left(C_{\text {wall }}+C_{t}\right)$. The difference in the cool-down time values is $\Delta t=t-t_{\left(C_{t}=0\right)}=-\ln \left(\frac{T_{f}-T_{\infty}}{T_{i}-T_{\infty}}\right) R_{\text {wall }} C_{t}$. For the same initial $\left(T_{i}\right)$ and final $\left(T_{f}\right)$ temperature values we obtain $\frac{\Delta t}{R_{\mathrm{wall}} C_{t}}$ to be a constant value.

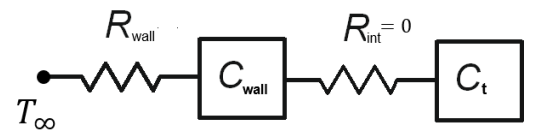

Figure (3.9) Simplified thermal network diagram of a tissue represented as a heat capacity attached to a tube wall.

This finding in particular will have a huge impact on drafting precise clinical protocols for tissue snap-freezing. A step-by-step procedure is elaborated here. Once a polypropylene tube is chosen, using Equation 3.14 the temperature of the inner wall of the tube as a function of time can be calculated. The only geometrical input parameter to this equation is the wall thickness of the tube. Knowing the tissue volume, its heat capacity can be calculated. Depending on the application, the end temperature might be of interest, using this value and the plot shown in Figure 3.8b the dimensionless time difference is obtained. From this time difference and the time calculated for an empty tube the time taken for a tissue to cool can be determined. This simple and elegant approach will lead to defining quantitative and reliable cooling protocols in tissue and other bio-material freezing.

\subsection{Conclusions}

We studied systematically thermal characteristics of the polypropylene tubes quenched in liquid nitrogen. We showed that the Perfect Thermal Contact Assumption (PTCA) is valid for the polypropylene material by comparing the numerical solution and experimental data. We also developed a onedimensional heat transfer model predicting the temperature variation inside the vial wall and of the tissue attached to it. This model also includes temperaturedependent thermal properties of the polypropylene and can be used for the vials of any shape and size provided a one-dimensional assumption for the vial-wall is valid. This requires the characteristic length representing the lat- 
eral dimension to be large compared to the vial-wall thickness. A fairly good prediction of the temperature variation at the inner wall of an empty tube is obtained with a maximum error of less than $10 \%$ compared to measurements. The cool-down time of the inner wall of an empty tube scales with the square of its wall thickness. The cooling of a tissue attached to the inner wall of the tube is also studied. A significant finding is that the difference in the cooldown time of a tissue in the tube and an empty tube is proportional to the product of the tissue heat capacity and the tube-wall thermal resistance. Therefore, using the results from our model, one can estimate the cool-down time of the tissue placed in a polypropylene tube immersed in liquid nitrogen. This approach will allow the development of improved and precise tissue freezing protocols for cryopreservation.

\section{Appendix - I}

\section{Approximate solution for cool-down of tissue enclosed in a polypropy- lene tube:}

For late regime, using Equation 3.2, Equation 3.9 is rewritten as,

$$
\underbrace{\left(\frac{-C_{t}}{\lambda A}\right)}_{A_{t}}\left[\left.\alpha(T(\delta, t)) \frac{\partial^{2} T}{\partial x^{2}}\right|_{x=\delta}\right]=\left.\frac{\partial T}{\partial x}\right|_{x=\delta}
$$

Now, using Equation 3.10 and Equation 3.11, temperature variation inside the polypropylene material is given by,

$$
T(x, t)=T_{\infty}+b x+d x^{3} \quad \text { in } \quad 0 \leq x \leq \delta, \quad t \geq t_{\delta}
$$

Using the value of $T(x, t)$ from Equation 3.16 in Equation 3.15, the relationship between the variables $b$ and $d$ is given by,

$$
b=\frac{d\left(C_{1}+C_{2} d\right)}{1-C_{3} d}
$$

in this, $C_{1}, C_{2}$ and $C_{3}$ are constants. The expression for $d$ is now calculated using Equation 3.2 where thermal diffusivity $(\alpha)$ of the polypropylene material is temperature-dependent and is approximated as, $\alpha(T)=\alpha_{1}+\beta_{1} T$ (for 
values of $\alpha_{1}$ and $\beta_{1}$ see Figure 3.3). Integrating Equation 3.2 with respect to $x$ from $x=0$ to $\delta$,

$$
\int_{x=0}^{\delta}\left(\alpha_{1}+\beta_{1} T\right) \frac{\partial^{2} T}{\partial x^{2}} d x=\int_{x=0}^{\delta} \frac{\partial T}{\partial t} d x
$$

and solving left side of the Equation 3.18,

$$
\begin{aligned}
\int_{x=0}^{\delta}\left(\alpha_{1}+\beta_{1} T\right) \frac{\partial^{2} T}{\partial x^{2}} d x & =\int_{x=0}^{\delta}\left(\alpha_{1}+\beta_{1}\left(T_{\infty}+b x+d x^{3}\right)\right)(6 x d) d x \\
& =3 d \delta^{2} \alpha\left(T_{\infty}\right)+2 \beta_{1} \delta^{3} b d+\frac{6}{5} \beta_{1} \delta^{5} d^{2} \\
& =\frac{C_{4} d+C_{5} d^{2}+C_{6} d^{3}}{1-C_{3} d} \quad \text { (using Equation 3.17) }
\end{aligned}
$$

in this, $C_{4}, C_{5}$ and $C_{6}$ are constants. Now, after multiplying Equation 3.19 by a factor of $\left(1-C_{3} d\right)^{2}$,

$$
\begin{aligned}
\left(1-C_{3} d\right)^{2}\left[\int_{x=0}^{\delta}\left(\alpha_{1}+\beta_{1} T\right) \frac{\partial^{2} T}{\partial x^{2}} d x\right] & =\left(1-C_{3} d\right)^{2}\left[\frac{C_{4} d+C_{5} d^{2}+C_{6} d^{3}}{1-C_{3} d}\right] \\
& =C_{4} d+C_{7} d^{2}+C_{8} d^{3}+C_{9} d^{4}
\end{aligned}
$$

in this, $C_{7}, C_{8}$ and $C_{9}$ are constants. Now, solving right hand side of the Equation 3.18,

$$
\int_{x=0}^{\delta} \frac{\partial T}{\partial t} d x=T_{\infty}+\frac{\partial b}{\partial t} \frac{\delta^{2}}{2}+\frac{\partial d}{\partial t} \frac{\delta^{4}}{4}
$$


Here, $\frac{\partial b}{\partial t}$ is calculated using Equation 3.17 and is given by,

$$
\frac{\partial b}{\partial t}=\left[\frac{C_{1}+2 C_{2} d-C_{2} C_{3} d^{2}}{\left(1-C_{3} d\right)^{2}}\right] \frac{\partial d}{\partial t}
$$

Now using Equation 3.22, Equation 3.21 is rewritten as,

$$
\left(1-C_{3} d\right)^{2}\left[\int_{x=0}^{\delta} \frac{\partial T}{\partial t} d x\right]=\left[I_{1}+I_{2} d+I_{3} d^{2}\right] \frac{\partial d}{\partial t}
$$

in this, $I_{1}, I_{2}$, and $I_{3}$ are constants. Now, by equating and integrating Equation 3.20 and Equation 3.23 with respect to time,

$$
\int_{t=t_{\delta}}^{t} \partial t=\int_{d=d_{i}}^{d} \frac{\left[I_{1}+I_{2} d+I_{3} d^{2}\right]}{\left[C_{4} d+C_{7} d^{2}+C_{8} d^{3}+C_{9} d^{4}\right]} \partial d
$$

the cool-down time for the tissue enclosed is given by,

$t-t_{\delta}=\frac{I_{1} \ln \left(\left|\frac{d}{d_{i}}\right|\right)}{C_{4}}-\frac{\sum_{\left\{: C_{9} Z^{3}+C_{8} Z^{2}+C_{7} Z+C_{4}=0\right\}} \frac{\left[\left(I_{1} C_{7}-I_{2} C_{4}\right)+\left(I_{1} C_{8}-I_{3} C_{4}\right) Z+\left(I_{1} C_{9}\right) Z^{2}\right] \ln \left(\left|\frac{d-Z}{d_{i}-Z}\right|\right)}{\left[C_{7}+\left(2 C_{8}\right) Z+\left(3 C_{9}\right) Z^{2}\right]}}{C_{4}}$

Approximate solution for empty polypropylene tube $\left(C_{t}=0\right)$ :

For $C_{t}=0$, the values of $C_{1}, C_{2}, C_{3}, C_{4}, C_{5}, C_{6}, C_{7}, C_{8}, C_{9}, I_{1}, I_{2}$ and $I_{3}$ are given by,

$$
\begin{array}{lll}
C_{1}=-3 \delta^{2} ; & C_{2}=0 ; & C_{3}=0 ; \\
C_{4}=3 \delta^{2} \alpha\left(T_{\infty}\right) ; & C_{5}=\frac{-24}{5} \beta_{1} \delta^{5} ; & C_{6}=0 ; \\
C_{7}=\frac{-24}{5} \beta_{1} \delta^{5} ; & C_{8}=0 ; & C_{9}=0 ; \\
I_{1}=\frac{-5 \delta^{4}}{4} ; & I_{2}=0 ; & I_{3}=0 ;
\end{array}
$$

Using these values, the value of $b$ is calculated using Equation 3.17 and is given by,

$$
b=-3 \delta^{2} d
$$


Equation 3.12 can now be re-written as,

$$
t-t_{\delta}=\frac{I_{1} \ln \left(\left|\frac{d}{d_{i}}\right|\right)}{C_{4}}-\frac{\sum_{\left\{Z: C_{7} Z+C_{4}=0\right\}} I_{1} \ln \left(\left|\frac{d-Z}{d_{i}-Z}\right|\right)}{C_{4}}
$$

Using this, the value of $d$ is given by,

$$
d=\frac{\frac{C_{4} d_{i}}{C_{4}+C_{7} d_{i}} e^{\left(\frac{C_{4}\left(t-t_{\delta}\right)}{I_{1}}\right)}}{1-\frac{C_{7} d_{i}}{C_{4}+C_{7} d_{i}} e^{\left(\frac{C_{4}\left(t-t_{\delta}\right)}{I_{1}}\right)}}
$$

in this, the value of $d_{i}$ is calculated using Equation 3.13 and is given by,

$$
d_{i}=\frac{T_{\infty}-T_{i}}{2 \delta^{3}}
$$

Now, using the values of $b$ and $d$ from Equation 3.24 and Equation 3.26 respectively, the temperature variation during the late regime is calculated using equation Equation 3.16 and is given by,

$$
T^{*}\left(x, t^{*}\right)=\frac{T\left(x, t^{*}\right)-T_{\infty}}{T_{i}-T_{\infty}}=\frac{\frac{3}{2} \alpha\left(T_{\infty}\right)\left[\frac{x}{\delta}-\frac{1}{3}\left(\frac{x}{\delta}\right)^{3}\right] e^{\frac{-12}{5} \alpha\left(T_{\infty}\right)\left(t^{*}-\frac{1}{8 \alpha(\bar{T})}\right)}}{\alpha\left(T_{\infty}\right)+\frac{4}{5} B\left(T_{i}-T_{\infty}\right)\left[1-e^{\frac{-12}{5} \alpha\left(T_{\infty}\right)\left(t^{*}-\frac{1}{8 \alpha(\bar{T})}\right)}\right]}
$$




\title{
How to estimate minimum cool-down time for insulated metals in liquid nitrogen? ${ }^{1}$
}

\begin{abstract}
The cooling rate of metallic objects quenched in liquid nitrogen can be enhanced by coating its surface with a material that has a low thermal effusivity. An early transition from film to nucleate boiling regime caused due to the formation of cold spots at the liquid-coating interface is reported as the reason for this enhanced cooling rate. However, until now, optimization of the coating thickness to minimize the overall cooling time has only been an empirical proposition. Inspired by experimental data a phenomenological model is proposed. Using this model, an approximate insulation coating thickness that will approach the fastest cool down of an insulated metal quenched in liquid nitrogen can be predicted. This model is verified with experimental data of several copper cylinders coated with different thickness of epoxy quenched in saturated as well as sub-cooled liquid nitrogen. The optimum coating thickness reduces significantly with the degree of liquid sub-cooling.
\end{abstract}

\subsection{Introduction}

Thermal quenching in a liquid pool is a common and convenient laboratory and industrial process, spanning a wide range of applications. To name a few, in cryopreservation of biological samples where plastic tubes containing the sample are quenched in liquid nitrogen [13], in material processing such as quenching tool steel in a liquid nitrogen bath to improve hardness [37], in various cryogenic systems where the connecting pipelines must be first chilled

\footnotetext{
${ }^{1}$ Published as : Jagga, S. and Vanapalli, S., 2020. Cooldown of insulated metals in saturated and subcooled liquid nitrogen. Cryogenics, 109, p.103114.
} 
$[38,39]$.

The boiling heat transfer in saturated liquid nitrogen is classified into three regimes, namely the film, transition, and nucleate boiling [46] depending on the wall superheat temperature $\left(\theta=T-T_{f}\right)$. At high wall superheat the vapour generated forms a layer between the wall and liquid, resulting in a limited heat transfer to the liquid. The thickness of the vapour layer reduces with the reduction in wall superheat $\left(\theta=T-T_{f}\right)$ and breaks, when the wall temperature drops below the minimum film boiling temperature. After this, a transition to the nucleate boiling regime occurs, where due to increased liquidsolid contact, higher heat flux is obtained. However, these regimes are elucidated only to the materials with high thermal effusivity, while materials with a low thermal effusivity shows early transition from film to the nucleate boiling regime [40]. The thermal effusivity $(\varepsilon=\sqrt{\lambda \rho c})$ of a material is a measure of the materials ability to exchange thermal energy with its surroundings. The early transition in the boiling regime is suspected due to an early drop in the outer wall temperature.

In a classical observation, Cowley et. al. [41] showed that the application of a thin layer insulated material to a body will decrease the cooldown time of a metallic object. Later a number of studies are performed to pin the underlying mechanisms that led to this counter intuitive result, which are briefly reviewed here. Cowley et. al. [41], showed significant improvement in the cool-down time (from $25^{\circ} \mathrm{C}$ to $-196^{\circ} \mathrm{C}$ ) of an aluminum cylinder coated with different thickness of vaseline material. They also observed that a minimum value of the cool-down time exist for an optimum thickness of the coating. Similar experiments are also performed by Maddox et. al. [42], in which teflon is used as a coating material on stainless steel 304 tubes. However, the analysis reported was inconclusive due to the unknown values of the surface temperature of the coating material. The authors recommended to rely on the experimental data for evaluating the optimum coating thickness corresponding to the minimum cool-down time. Manson [43] suggested that the theories based on average values of heat flux and wall super-heat will not explain the early transition in the boiling regime. The author proposed that a periodic and spatially nonuniform heat-transfer mechanism occurs at the liquid-coating interface, which due to low thermal effusivity of the coating material would result in the formation of cold spots on the coating surface, thus causes early collapse of stable 
film boiling. Experiments of coated surfaces in sub-cooled liquid nitrogen is reported recently by Chung et. al. [58, 59]. However, no quantitative analysis was performed to predict the early transition for coated metals. Moreaux et. al. [40] reported that the low thermal effusivity of the coating material is responsible for the existence of Larvate boiling during quenching. Larvate boiling is an intermediate mode between film and nucleate boiling [40]. Kikuchi et. al. [44] also suggested that a non-uniform heat-transfer mechanism takes place at the liquid-coating interface. The authors assumed that the heat transfer at the liquid-coating interface occurs due to a series of repeated heat transfer phenomenon caused by thermal diffusion, film and nucleate boiling in the liquid domain. A one-dimensional analytical model capturing the heat transfer aspects of the coated metals quenched in liquid nitrogen is also reported. However, the model uses an empirical approach to determine the time scales for different heat transfer phenomenons (thermal diffusion, film and nucleate boiling).

In conclusion, previous studies suggested local cooling of the surface to the early collapse of the film, although no microscale heat transfer studies are reported to support this claim. From an application perspective, it is clear that an insulation coating decreases the cool down time, however, a tool to predict an optimum coating thickness based on the thermal properties of the material is still lacking. In this paper, we address this gap by presenting an approximate predictive model of the coating thickness based on the thermal properties and the pool temperature. We present experimental data of the cooling of several epoxy coated copper cylinders in saturated and sub-cooled liquid nitrogen. The heat flux and cooldown time derived from these experiments showed interesting trends, which allowed us to develop an approximate model to predict the optimum coating thickness. The heat transfer model is also validated using the measurements and its general applicability is also discussed.

\subsection{Materials and methods}

Seven similar copper cylinders of length $(L)$ and diameter $(D)$ of $60 \mathrm{~mm}$ and 6 $\mathrm{mm}$, respectively are used for the experiments. The high aspect ratio $\left(\frac{L}{D}=10\right)$ is chosen to neglect the heat transfer from the side surfaces of the cylinder as the ratio of the lateral and longitudinal area, $A_{\text {ratio }}=\frac{2\left(\pi D^{2} / 4\right)}{(\pi D L)}=0.05$, is low. The longitudinal area of the cylinders are coated with an epoxy (stycast 
1266) material to different coating thickness $(\delta)$ values of $50 \mu \mathrm{m}, 100 \mu \mathrm{m}$, $150 \mu \mathrm{m}, 200 \mu \mathrm{m}, 300 \mu \mathrm{m}$, and $500 \mu \mathrm{m}$. The coating procedure is as follows, first the copper cylinder is machined to a diameter of $6 \mathrm{~mm}$. Next the copper cylinders are placed in a glass tube with about $1 \mathrm{~cm}$ of the cylinder sticking out of the tube. Epoxy is filled in the gap formed between the cylinder and the glass tube, which is about $2 \mathrm{~mm}$. After hardening of the epoxy, the glass tube is sacrificed. The cylinder is mounted in a turning tool and the epoxy is machined to the required coating values. All the cylinders are prepared with the same batch of epoxy. The temperature of the cylinder is measured at its center using a type-E thermocouple, which is soldered at the inner end of the hole made at the centre of the cylinder (see Figure 4.1). The diameter and length of the hole is $1.2 \mathrm{~mm}$ and $3 \mathrm{~mm}$. Liquid nitrogen pool used for the experiments is pre-cooled to different temperature values of $66 \mathrm{~K}, 70 \mathrm{~K}, 74 \mathrm{~K}$ by evaporative cooling, and is kept open to the atmosphere during experimentation. The heat transfer from the cylinder during its cool-down is about $1 \mathrm{~kJ}$. The specific heat capacity of the bath is about $10 \mathrm{kJK}^{-1}$. Therefore, the bath temperature will not increase substantially during the quenching process. The cylinder is immersed horizontally at room temperature and is maintained to approximately the same depth in the liquid nitrogen pool.

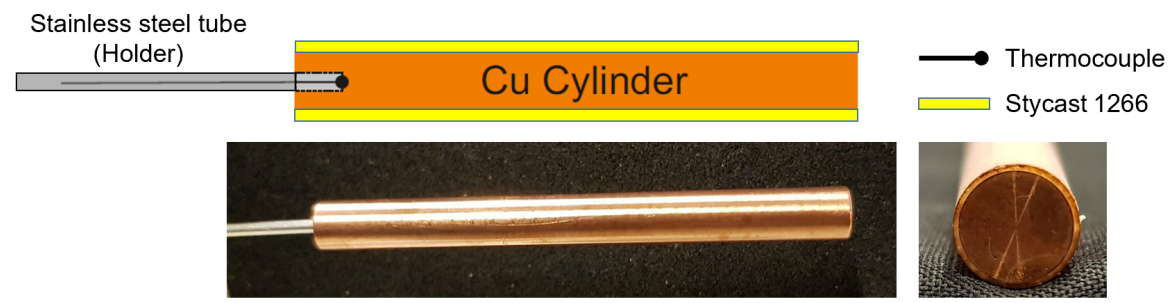

Figure (4.1) Schematic and photos of a coated cylinder. A stainless steel tube with outer diameter and wall thickness of $1.2 \mathrm{~mm}$ and $0.1 \mathrm{~mm}$, respectively is used to hold the cylinder in the liquid cryogenic pool.

The cylinder is held stationary during the measurements using a thin stainless steel tube of $1.2 \mathrm{~mm}$ outer diameter and $0.1 \mathrm{~mm}$ wall thickness glued to the side face of the cylinder. The length of the stainless steel tube inside the liquid pool is at approximately $10 \mathrm{~cm}$ for each experiment. Due to the low heat capacity of the tube, it cools faster compared to the cylinder and therefore the parasitic heat load through the tube is neglected in the present 
analysis. The large length of the tube inside the liquid pool also helps in further reducing the parasitic heat load through the tube. The measurements are recorded at the rate of 100 samples per second. To demonstrate the repeatability, three independent experimental runs were conducted for each measurement. Thermal properties of the stycast are assumed to be constant, its density $(\rho)$, specific heat capacity $(c)$ and thermal conductivity $(\lambda)$ are $1120 \mathrm{kgm}^{-3}$ [60], $1000 \mathrm{Jkg}^{-1} \mathrm{~K}^{-1}$ [61] and $0.18 \mathrm{Wm}^{-1} \mathrm{~K}^{-1}$ [61], respectively. The specific heat capacity and thermal conductivity of the copper material is temperature dependent and are given by $c=\left(-1.35 \times 10^{-7} T^{4}+1.29 \times 10^{-4} T^{3}-\right.$ $\left.4.73 \times 10^{-2} T^{2}+8.23 T-2.15 \times 10^{2}\right) \mathrm{Jkg}^{-1} \mathrm{~K}^{-1}$ for $60 \mathrm{~K}<T<300 \mathrm{~K}$ [30] and $\lambda=\left(-1.26 \times 10^{-5} T^{3}+8.97 \times 10^{-3} T^{2}-2.11 T+5.55 \times 10^{2}\right) \mathrm{Wm}^{-1} \mathrm{~K}^{-1}$ [31] for $100 \mathrm{~K}<T<300 \mathrm{~K}$ respectively. The density of the copper is 8952 $\mathrm{kgm}^{-3}[62]$.

\subsection{Results and discussion}

The temperature data during the cooling of the bare and the coated cylinders in saturated and sub-cooled liquid nitrogen pool is shown in Figure 2. We observe the overall cool down time of the bare cylinder reduces as the degree of sub-cooling increases, similar to quenching results in water [63]. The coated cylinders also exhibit similar behavior. In addition, the cooling time in the case of a coated cylinder shows a strong dependency on the coating thickness for a particular pool temperature. As the coating thickness is increased, the cooling time reduces at first and beyond a certain value increases. Similar observations were also made by Cowley et. al.[41] and Maddox et. al. [42] in a pool of saturated liquid nitrogen. The coating thickness leading to the lowest cool down time also depends on the pool temperature as noted in our experimental data, which is not studied previously.

To draw insights into the physical processes in these experiments, the heat flux (q) is calculated from the temperature-time data. The copper cylinder can be assumed as a lumped mass because the Biot number representing the ratio of the internal to the external thermal resistance is approximately 0.07 corresponds to the lowest thermal conductivity value of copper at room temperature $\left(385 \mathrm{Wm}^{-1} \mathrm{~K}^{-1}\right)$ and maximum heat transfer coefficient value of $10^{4} \mathrm{Wm}^{-2} \mathrm{~K}^{-1}$ and the Fourier number is greater than one after 0.10 seconds. The $q$ and $h$ values are calculated using, 


$$
q=h\left(T-T_{f}\right)=-\frac{m c}{A} \frac{d T}{d t}
$$

in this, $m$ and $c$ are the mass and specific heat capacity of the copper cylinder. The cooling rate $\left(\frac{d T}{d t}\right)$ is calculated from the measurements using a second order Savitzky-Golay method [64] with 21 neighboring points. The heat transfer area $(A)$ for the copper cylinder is approximated as, $A=\pi D L$. This approximation is valid due to the low coating thickness $(\delta)$ values compared to the diameter $(D)$ of the cylinder. The heat transfer through the side faces is also neglected. The calculated values of the heat flux for all cases are shown in Figure 4.3.

The heat flux as a function of surface temperature curve for the bare cylinder is similar to the classical pool boiling curve [49], which consist of three different boiling regimes; starting from the film regime at warm surface temperature, followed by transition and nucleate regimes. The magnitude of the heat flux increases as the pool temperature is reduced, which is similar to the findings reported in literature [65] and is attributed to the re-condensation of vapors in the sub-cooled liquid pool. Although similar regimes are visible for coated cylinders, the temperature span of the film regime reduces as the coating thickness is increased followed by complete disappearance of the regime beyond a certain thickness value.

In an interesting finding, the calculated critical heat flux (CHF) or the maximum heat flux in nucleate regime values is fairly independent of the coating thickness value and this trend is observed for all the pool temperatures. Similar trend is also observed in the experimental results reported by Hellmann et. al. [49] for several coated cylinders in saturated liquid nitrogen. The physical reason for this observation is unknown and further studies are required to pin the underlying mechanisms. The calculated $\mathrm{CHF}\left(q_{\mathrm{CHF}}\right)$ values for the bare cylinder in liquid pools with saturated and pre-cooled to $74 \mathrm{~K}, 70 \mathrm{~K}$ and $66 \mathrm{~K}$ are approximately $1.2 \mathrm{Wm}^{-2}, 1.4 \mathrm{Wm}^{-2}, 1.75 \mathrm{Wm}^{-2}$ and $2.2 \mathrm{Wm}^{-2}$, respectively. These values will be used later in the approximate model. 

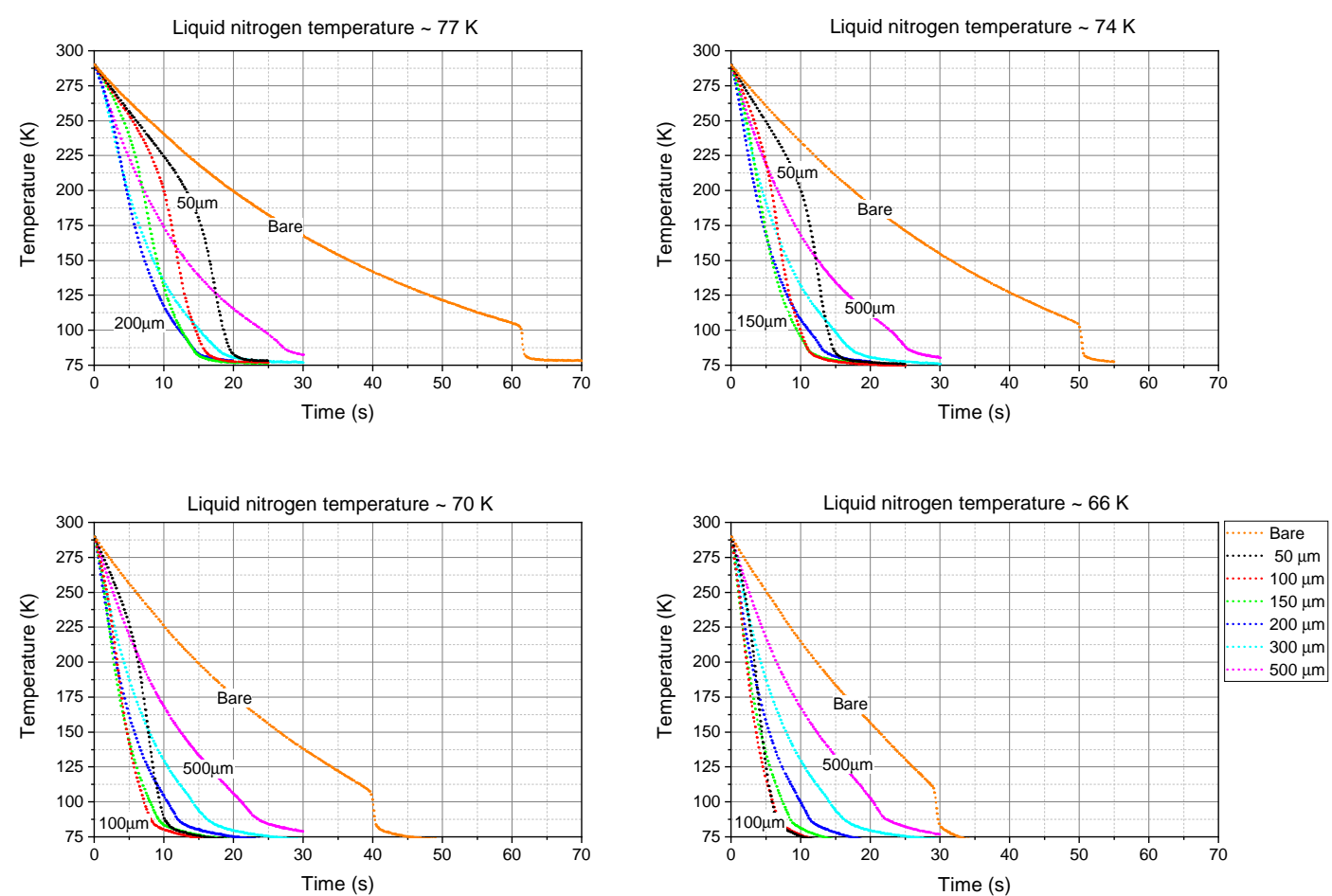

Figure (4.2) Temperature measurements of the bare and coated copper cylinders in saturated and subcooled liquid nitrogen. Temperature is measured using a type-E thermocouple with an accuracy of $\pm 2 \mathrm{~K}$. The legend shown is applicable to all the sub-plots. 

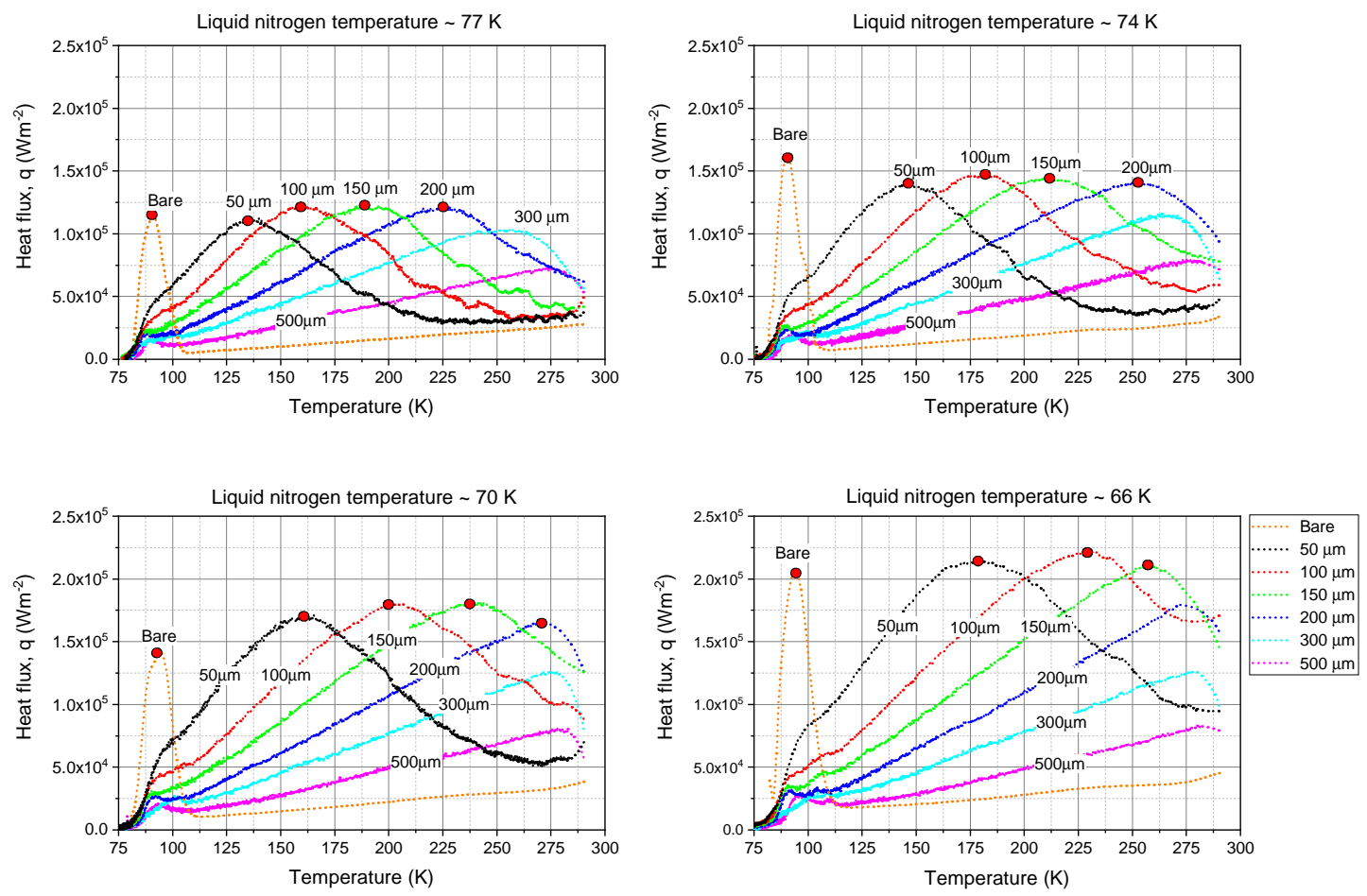

Figure (4.3) Calculated heat flux from the temperature-time measurements using equation 4.1 as a function of metal temperature for all the cylinders quenched in saturated and subcooled liquid nitrogen. The points denote the critical heat flux values and the corresponding metal temperature. The legend shown is applicable to all the sub-plots. 


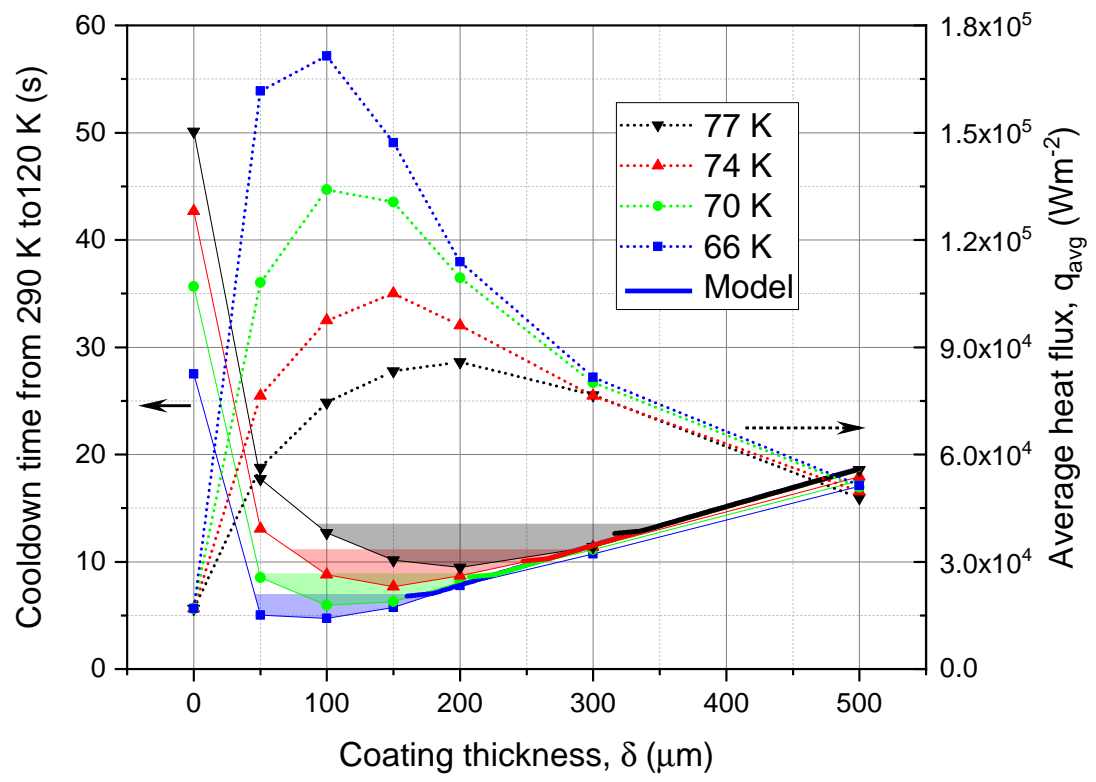

Figure (4.4) Cool-down time from $290 \mathrm{~K}$ to $120 \mathrm{~K}$ and the corresponding average heat values. The points are joined by straight lines for better visualization. The predicted values of cool-down time in the nucleate boiling regime are also shown. The shaded area represents the values for which the cool-down time is less than $10 \%$ of the minimum value corresponding to the optimum coating thickness.

As we noticed in Figure 4.2, the cool down time first reduces to a lowest value and increases again as the coating thickness is increased. In Figure 4.4, we plot the cool-down time of the cylinder from $290 \mathrm{~K}$ to $120 \mathrm{~K}$ for all the cases. This temperature range is chosen due to our sample constraints. The heat flux due to boiling on the side surfaces of the cylinder that are not covered with the epoxy coating will increase substantially below $120 \mathrm{~K}$. In the same plot, temperature averaged heat flux is also shown. The average is the integral of the heat flux with temperature divided by the temperature range. For a particular pool temperature the average heat flux reaches a maxima for a coating thickness. As expected, the minimum cool down time corresponds to this maximum average heat flux. Tracing these curves from left to right, for small coating thickness values the average heat flux is low due to the presence of 
the film boiling regime in the cooling temperature span. The average heat flux peaks for a certain coating thickness as the film boiling regime contribution decrease. Further decrease in the average heat flux is due to the finite thermal resistance of the coating, which increases as the coating thickness increases. Kikuchi et al. [44] approached this problem by developing an empirical microscale heat transfer model using fitting parameters to explain the optimum coating thickness. Instead, we adopt a different method, by approaching the optimum case from the right side i.e. from higher coating values to the optimum case. We will not be able to model the optimum case which is the defined as the coating value for the lowest cool down time, but close to it. The details of the model is discussed in the next section.

\subsubsection{Phenomenological modeling}

In this section an approximate physical model based on experimental observations to determine the optimum coating thickness is discussed. This model will incorporate pool temperature and thermal properties of the coating. In Figure 4.5, the calculated heat flux and heat transfer coefficient values for a bare and a $100 \mu \mathrm{m}$ coated cylinder is shown. This particular coated cylinder is chosen as a representative case. It is interesting to note that for small super heat temperatures the characteristics of the heat transfer coefficient of the bare and the coated cylinders are different.

The heat flux (q) curve is divided into three different sections, namely AB, $\mathrm{BC}$ and $\mathrm{CD}$, representing nucleate, transition, and film boiling regimes, respectively as shown in Figure 4.5. The nucleate boiling regime is defined for the temperature values lower than the temperature corresponding to the critical heat flux $\left(\mathrm{T}_{\mathrm{CHF}}\right)$. For convenience, discussion of this nucleate regime is limited to temperature values greater than $100 \mathrm{~K}\left(\theta_{m} \approx 25 \mathrm{~K}\right)$. As discussed above, bare side faces of the copper cylinder goes through the transition to the nucleate boiling regime, due to which heat flux through the edges increases and therefore, its contribution in the total heat flux (q) values becomes visible (see Figure 4.3). To simplify the discussion, nucleate boiling regime (section $\mathrm{AB}$ ), is further divided into two different sections $\mathrm{AA}_{1}$ and $\mathrm{A}_{1} \mathrm{~B}$, which will be discussed individually in the subsequent sections. 

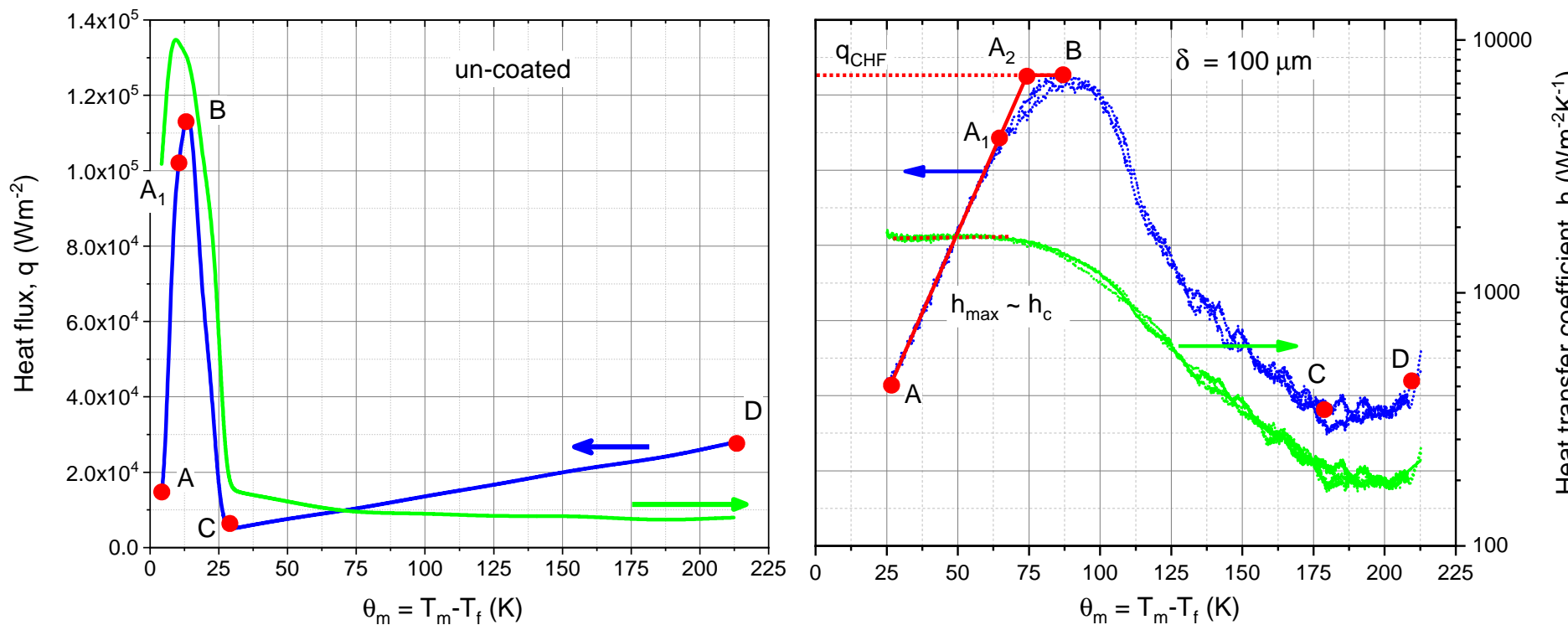

Figure (4.5) Calculated heat flux values for the bare (left) and coated cylinder with $100 \mu \mathrm{m}$ coating thickness (right) quenched in saturated liquid nitrogen pool. Model predicted heat flux in the nucleate regime for the coated case is also shown. 


\section{Section $A A_{1}$}

The heat transfer coefficient $(h)$ for the coated cylinder case in the section $\mathrm{AA}_{1}$ is fairly constant. Similar trend is also observed for all coated cylinder measurements. On the other hand, the heat flux increases with superheat temperature in the bare cylinder. The point $A_{1}$ is the inflection point, which is reported in the literature as the heat flux value corresponding to the maximum boiling heat transfer coefficient. The heat transfer process is characterized by the formation of isolated bubbles in section $\mathrm{AA}_{1}$ and by bubble jets and columns in section $\mathrm{A}_{1} \mathrm{~B}$ [66].

This difference in the heat transfer characteristics of the coated cylinder compared to the bare case can be best understood by considering an equivalent thermal network, which is shown in the Figure 4.6. The following assumptions are made:

- The copper cylinder is a lumped thermal capacitance.

- The heat capacity of the coating is negligible compared to the copper cylinder; this assumption is valid as for the cylinder with $500 \mu \mathrm{m}$ coating thickness value, the heat capacity of stycast is roughly $6 \%$ compared to the copper at room temperature.

We will later prove that these assumptions are valid for the problem under consideration. The effective thermal resistance $(R)$ between the liquid and the metal is the summation of the boiling resistance $\left(R_{b}\right)$ and that of the coating $\left(R_{c}\right)$,

$$
R=R_{b}+R_{c}=\frac{T_{m}-T_{f}}{q A}
$$

By re-writing the thermal resistance $\left(R=\frac{1}{h A}\right)$ in terms of heat transfer coefficient $(h)$,

$$
h=\frac{h_{c}}{1+\frac{h_{c}}{h_{b}}}=\frac{q}{T_{m}-T_{f}}
$$

In this, $T_{m}$ and $T_{f}$ are the temperature of the copper and liquid nitrogen pool, respectively and $h_{b}$ is the boiling heat transfer coefficient. The heat transfer coefficient due to the coating $\left(h_{c}\right)$ is calculated for the cylindrical shell 
with inner and outer diameter of $D$ and $D+2 \delta$, respectively and is given by, $h_{c}=\frac{2 \lambda_{c}}{D} \frac{1}{\ln \left(1+\frac{2 \delta}{D}\right)}$, where, $\delta$ and $\lambda_{c}$ is the thickness and thermal conductivity of the coating, respectively.

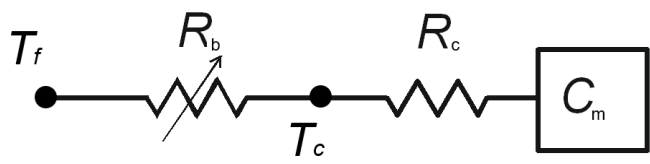

Figure (4.6) Equivalent thermal network diagram of the coated cylinder.

This two series resistance model suggest that in order to obtain an effective constant heat transfer coefficient the boiling heat transfer coefficient $h_{b}$ should be significantly high compared to the coating heat transfer coefficient $h_{c}$. The calculated value of boiling heat transfer coefficient at inversion point $\left(\mathrm{A}_{1}\right)$ for the bare cylinder in saturated liquid nitrogen pool is approximately $10^{4} \mathrm{Wm}^{-2} \mathrm{~K}^{-1}$, which is at-least 5 times high compared to the heat transfer coefficient offered by the coating material, $h_{\text {coating }} \approx 1829 \mathrm{Wm}^{-2} \mathrm{~K}^{-1}, 1229$ $\mathrm{Wm}^{-2} \mathrm{~K}^{-1}, 929 \mathrm{Wm}^{-2} \mathrm{~K}^{-1}, 629 \mathrm{Wm}^{-2} \mathrm{~K}^{-1}$ and $389 \mathrm{Wm}^{-2} \mathrm{~K}^{-1}$ for coating thickness $100 \mu \mathrm{m}, 150 \mu \mathrm{m}, 200 \mu \mathrm{m}, 300 \mu \mathrm{m}$ and $500 \mu \mathrm{m}$, respectively. Due to the low thermal effusivity of the coating material, the high value of boiling heat transfer coefficient at point $A_{1}$ further reduces the temperature of the liquid-coating interface to a value close to the liquid temperature. This results in enhanced liquid-solid contacts and therefore further reduces the thermal resistance offered by the liquid during the cooling period from point $\mathrm{A}$ to $\mathrm{A}_{1}$. In conclusion, heat transfer rate in the section $\mathrm{AA}_{1}$ is majorly limited by the coating material and therefore, a constant heat transfer coefficient value, which is equal to the heat transfer coefficient provided by the coating material i.e. $h \approx h_{c}$ can be used to predict the heat flux.

\section{Section $A_{1} B$}

The maximum heat flux in section $\mathrm{A}_{1} \mathrm{~B}$ is the Critical Heat Flux (CHF). The CHF is limited by the restricted liquid-solid contacts caused due to the formation of bubble columns resulting from the departing bubbles [66]. The CHF value for an object quenched in a liquid pool is a function of many parameters that includes, object dimensions, thermal properties, orientation, liquid properties [67, 68]. Liang and Mudawar [67] in their work has accessed dif- 
ferent models and correlations to predict CHF. The reported models and the correlations are able to predict the CHF values with a minimum error of approximately $20 \%$. In the present work, the CHF value is a deterministic parameter to estimate the minimum cool-down time, this will be discussed in detail later. Therefore, to avoid the error in the cool-down time estimation, CHF value from experimental data of the bare cylinder is used in the present analysis. The CHF value for the coated cylinder is also same for different coating thickness as discussed before.

The heat transfer coefficient values in the section $\mathrm{A}_{1} \mathrm{~B}$ is lower than the section $\mathrm{AA}_{1}$ and is unknown for the present configuration. If the same value of heat transfer coefficient is assumed for the entire nucleate boiling regime (section $\mathrm{AB}$ ), the temperature corresponds to the $\mathrm{CHF}$ will be given by point A2 instead of B. This will result in slightly different optimum coating thickness value, as it is assumed that the optimum coating thickness occurs when temperature corresponds to the $\mathrm{CHF}$ is equal to the room temperature; this is assumed due to the fact that a minimum cool-down time can be achieved by minimizing the thermal resistance offered to the copper material. The minimum possible thermal resistance value is the thermal resistance offered by the coating material only; this is only possible when thermal resistance due to the boiling fluid is either zero or is very low compared to the resistance of the coating material. Since thermal resistance due to the boiling is minimum in the nucleate boiling regime, it is, therefore, important to stay in the nucleate boiling regime during the entire cool-down to minimize the cool-down time.

Therefore, to predict heat flux in the section $\mathrm{A}_{1} \mathrm{~B}$, it is divided into two subsections $-A_{1} A_{2}$ and $A_{2} B$. The heat flux in the section $A_{1} A_{2}$ are estimated using the same constant heat transfer coefficient values $\left(h \approx h_{c}\right)$ as obtained in section $A_{1} A_{2}$ and the heat flux in the section $A_{2} B$ is assumed constant as shown in Figure 4.5. This approximation will result in slightly higher heat flux values for the section $\mathrm{A}_{1} \mathrm{~B}$, however this will not introduce large difference in the overall heat flux profile for the nucleate boiling regime, as the heat flux in this regime is limited by the critical heat flux value.

The point $\mathrm{B}$ corresponding to the $\mathrm{CHF}$ temperature moves to a larger superheat temperature as the coating thickness is increased. From this trend, we developed a strategy to determine a coating thickness when the CHF is at the initial immersion temperature of the cylinder $T_{\mathrm{m}, \mathrm{CHF}}$. From the measurement 
data for bare copper cylinders, the metal temperature $T_{\mathrm{CHF}}$ values corresponding to the CHF obtained are approximately $90 \mathrm{~K}, 92 \mathrm{~K}, 95 \mathrm{~K}$ and $100 \mathrm{~K}$ for liquid pool temperature of $77 \mathrm{~K}, 74 \mathrm{~K}, 70 \mathrm{~K}$ and $66 \mathrm{~K}$, respectively. In order to extend this to the coated cylinders we assume the coated surface in contact with the liquid will be at the above mentioned temperature when the CHF state is reached. This assumption can be verified from the results reported by Moreaux et. al. [40], where, a $2 \mu \mathrm{m}$ coating thickness of polymeric resin does not have much influence over the minimum film boiling temperature. For such a low coating thickness value, the temperature gradient inside the coating material are negligible and therefore temperature at the liquid-coating interface can be assumed same as the metal temperature. For negligible influence over the minimum film boiling temperature, the temperature corresponds to the $\mathrm{CHF}$ will also be close to the value for the bare metal case, as the temperature span after the minimum film boiling temperature is very low $(\approx 30 \mathrm{~K})$ and close to the bare metals in liquid nitrogen. Therefore, the coating thickness when the $\mathrm{CHF}$ is at the initial immersion temperature is estimated from the following expression,

$$
T_{\mathrm{m}, \mathrm{CHF}}=T_{\mathrm{CHF}}+R_{c} Q_{\mathrm{CHF}} .
$$

The estimated coating thickness value for which, $T_{\mathrm{m}, \mathrm{CHF}}=290 \mathrm{~K}$, are 315 $\mu \mathrm{m}, 265 \mu \mathrm{m}, 207 \mu \mathrm{m}$ and $159 \mu \mathrm{m}$ for liquid pool temperature of $77 \mathrm{~K}$, $74 \mathrm{~K}, 70 \mathrm{~K}$ and $66 \mathrm{~K}$, respectively. This means, the cylinder with coating thickness larger than these values will only be exposed to the nucleate boiling regime during its entire cool-down. The coating thickness value estimated by the approach described above for saturated liquid nitrogen is $315 \mu \mathrm{m}$, which is larger than the coating thickness value for a minimum cooldown time (see figure Figure 4.4). It is important to note that the optima is rather broad. By using our approach where only the nucleate boiling regime is considered the cool-down time reduction with a coating compared to the bare case is approximately $90 \%$ of the maximum possible time reduction. The exact prediction of the optimum coating thickness would require the heal flux prediction for the transition boiling regime. The experimental data reported for the transition boiling regime is very limited and so far there is no comprehensive theory or correlation to predict heat flux in this regime. 


\subsubsection{Validation of assumptions in the model}

Here we will consider a more generalized heat transfer model in which the coating heat capacity is included. We will compare the results from this model for two cases; first, for the case where heat capacity of the coating is included, which is referred hereafter as 'transient' and the second case where the heat capacity of the coating is set to a value two orders in magnitude lower than the actual case, which is referred hereafter as 'steady'. Moreover, we will make use of a similar heat transfer problem described in the book of Ozisik et. al. [57]. The geometry and the heat transfer boundary conditions representing our problem is shown in Figure 4.9 and for completeness the problem description, boundary conditions and the solution is given in the appendix. The comparison is performed for a coating thickness value of $500 \mu \mathrm{m}$, which is the maximum coating thickness value used for the experiments. Our aim is to discuss the error introduced in calculating the heat flux at the metal-coating interface, caused by neglecting the heat capacity of the coating material.

In order to replicate our earlier observations in this paper where the outer coating temperature may be assumed to be equal to the liquid temperature, we use a high value of heat transfer coefficient from the coating to the liquid pool. The heat transfer at the metal and coating interface is given by,

$$
Q_{\text {transient }}=-\left.\lambda_{c} \pi D L \frac{\partial \theta_{c}}{\partial r}\right|_{r=r_{m}}
$$

Figure 4.7 shows the heat transfer $Q_{\text {transient }}$ for a $500 \mu \mathrm{m}$ coating and in the same figure $Q_{\text {steady }}$ is shown. This data is obtained by setting a specific heat capacity value two orders in magnitude lower than the actual coating material value. The difference in the heat transfer rate for the two cases is plotted in Figure 4.7. The difference is maximum at room temperature, which is due to the limited thermal diffusivity inside the coating material. In the early regime, defined as the time taken for the cooling front to reach the metal and the coating interface, there is no heat transfer between the metal and coating. This is only pertinent for the transient case. In the steady case, $Q_{\text {steady }}$ is directly proportional to the temperature difference between the two interfaces, the metal-coating and coating-liquid. The constant slope of heat and temperature confirms this characteristic. 
Looking closely at the shape of the $Q_{\text {transient }}$, from room temperature the heat transfer increases and then follows the steady characteristics. The slope of the $Q_{\text {transient }}$ with temperature is lower than the $Q_{\text {steady }}$. In order to understand this characteristic let us investigate the temperature profile in the coating, which is given by [45],

$$
\theta_{c}(r, t)=\theta_{m}\left(r_{m}, t\right)\left[1-\frac{\ln \left(\frac{r}{r_{m}}\right)}{\ln \left(1+\frac{\delta}{r_{m}}\right)}\right] \quad \text { in } \quad r_{m} \leq r<\left(r_{m}+\delta\right), \quad t>0
$$

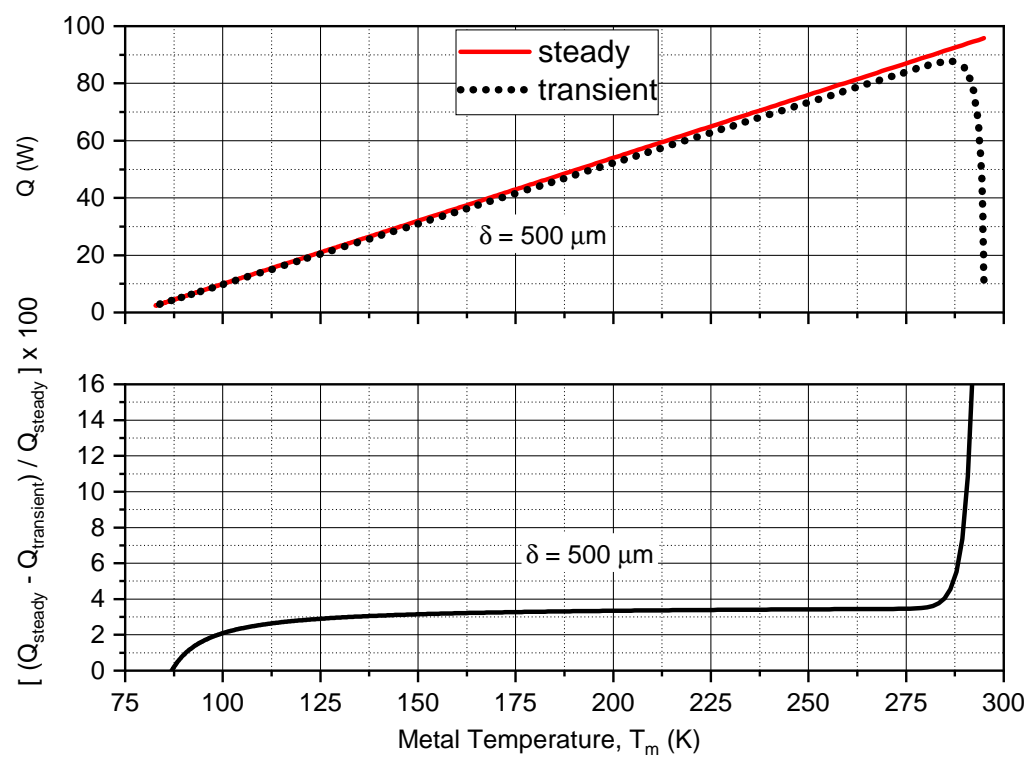

Figure (4.7) Heat transfer to the metal with and without considering the heat capacity of the coating (top) and the corresonding error (bottom).

Figure 4.8 shows the temperature profile in the coating for both the transient and steady case, for three metal temperature values. The temperature profiles of the transient and steady case are very close and the differences are not visible in the figure. Differences can be seen in the spatial temperature derivative $\left(\frac{d T}{d r}\right)$ values. The absolute values of the derivative at the metal-coating interface is higher in the steady case for all metal temperature values causing 
higher amount of heat transfer from the metal compared to the transient case. The difference in the derivative values between the two cases decrease as the metal temperature approaches liquid temperature. This explains the characteristics of heat transfer in the transient and steady case shown in Figure 4.8.
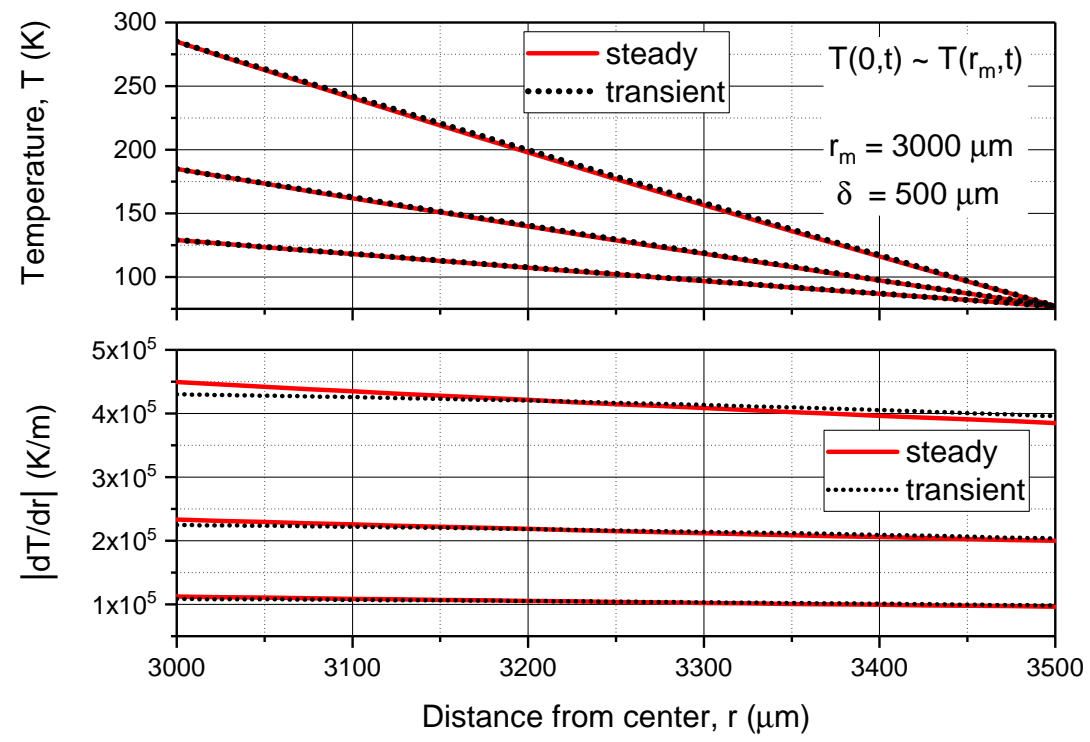

Figure (4.8) Temperature profile in the coating predicted using steady and transient case for various metal temperatures (top) and the spatial temperature derivative $\left(\frac{d T}{d r}\right)$ (bottom)

To conclude, during the late regime, the heat transfer values calculated by neglecting the heat capacity in the coating, considering the largest coating thickness of $500 \mu \mathrm{m}$, will result in about $4 \%$ error. This margin of error is acceptable in an engineering application. The error margin during the early regime is higher, however it will not effect the total cool-down time calculations, as the thermal diffusion time ( $t_{\text {early }} \approx \frac{\delta^{2}}{\alpha_{c}} \approx 1.5 \mathrm{~s}$ ) for the coating material is very low compare to the metal cool-down time of approximately $30 \mathrm{~s}$ for coating thickness of $500 \mu \mathrm{m}$. To apply the results discussed in this paper for a generic cryogenic cooling application, the following procedure is recommended. The critical heat flux $Q_{C H F}$ and the temperature at the critical heat flux $T_{C H F}$ for a particular cryogenic fluid for a bare surface must be known, 
which is documented for most engineering surfacers. If the intial temperature is $T_{i}$, then the coating thickness is, $\delta=\left(T_{i}-T_{C H G}\right) \frac{\lambda A}{Q_{C H F}}$, where $\lambda$ is the thermal conductivity of the coating. This coating thickness is an estimate that is within $10 \%$ to the fastest cooldown thickness value.

\subsection{Conclusions}

We succeeded in developing a model to a classical cryogenic observation, where insulated metals are shown to cooldown faster than their bare counterparts. We performed a systematic set of quenching experiments using copper cylinders coated with different thickness of low conductive epoxy coatings in both saturated and sub-cooled liquid nitrogen. From these experiments we noticed particularly, two important observations. First, a fairly constant heat transfer coefficent value in the nucleate regime for coated cylinders. Second, the critical heat flux value does not change with the coating thickness. In contrast, to earlier attempts in the literature to employ fitting parameters to model the film and transition regime, we approached this problem from a different perspective. We showed that the coating thickness, whose thermal resistance will bring the critical heat flux to the initial metal temperature, is close to less than ten percent of the optimum coating for the fast cool down. This hypothesis is validated with experimental data for several sub-cooling temperatures. This approach will give cryogenic application engineers a predictive tool to design systems for fast cool down by quenching in cryogenic liquid baths.

\section{Appendix - II}

\section{Mathematical description of the composite cylinder model:}

The model is presented for the case, where a metal cylinder of radius, $r_{m}$, is in perfect thermal contact with the coating material of constant thickness, $\delta$, along its length, $L$ and the coating-liquid interface is subjected to a limited value of heat transfer coefficient, $h$, with liquid nitrogen. The schematic of coated cylinder in liquid nitrogen pool is shown in the Figure 4.9. The heat diffusion equation for the metal and coating is given by,

$$
\frac{\alpha_{m}}{r} \frac{\partial}{\partial r}\left(r \frac{\partial \theta_{m}}{\partial r}\right)=\frac{\partial \theta_{m}(r, t)}{\partial t} \quad \text { in } \quad 0 \leq r<r_{m}, \quad t>0
$$




$$
\frac{\alpha_{c}}{r} \frac{\partial}{\partial r}\left(r \frac{\partial \theta_{c}}{\partial r}\right)=\frac{\partial \theta_{c}(r, t)}{\partial t} \quad \text { in } \quad r_{m} \leq r<\left(r_{m}+\delta\right), \quad t>0
$$

in this, $\theta=T-T_{f}$, is the wall superheat. The cylinder is subjected to the following initial and boundary conditions,

$$
\begin{aligned}
\theta_{m}(r, t=0) & =\theta_{c}(r, t=0)=T_{i} \\
\theta_{m}(r \rightarrow 0, t) & \Rightarrow \text { finite } \\
\theta_{m}\left(r=r_{m}, t\right) & =\theta_{c}\left(r=r_{m}, t\right) \\
\left.\lambda_{m} \frac{\partial \theta_{m}}{\partial r}\right|_{r=r_{m}} & =\left.\lambda_{c} \frac{\partial \theta_{c}}{\partial r}\right|_{r=r_{m}} \\
-\left.\lambda_{c} \frac{\partial \theta_{c}}{\partial r}\right|_{r=r_{m}+\delta} & =\left.h \theta_{c}\right|_{r=r_{m}+\delta}
\end{aligned}
$$

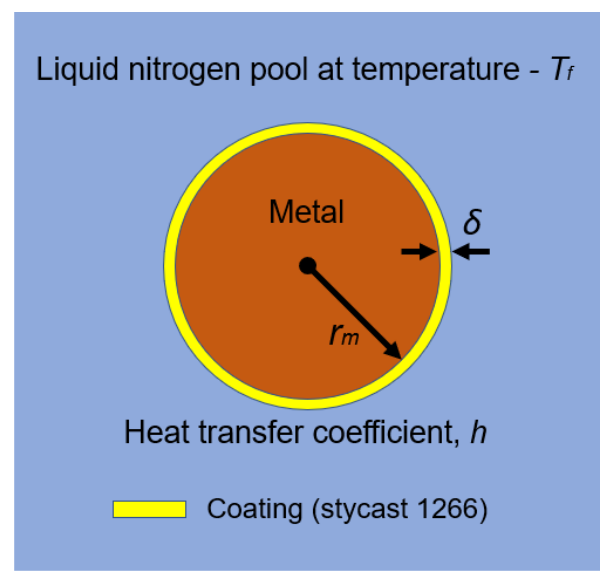

Figure (4.9) Schematic of the cross-section of a coated cylinder with perfect thermal contact at the metal-coating interface and limited value of heat transfer coefficient, $h$, with liquid nitrogen.

Using initial and boundary conditions to solve Equation 4.6 and Equation 4.7. The solution for the temperature variation in metal and coating is given by [57], 


$$
\begin{aligned}
& \theta_{m}(r, t)=\sum_{n=1}^{\infty} \frac{T_{i}}{N_{n}} e^{-\beta_{n}^{2} t} \psi_{1 n}(r)\left[\frac{\lambda_{m}}{\alpha_{m}} \int_{r^{\prime}=0}^{r_{m}} r^{\prime} \psi_{1 n}\left(r^{\prime}\right) d r^{\prime}+\frac{\lambda_{c}}{\alpha_{c}} \int_{r^{\prime}=r_{m}}^{r_{m}+\delta} r^{\prime} \psi_{2 n}\left(r^{\prime}\right) d r^{\prime}\right] \\
& \theta_{c}(r, t)=\sum_{n=1}^{\infty} \frac{T_{i}}{N_{n}} e^{-\beta_{n}^{2} t} \psi_{2 n}(r)\left[\frac{\lambda_{m}}{\alpha_{m}} \int_{r^{\prime}=0}^{r_{m}} r^{\prime} \psi_{1 n}\left(r^{\prime}\right) d r^{\prime}+\frac{\lambda_{c}}{\alpha_{c}} \int_{r^{\prime}=r_{m}}^{r_{m}+\delta} r^{\prime} \psi_{2 n}\left(r^{\prime}\right) d r^{\prime}\right]
\end{aligned}
$$

in this, the eigen values $\left(\beta_{n}\right)$ are the roots of the following transcendental equation:

$$
\left|\begin{array}{ccc}
J_{0}(\gamma) & -J_{0}\left(\frac{r_{m} \eta}{r_{m}+\delta}\right) & -Y_{0}\left(\frac{r_{m} \eta}{r_{m}+\delta}\right) \\
K J_{1}(\gamma) & -J_{1}\left(\frac{r_{m} \eta}{r_{m}+\delta}\right) & -Y_{1}\left(\frac{r_{m} \eta}{r_{m}+\delta}\right) \\
0 & \frac{H}{\eta} J_{0}(\eta)-J_{1}(\eta) & \frac{H}{\eta} Y_{0}(\eta)-Y_{1}(\eta)
\end{array}\right|=0
$$

where, $J_{0}, Y_{0}$ and $J_{1}, Y_{1}$ are the Bessel functions of order 0 and 1 respectively and the values of $\gamma, \eta, H$ and $K$ are given by,

$$
\gamma=\frac{r_{m} \beta_{n}}{\sqrt{\alpha_{c}}} ; \quad \eta=\frac{\left(r_{m}+\delta\right) \beta_{n}}{\sqrt{\alpha_{c}}} ; \quad H=\frac{\left(r_{m}+\delta\right) h}{\sqrt{\lambda_{c}}} ; \quad K=\frac{\lambda_{m}}{\lambda_{c}} \frac{\alpha_{m}}{\alpha_{c}}
$$

After calculating the eigen values $\left(\beta_{n}\right)$, the temperature variation inside the metal and coating material is calculated using Equation 4.8 and Equation 4.9, in which $N_{n}, \psi_{1 n}(r)$ and $\psi_{2 n}(r)$ are given by, 


$$
\begin{aligned}
N_{n} & =\frac{\lambda_{m}}{\alpha_{m}} \int_{r^{\prime}=0}^{r_{m}} r^{\prime} \psi_{1 n}^{2}\left(r^{\prime}\right) d r^{\prime}+\frac{\lambda_{c}}{\alpha_{c}} \int_{r^{\prime}=r_{m}}^{r_{m}+\delta} r^{\prime} \psi_{2 n}^{2}\left(r^{\prime}\right) d r^{\prime} \\
\psi_{1 n}(r) & =J_{0}\left(\frac{\beta_{n} r}{\sqrt{\alpha_{m}}}\right) \\
\psi_{2 n}(r) & =A_{2 n} J_{0}\left(\frac{\beta_{n} r}{\sqrt{\alpha_{m}}}\right)+B_{2 n} Y_{0}\left(\frac{\beta_{n} r}{\sqrt{\alpha_{c}}}\right)
\end{aligned}
$$

where,

$$
\begin{aligned}
A_{2 n} & =\frac{1}{\Delta}\left[J_{0}(\gamma) Y_{1}\left(\frac{r_{m} \eta}{r_{m}+\delta}\right)-K J_{1}(\gamma) Y_{0}\left(\frac{r_{m} \eta}{r_{m}+\delta}\right)\right] \\
B_{2 n} & =\frac{1}{\Delta}\left[K J_{1}(\gamma) J_{0}\left(\frac{r_{m} \eta}{r_{m}+\delta}\right)-J_{0}(\gamma) J_{1}\left(\frac{r_{m} \eta}{r_{m}+\delta}\right)\right] \\
\Delta & =J_{0}\left(\frac{r_{m} \eta}{r_{m}+\delta}\right) Y_{1}\left(\frac{r_{m} \eta}{r_{m}+\delta}\right)-J_{1}\left(\frac{r_{m} \eta}{r_{m}+\delta}\right) Y_{0}\left(\frac{r_{m} \eta}{r_{m}+\delta}\right)
\end{aligned}
$$




\section{Section - II}

\section{Development of a tissue snap-freezer without sacrificial cryogens}


CHAPTER 5

\title{
Tissue snap-freezer: design ${ }^{12}$
}

\begin{abstract}
This chapter describes the design process of an electrically powered tissue snap-freezer, which is aimed to provide an active cooling alternative to the conventional passive cooling methods, where liquid coolants releasing vapor cryogens into the atmosphere are usually used to snap-freeze the samples. The conventional procedure is not user friendly and is laborious as reducing the lag time from excision time to freezing depends on the logistic organizational structure within a hospital. Moreover snap-freezing must be done as soon as possible after tissue excision to preserve the tissue quality for molecular tests [16]. The developed snap-freezing device can be used directly at the location where the tissue is acquired and therefore helps in reducing the time lag between the acquisition and freezing. This device also provides variable cooling rates and thus facilitates the study of the effect of freezing conditions on the various molecular processes in the samples. This chapter also describes the development of a heat transfer model used to predict the cryo-vial cooldown in the snap-freezer.
\end{abstract}

\subsection{Systematic design}

In this section, the design process of the apparatus is discussed. To reduce the lag-time between the tissue acquisition and freezing, the device must allow tissue processing at the location where the biopsy is performed. In addition, the apparatus has additional features to facilitate research on the impact of freezing characteristics on the tissue viability. The requirements for the snap-

\footnotetext{
${ }^{1}$ Published as : Vanapalli, S., Jagga, S., Holland, H. and ter Brake, M., 2017, December. A tissue snap-freezing apparatus without sacrificial cryogens. In IOP Conference Series: Materials Science and Engineering (Vol. 278, No. 1, p. 012126). IOP Publishing.

${ }^{2}$ Published as : van Limbeek, M.A., Jagga, S., Holland, H., Ledeboer, K., ter Brake, M. and Vanapalli, S., 2019. Cooling of a vial in a snapfreezing device without using sacrificial cryogens. Scientific reports, 9(1), pp.1-9.
} 
freezer mentioned below are finalized after discussing with the UMC Amsterdam, which is research enter for cancer diagnosis and is also the medical research partner of the project CryoOn.

- Adjustable cold sink temperature between 80-200 K; the lower limit of $80 \mathrm{~K}$ is chosen for the liquid nitrogen based snap-freezing procedures and the upper range of $200 \mathrm{~K}$ is for the dry-ice based snap-freezing procedures [13].

- Cooling rate may be varied with a minimum value lower than the liquid nitrogen quenching rate; the average cooling rate for the aluminum vial in liquid nitrogen is about $-25 \mathrm{~K} / \mathrm{s}$ for the temperature values above 120 $\mathrm{K}$.

- No use of dangerous substances and limited venting of gases in line with the safety norms of the operation theatre.

- Standard cryo-vials should be used; the device should be designed to use the cryo-vials used by the UMC Amsterdam for tissue snap-freezing.

- The device should be able to snap-freeze six vials in a row. During discussion with our project partner with UMC Amsterdam, multiple samples upto six are taken from a single patent and are expected to be frozen at the same time.

- Only utility available is electricity and small canister of gas is allowed. The device should be cooled using electrically operated coolers, as no liquid cryogens are allowed due to the safety regulations inside the operation theater.

\subsubsection{Cooling concepts}

The snap-freezing device is bound to operate on electricity as no cryogenic coolants are allowed to use. Keeping this in mind and to build a compact device, a pulse tube cryo-cooler is chosen to provide the cold. The heat from vial to the cold surface of the cryocooler can be transported by different means: a direct contact between both the surfaces, a cold liquid or gas as a heat transport medium between the two surfaces.

Direct contact: In this procedure, a vial greased with a thin layer of good conducting material to bridge the surface roughness gap, can directly be brought 
in contact with a cold surface. The advantage of this method is a good thermal contact can be achieved for perfectly aligned surfaces; however for most practical surfaces some manufacturing defects are usually present, which can create hindrance to the heat transfer. Another limitation of this method is the low availability of the heat transfer area. Since the vials used at VUMC are cylindrical in shape, the maximum area of contact can be achieved by placing its flat bottom surface in contact with the cold surface. The aluminum vial used at the UMC Amsterdam for snap-freezing of the biopsied tissue has an external diameter $\left(D_{v}\right)$ of $14.5 \mathrm{~mm}$, a height $\left(H_{v}\right)$ of $25.0 \mathrm{~mm}$ and weighs $\left(m_{v}\right)$ around $1.4 \mathrm{~g}$, the bottom are of this vial is only $10 \%$ of the total area of the vial.

Liquid as a heat transport medium: In this approach, the vial can be dipped in a small liquid pool pre-cooled in a cold block attached to the cry-cooler. The cold block can be designed in a way that a small gap is created between the vial and the cold block, which upon immersion will be filled with the liquid covering the vial from three sides. The liquid in the gap will then acts as a medium of heat transfer between both the surfaces. For a sub-millimeter gap size the heat transfer is majorly due to thermal diffusion, in which heat transfer rate is proportional to the thermal conductivity of the liquid and is inversely proportional to the gap size, i.e. different cooling rates can be achieved by varying these two parameters. Other advantages of this method is that a good thermal contact and a greater heat transfer area can be achieved.

However, to meet the purpose, liquid in the gap must stay in its sub-cooled state during the heat transfer process. Table 5.1 shows the liquids with boiling point above room temperature $(\approx 295 \mathrm{~K})$ and freezing point in the desired temperature range of $80 \mathrm{~K}$ to $200 \mathrm{~K}$. Isopentane covers majority of the desired temperature range and therefore can be considered as the best available option. A 10 micron layer of isopentane between the vial and the cold surface provides a heat transfer rate of about $1.7 \mathrm{Wcm}^{-2} \mathrm{~K}^{-1}$. The disadvantage of this approach is that achieving end temperature values below isopentane freezing point of $113 \mathrm{~K}$ is not possible. Also isopentane is an extremely flammable liquid and considered as toxic material [69] and therefore is not allowed to use inside the operation theater. 


\begin{tabular}{l|l|l}
\hline Substance & $\begin{array}{l}\text { Freezing point at } \mathbf{1} \\
\text { atm }[\mathbf{K}]\end{array}$ & $\begin{array}{l}\text { Boiling point at 1 } \\
\text { atm }[\mathbf{K}]\end{array}$ \\
\hline \hline Isopentane & 113 & 301 \\
\hline Novec 7200 & 135 & 349 \\
\hline Novec 7100 & 138 & 334 \\
\hline Novec 7000 & 151 & 304 \\
\hline Ethanol & 159 & 351 \\
\hline
\end{tabular}

Table (5.1) Liquids with boiling point above room temperature and freezing point in the desired temperature range of $80 \mathrm{~K}$ to $200 \mathrm{~K}$.

Gas as a heat transport medium: In this approach a non-condensible gas can be used to transport the heat from the vial. This methodology offers all the advantages possible with using the liquid as a coolant i.e., maximum surface contact and variable cooling rate. Additionally, it also allows us to cover the entire temperature range of $80 \mathrm{~K}$ to $200 \mathrm{~K}$. To avoid venting the gas into the atmosphere, a closed circuit for the gas-flow can be used. A small and portable gas canister would serve the purpose of gas supply.

To reduce the snap-freezer footprint, an internal forced convective flow in the gap between the vial and a cold surface, is chosen as the forced convective cooling principle. The advantage of using approach is that the heat transfer can be enhanced by selecting for a sub-mm gas-gap size for which thermal diffusion from the vial to the cold surface through the gas-gap becomes significant. The heat transfer rate due to thermal diffusion is proportional to the thermal conductivity of the liquid and is inversely proportional to the gap size, i.e. different cooling rates can be achieved by varying these two parameters. The heat transfer using thermal diffusion is also used in a gas-gap based heat switch for cryogenic applications [70-72].

Now, a further assessment is done to estimate the achievable cooling rates using this approach. To do this, a worst case scenario is considered where no gas flow is considered in the gap i.e. a static gas is present between the vial and the cold surface. A commercial aluminum vial with the dimensions discussed above is used for the analysis. The heat transfer is assumed to occur through the side area of the cylindrical shaped vial, which for the vial used is the roughly $80 \%$ of the total heat transfer area. For estimation, the aluminum 
vial is assumed as a lumped mass, this assumption is valid and a detailed discussion is added later in the section 5.3. The heat transfer in the sub millimeter gas-gap size is assumed majorly due to thermal diffusion only and is given by,

$$
-m_{v} c_{v}(T) \frac{d T}{d t}=\bar{\lambda}_{\mathrm{g}}(T) A \frac{T-T_{\infty}}{d}
$$

In this $m_{v}, c_{v}$ and $T$ is the mass, specific heat capacity and temperature of the vial respectively. $\overline{\lambda_{\mathrm{g}}}$ is the temperature averaged thermal conductivity of the gas in the gas-gap. $d$ is the thickness of the gas-gap, $A$ is the heat transfer area of the vial and $T_{\infty}$ is the temperature of the cold mass. The temperature dependent thermal properties of the aluminum material are shown in the Appendix. Using Equation 5.1, the calculated temperature variation for the helium gas in different gas-gap sizes are shown in the Figure 5.1. It can be seen that for gas-gap size values lower than $0.6 \mathrm{~mm}$, a lower values of cooldown time compare to the liquid nitrogen can be achieved. This size of the gas-gap is possible using the existing manufacturing techniques.

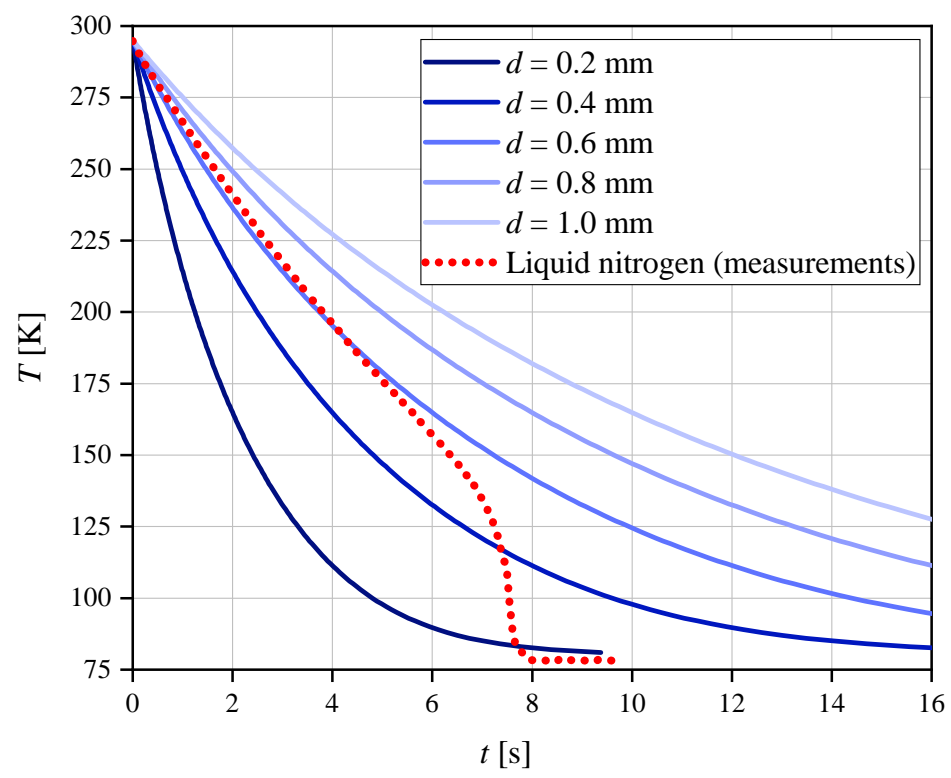

Figure (5.1) Estimated cool-down time values in a gas-gap for various gap sizes and its comparison with the measurements in liquid nitrogen. 
One should also keep in mind that the cooling speed of the vial can also be varied by using a forced convective flow of the gas around the vial. When a gap size of sub-mm is used, the flow in the gap can be considered to be in the laminar flow regime. The enthalpy carried by the gas flow is then proportional to the mass flow rate allowing variable cooling rate of the cryo-vial.

To summarize, following the assessment of the discussed cooling concepts, the cryo-vial for tissue snap-freezing over the desired temperature range of 80 $\mathrm{K}$ to $200 \mathrm{~K}$ can be best cooled by using the cold gas as a heat transfer medium and therefore is chosen as the cooling principle for the designed apparatus.

\subsection{Design methodology}

After finalizing the cooling principle, the main objectives of the design process is the system compactness (size) and the energy efficiency. In this section, a design methodology for various functional components of the device is discussed to meet these objectives. Figure 5.2 shows the schematic of the snap-freezer depicting its main components and their functional description.

Cryo-vial: The snap-freezer is designed for a commercial cryo-vial made of aluminium with the dimensions discussed above. This vial is used at the UMC Amsterdam for snap-freezing of the biopsied tissue. When the vial is inserted into the snap-freezing device, the gap $d$ between the external vial wall and the cold thermal reservoir (TESU) is $0.4 \mathrm{~mm}$. This gap size is chosen from the estimated temperature values shown in the Figure 5.1. For which cooling rate higher than the liquid nitrogen can be achieved for the temperature values greater than $120 \mathrm{~K}$.

Cold gas: The working gases used in the gas-gap act as a medium of heat transfer from the vial to the cold thermal buffer (TESU). The working gases used in the current snap-freezer are helium, nitrogen and heliox. Heliox is a mixture of $79 \%$ helium and $21 \%$ oxygen gas. It is a medically approved gas for ventilation of infants in hospitals having breathing issues. The motivation of using this gas mixture is the possible quick availability and adoption in a medical environment.

The data of helium and nitrogen is obtained from [73], whereas little is documented about the properties of Heliox. We therefore use the model of Mason and Saxena [74] to describe the thermal conductivity and its temperature de- 
pendency, where they extended the empirical model of Wassiljewa [75]. Here, the conductivity of a mixture is given by,

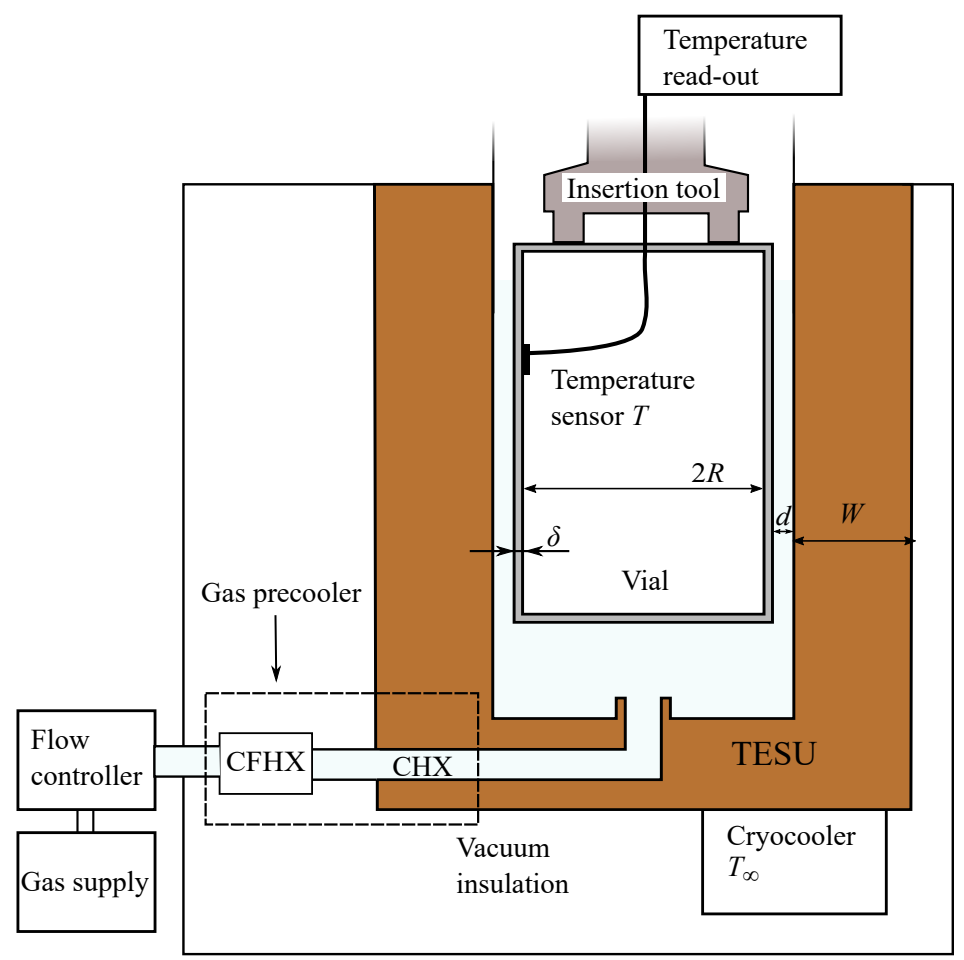

\begin{tabular}{l|l}
\hline Component & Function \\
\hline \hline Cryo-vial & Holds the tissue \\
\hline Cold gas & Removes heat from the cryo-vial \\
\hline Thermal Energy Storage Unit (TESU) & A cold buffer \\
\hline Cryocooler & Generates cooling \\
\hline Gas pre-cooler & $\begin{array}{l}\text { Pre-cool the warm gas flow and cools } \\
\text { the gas flow to a set low temperature of } \\
\text { the TESU }\end{array}$ \\
\hline Gas supply and flow controller & Controls gas-flow in the apparatus \\
\hline Vacuum Insulation & $\begin{array}{l}\text { Reduce parasitic heat leak to the cold } \\
\text { environment }\end{array}$ \\
\hline
\end{tabular}

Figure (5.2) Conceptual design of the apparatus showing the main functional components and their functional description. 


$$
k_{\mathrm{m}}=\sum_{i=1}^{n} \frac{y_{i} k_{i}}{\sum_{j=1}^{n} y_{j} A_{i, j}} .
$$

$i$ and $j$ denote the components in the mixture, with their molar fraction y. $A_{i, j}$ is a function, yet to be determined. Equation 5.2 reduces for a two component system to:

$$
k_{\mathrm{m}}=\frac{y_{\mathrm{He}} k_{\mathrm{He}}}{y_{\mathrm{He}}+y_{\mathrm{Ox}} A_{1,2}}+\frac{y_{\mathrm{Ox}} k_{\mathrm{Ox}}}{y_{\mathrm{He}} A_{2,1}+y_{\mathrm{Ox}}},
$$

where the subscripts $\mathrm{He}$ and $\mathrm{Ox}$ refer to the helium and oxygen components. Here $A_{1,1}=A_{2,2}=1$. The interaction between the helium and oxygen gas however are modelled as follows:

$$
\begin{aligned}
& A(1,2)=\frac{e\left[1+\left(k_{\mathrm{He}} / k_{\mathrm{Ox}}\right)^{1 / 2} \cdot\left(M_{\mathrm{He}} / M_{\mathrm{Ox}}\right)^{1 / 4}\right]^{2}}{\left[8\left(1+M_{\mathrm{He}} / M_{\mathrm{Ox}}\right)\right]^{1 / 2}}, \\
& A(2,1)=\frac{e\left[1+\left(k_{\mathrm{Ox}} / k_{\mathrm{He}}\right)^{1 / 2} \cdot\left(M_{\mathrm{Ox}} / M_{\mathrm{He}}\right)^{1 / 4}\right]^{2}}{\left[8\left(1+M_{\mathrm{Ox}} / M_{\mathrm{He}}\right)\right]^{1 / 2}},
\end{aligned}
$$

in which $M$ is the molar mass and $e=1$. Equation 5.2 was then evaluated and fitted to obtain a linear relation $k_{\mathrm{m}}=a_{\mathrm{m}}+b_{\mathrm{m}} T$, used in solving Equation 5.7. We found $a_{\mathrm{m}}=18.70 \times 10^{-3} \mathrm{~W} \mathrm{~m}^{-1} \mathrm{~K}^{-1}$ and $b_{\mathrm{m}}=0.3287 \times$ $10^{-3} \mathrm{Wm}^{-1} \mathrm{~K}^{-2}$. The thermal conductivity, specific heat capacity, density and viscosity of these gases over the temperature range of interest i.e. between $300 \mathrm{~K}$ to $80 \mathrm{~K}$ are shown in Appendix.

Thermal Energy Storage Unit (TESU): It is essentially a large heat capacity used to bridge the gap between the cold energy demand and the cold supply with a cryocooler. Without a TESU, a large power cyrocooler is required increasing the overall size of the apparatus. An estimation of the required cooling power without a TESU clarifies this hypothesis. The cooling energy required is equal to the sum of the energy content in the tissue and the cryovial between $295 \mathrm{~K}$ to $77 \mathrm{~K}$. The tissue may be assumed to be made of only water with a latent heat of liquid to ice transformation at $273 \mathrm{~K}$. The thermo-physical properties of the tissue and the cryo-vial are shown in Table 5.2. For a typical sized tissue of about $20 \mathrm{mg}$, the energy content is about 
$16 \mathrm{~J}$ and that of the commercial aluminum cryo-vial that weighs $1.4 \mathrm{~g}$ is about $220 \mathrm{~J}$. Therefore an average cooling power of $23.6 \mathrm{~W}$ in the cooling time of 10 seconds is required to cool only the tissue and the cryo-vial, without considering the parasitic heat leaks to the environment. A commercial cryocooler that satisfies this requirement weighs $5.6 \mathrm{~kg}$ and occupies $0.25 \mathrm{~m}^{3}$. A thermal buffer mitigates the cold energy supply and demand gap, and reduces the size of the cryocooler.

The TESU used is made up of copper material. Considering design margins and a temperature tolerance of $1 \mathrm{~K}$, a copper mass of $1.25 \mathrm{~kg}$ with an energy content of about $240 \mathrm{~J}$ at $77 \mathrm{~K}$, is sufficient to provide the desired cooling capacity for a tissue enclosed in a vial. For temperature dependent thermal properties of the copper material, see the Appendix. By using a TESU, a much smaller power cryo-cooler is needed to prepare the apparatus for snapfreezing. The cryo-cooler used to cool the TESU provides around $3.7 \mathrm{~W}$ of cooling power at $77 \mathrm{~K}$, i.e. after each snap-freezing operation, the cryocooler is able to remove the heat added to the TESU $(\approx 236 \mathrm{~J})$ in roughly one minute and thus allows another snap-freezing operation. The details of the cryo-cooler used are discussed next.

\begin{tabular}{l|l|l|l}
\hline & $\begin{array}{l}\text { Biological tissue } \\
\text { (water) }\end{array}$ & Vial (Aluminum) & $\begin{array}{l}\text { TESU } \\
(\text { Copper) }\end{array}$ \\
\hline \hline Density & $1 \mathrm{~g} / \mathrm{cm}^{3}$ & $2.7 \mathrm{~g} / \mathrm{cm}^{3}$ & $8.96 \mathrm{~g} / \mathrm{cm}^{3}$ \\
\hline Heat capacity & $4 \mathrm{~J} / \mathrm{g} \cdot \mathrm{K}(\mathrm{T}>273 \mathrm{~K})$ & $0.91 \mathrm{~J} / \mathrm{g} \cdot \mathrm{K}(\mathrm{T}=300 \mathrm{~K})$ & $0.19 \mathrm{~J} / \mathrm{g} \cdot \mathrm{K}$ \\
& $334 \mathrm{~J} / \mathrm{g}(\mathrm{T}=273 \mathrm{~K})$ & $0.36 \mathrm{~J} / \mathrm{g} \cdot \mathrm{K}(\mathrm{T}=77 \mathrm{~K})$ & $(\mathrm{T}=77 \mathrm{~K})$ \\
& $2 \mathrm{~J} / \mathrm{g} \cdot \mathrm{K}(\mathrm{T}<273 \mathrm{~K})$ & & \\
\hline
\end{tabular}

Table (5.2) Material properties of the biopsy tissue sample, cryo-vial and the TESU. See Appendix for the temperature dependent thermal properties of aluminum and copper material.

Cryocooler: The cryo-cooler used in the snap-freezer is LPT9310, which is a commercial cryo-cooler manufactured by Thales Cryogenics. The calibrated values of steady-state and transient cooling power are shown in the Figure 5.3a. A detailed discussion on the cooling power calculation methodology is presented in Chapter 7. The size of the cryo-cooler is chosen to keep the preparation time of roughly two hours. Figure 5.3b shows the cool-down curve of the TESU used attached to the cold-end of the cryo-cooler. The temperature of cryo-cooler's cold head can be set to different temperature values 
and is determined by the reading voltage of a calibrated diode.

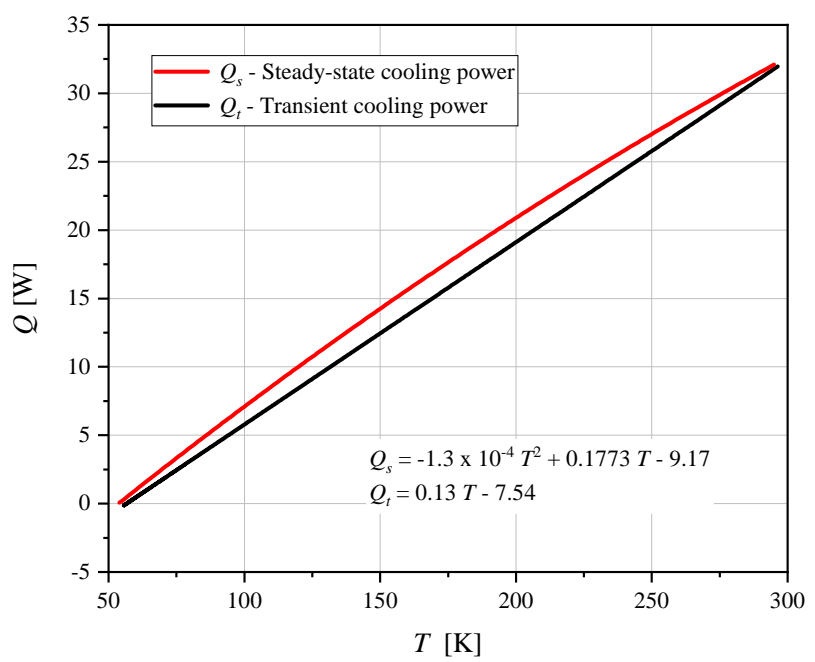

(a)

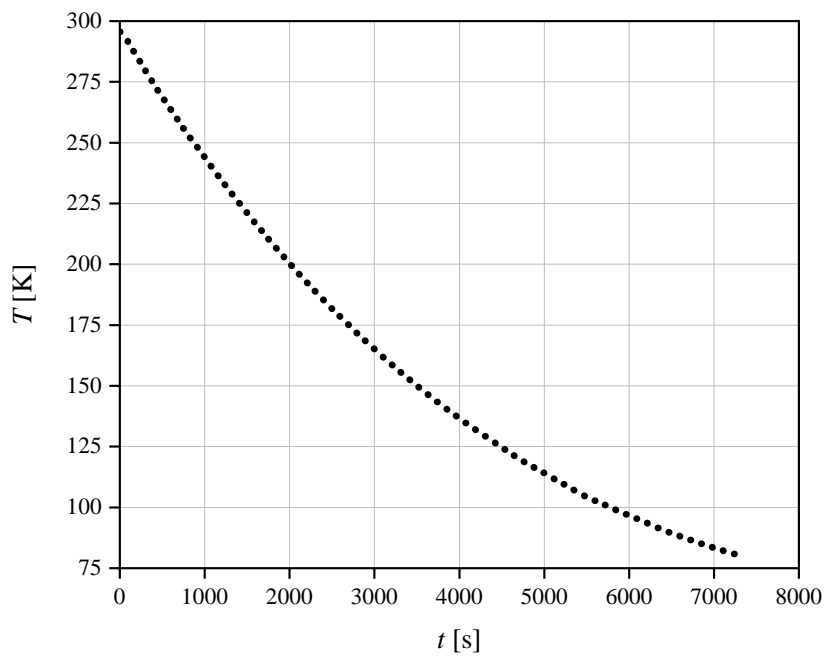

(b)

Figure (5.3) (a) - Calculated steady-state and transient cooling power values of the cryo-cooler for different cold-end temperature values. (b) - Cool-down curve of the TESU (copper mass of $1.25 \mathrm{~kg}$ ) attached to the cold-end of the cryo-cooler. 
Gas supply and flow controller: A small bottle of pressurized helium gas is used for the gas supply, which is then connected to a controller that controls the gas-flow into the apparatus.

Gas pre-cooler: The gas pre-cooler consist of two components - Counter Flow Heat Exchanger (CFHX) and the cold heat exchanger (CHX). The CFHX serves two purposes; first it improves the energy efficiency of the apparatus, and second the outgoing gas from the TESU should be heated to the ambient temperature as the flow instrumentation, and the circulator are specified to operate at room temperature. The thermal energy content of a $40 \mathrm{mg} . \mathrm{s}^{-1}$ flow of helium gas between $300 \mathrm{~K}$ and $77 \mathrm{~K}$ is about $45 \mathrm{~W}$. Without a CFHX all this energy should be supplied to the gas by the TESU. A counter flow heat exchanger with an efficiency of about $95 \%$ will deposit about $22.5 \mathrm{~W}$ and reduces the cooling power requirement by approximately $50 \%$. Therefore, reducing the required size of the TESU. The incoming gas after passing through the CHFX, enters into the cold heat exchanger (CHX), which is designed to cool the gas flow to a set temperature of the TESU.

Insulation: To reduce heat leaks to the TESU and the heat exchangers, these components are placed inside a vacuum chamber with the vacuum level maintained lower than $10^{-6} \mathrm{~Pa}$.

To summarize, the current snap-freezer is designed to cool the commercial cryo-vials made up of aluminum. A variable cooling rate for the enclosed tissue can be achieved by varying the gas thermal properties, gas flow rate and the TESU temperature. The snap-freezer vents low quantity of helium gas or a mixture of medical approved helium, oxygen mixture. From the operational point of view the following phases are recognized. The preparation phase in which the cryo-cooler is turned on to cool the TESU and later a low flow rate of about $4 \mathrm{mgs}^{-1}$ helium gas of about is circulated to cool the counter flow heat exchanger, takes about two hours. The cooling phase where a cryo-vial is inserted in the apparatus for snap-freezing, takes less than a minute. The regeneration phase, in which the cryocooler cools the TESU to the original setting, which takes about one minute when the apparatus is once again ready for the next cryo-vial insertion. 


\subsection{Model}

In this section, a heat transfer model for thermal interaction of the cryo-vial and the heat sink via the gas-gap is presented. To simplify the model, following assumptions are made.

\section{Assumptions}

(i) One dimensional domain is considered for the heat transfer in the gasgap

The thermal diffusion time scale in a domain of length $L$ is, $t_{d} \propto L^{2}$. The aspect ratio of the gas-gap, i.e. the ratio of the height to the width of the gasgap, $H_{v} / d \approx 60$, i.e. the diffusion time scale along the longitudinal direction is very high compared to the radial direction and therefore heat transfer in the longitudinal direction can be neglected compared to the radial direction.

\section{(ii) Radial effects in the gas-gap are neglected}

The ratio of the vial diameter to the gas-gap width, $D_{v} / d \approx 35$, which is large enough to neglect the curvature effect in the gas-gap. Therefore, heat transfer in the gas-gap is assumed as the heat transfer between two parallel plates.

\section{(iii) Flow regime around the vial is laminar and fully developed}

Reynolds number is used to classify the fluid flow regime as laminar or turbulent. The nature of the flow (laminar vs. turbulent) not only depends on its velocity but also on the density, viscosity, and the corresponding length scale. The Reynolds number for the gas-gap is $R e_{\mathrm{g}}=\rho_{\mathrm{g}} v_{\mathrm{g}} d / \mu_{\mathrm{g}}$. For flow between parallel plates, the flow is laminar when $R e_{\mathrm{g}}<1400$. Figure 5.4 shows the calculated value of Reynolds number $(R e)$ as a function of helium gas mass flow rate. It can be seen that for a flow rate up to $40 \mathrm{mg} . \mathrm{s}^{-1}$, which is the maximum design value for helium gas, $R e_{\mathrm{g}}<<1400$ i.e. flow rate will be in the laminar regime.

The thermal entrance length, $\ell(U)$, which is the measure of development of the thermal boundary layer during the flow is given by [45], $\ell(U)=0.05 d\left(\operatorname{Re}_{\mathrm{g}} P r_{\mathrm{g}}.\right)$, in this $P r_{\mathrm{g}}$ is the Prandtl number. The calculated thermal entrance length values for several flow rates are also shown in Figure 5.4. For a flow rate up to 40 $\mathrm{mg} . \mathrm{s}^{-1}$, thermal entrance length is less than $6 \%$ of the vial length, therefore 
flow may be assumed as fully developed inside the gas-gap.
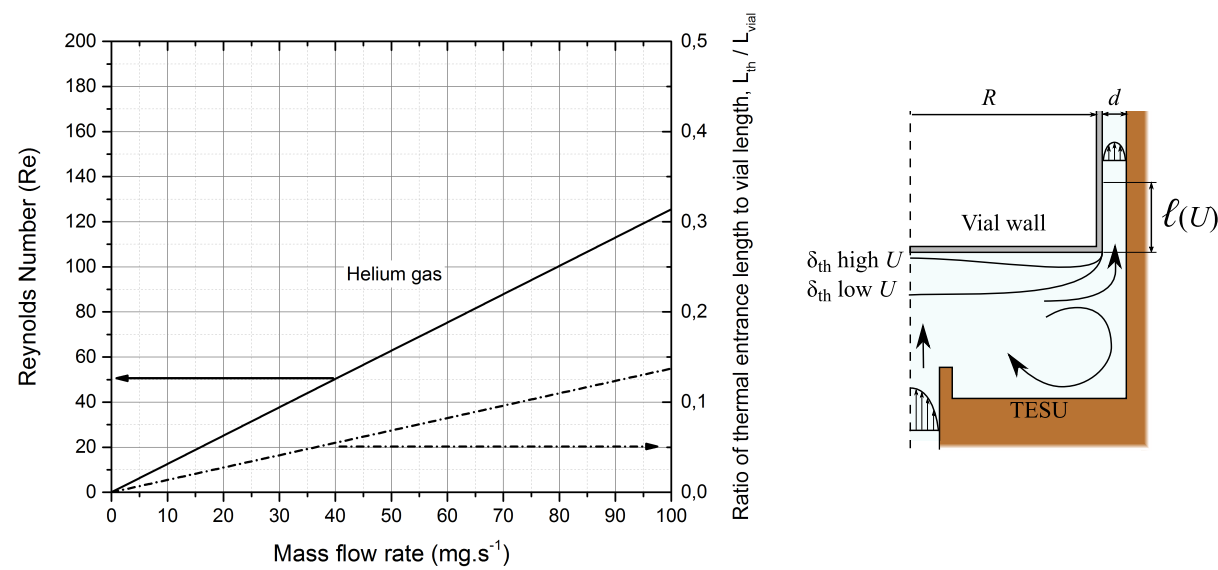

Figure (5.4) Reynolds number and thermal entrance length for a flow of helium gas in the gas-gap as a function of mass flow rate. In the sketch, the gas-jet is impacting the bottom of the vial and subsequently entering the gasgap, where arrows indicate the main flow characteristics.

\section{(iv) Temporal effects in all three domains - vial, gas-gap and TESU are neglected}

Let us now first estimate the total energy change $\Delta E=m_{v} \int_{T_{\infty}}^{T_{\text {amb }}} c_{v}(T) \mathrm{d} T$ to cool down the vial from ambient temperature to the desired temperature of the TESU, $T_{\infty}$. Here $c_{v}$ is the vial specific heat and $m_{v}$ the mass of the vial. Since most mass is at the sidewall of the vial, we obtain $m_{v} \approx \rho_{v} A_{v} \delta$, where $A_{v}=\pi D_{v} H_{v}$ is the area of the vial wall. Balancing $\Delta E$ with the heat transferred across the gap, we obtain the cooling time scale of the vial: $t \sim$ $\left(\rho_{v} c_{v} A_{v} \delta\right) /\left(A_{v} \lambda_{\mathrm{g}} / d\right) \sim \mathscr{O}(1)$ second. Any convective heat transfer in the gap are of the order unity and alter this time scale by one order of magnitude at most. Using $t$, one can estimate the Fourier number, $\mathscr{F} o=\alpha t / \ell_{c}^{2}$, to determine whether or not temporal effects are important in the diffusion equation. Here $\ell_{c}$ and $\alpha$ are the characteristic length scale and thermal diffusivity of the vial, gas, and TESU, respectively. For the vial, $\ell_{c}$ is the wall thickness $\delta$, for the gas it is the gap thickness $d$ and for the TESU we use the wall thickness $W$. We find that the Fourier number is much larger than unity for all three domains, see Table 5.3. This implies that the temperature profiles inside the 
three domains adapt instantly with respect to the cooling time scale of the vial. As a result, the profile in the gap can be obtained by solving the steady non-linear heat equation in the gap, where the non-linearity is a consequence of the relative large variation of the thermal conductivity $\lambda_{\mathrm{g}}$ over the temperature range. One can represent the heat flux using $\bar{\lambda}_{\mathrm{g}}=1 / \Delta T \int \lambda_{\mathrm{g}}(T) \mathrm{d} T$ as the mean conductivity of the gas to obtain $q^{\prime \prime}=-\bar{\lambda}_{\mathrm{g}} \Delta T / d$. Here $d$ is the gap width and $\Delta T$ the temperature difference between the vial wall and the TESU wall.

\begin{tabular}{l|ccc} 
& $\ell_{c}[\mathrm{~m}]$ & $\alpha\left[\mathrm{m}^{2} \mathrm{~s}^{-1}\right]$ & $\tau[\mathrm{s}]$ \\
\hline vial wall $\delta$ & $2 \times 10^{-4}$ & $1 \times 10^{-4}$ & $4 \times 10^{-4}$ \\
gap $d$ & $4 \times 10^{-4}$ & $2 \times 10^{-5}$ & $8 \times 10^{-3}$ \\
TESU wall $W$ & $1 \times 10^{-2}$ & $1.5 \times 10^{-4}$ & $6 \times 10^{-1}$ \\
\hline
\end{tabular}

Table (5.3) Estimation of the characteristic lengthscale $\ell_{c}$, thermal diffusivity $\alpha$ and thermal timescale $\tau=\ell_{c}^{2} / \alpha$. Values evaluated at $100 \mathrm{~K}$, for a aluminium vial, helium filled gap and copper TESU.

\section{(iv) Cryo-vial and TESU as lumped capacitance}

A mass of given dimensions interacting thermally with its surroundings may be assumed as a lumped heat capacity (i.e. no temperature gradient inside the mass) when the following conditions are satisfied.

- Biot Number $(\mathscr{B} i)$, the ratio of internal thermal resistance to the thermal resistance at the surface of the mass is less than one, i.e. $\mathscr{B} i<<1$.

- Fourier number $(\mathscr{F} o)$, the ratio of thermal diffusive transport rate to the storage rate in the mass is greater than one, i.e. $\mathscr{F} o>1$.

We now estimate the Biot numbers of the vial and the TESU: $\mathscr{B} i=h \ell_{c} / \lambda$ , using the same characteristic length $\ell_{c}$ scales as before, $h$ is the heat transfer coefficient, which is for the diffusion across the gap is simply $\bar{\lambda}_{g} / d$ and $\lambda$ is the thermal conductivity of the vial and TESU, respectively. Evaluation of $\mathscr{B} i$ lets us discard the possibility of temperature gradients occurring inside the vial and the TESU as $\mathscr{B} i \ll 1$ for both cases.

For $t>\tau$, the Fourier number for the vial and the TESU will be greater than one. The values of $\tau$ for all three domains are mentioned in the Table 5.3, which are much less compared to the total cooling time of the vial $(\approx 10 \mathrm{~s})$. As a consequence we can treat vial and the TESU as a lumped capacitance. 
(vi) Temperature of the heat sink remains constant during the cooling of vial

As discussed before, for a typical sized tissue of about $20 \mathrm{mg}$, the energy content is about $16 \mathrm{~J}$ and that of the commercial aluminum cryo-vial weighs $1.4 \mathrm{~g}$ is about $220 \mathrm{~J}$. The TESU is made of copper, having a mass of $1.25 \mathrm{~kg}$, resulting in a thermal mass of $250 \mathrm{JK}^{-1}$ to $375 \mathrm{JK}^{-1}$ in the temperature range of $80 \mathrm{~K}$ to $140 \mathrm{~K}$. As a consequence, the reservoir is expected to increase in temperature at most by $1 \mathrm{~K}$ for every cooling procedure.

(vii) Gas traversing in the gas-gap experiences constant temperatures along both the walls of the parallel plates

The characteristics time for the cooling of the cryo-vial is in the order of few seconds, which is much larger compared to the gas residence time of less than $10 \mathrm{~ms}$ in the gas-gap for the mass flow rate of $40 \mathrm{mgs}^{-1}$. Therefore, a gas parcel traversing along the length of the parallel plate will not notice a change in the temperature of the walls of the parallel plate (note that one of the walls of the parallel plate represents cryo-vial whose temperature decreases during cooling, but this process is slow for a gas parcel to notice the change in temperature).

(viii) The gas-gap size is constant in the longitudinal direction of the vial.

\section{Model description}

For forced flow in the gas-gap, three effects are identified: Firstly, the length changes for which convection in the gap plays a role. The enhanced heat transfer across the gap is however a minor effect, since it is much smaller than the length of the gap. This convection-length can be estimated using classical duct-flow analysis and yields $\ell(U) \sim \frac{d^{2} U \alpha}{2 \cdot \mathscr{N} u}$. Here $\mathscr{N} u=7.54$ the Nusselt number for parallel plates, well suited since the gap $d \ll R$. For the flows studied we obtain a maximum $\ell \approx 1 \mathrm{~mm}$ for $\dot{m}=40 \mathrm{mg} \mathrm{s}^{-1}$, much smaller than the length of the vial, being $22 \mathrm{~mm}$. This implies that beyond $\ell$ convection is not important and no enhancement of heat transfer occurs, it is solely conduction from the vial to the TESU.

Secondly, the jet characteristics change significantly for higher flow rates. This causes thinning of the thermal boundary layer at the bottom of the vial, 
resulting in an enhanced cooling from this area (see Figure 5.4). Nevertheless, the area of the bottom is small compared to the sidewalls of the container, resulting in small increase in the cooling rate, which is quantified in the next chapter using a numerical model.

Thirdly, the gas undergoes a change in temperature when flowing past the vial. Initially, the gas is at the temperature of the TESU, as it is cooled by the cryocooler using a heat exchanger. When it flows past the hot vial, the gas heats up. To simplify the enthalpy calculation for the gas exiting the gap, the temperature profile across the gap at the exit is assumed linear (for exact temperature profiles in the gas-gap see Appendix). The enthalpy increase with respect to the injection state is $\Delta \dot{H}=2 \pi \int_{\frac{D_{v}}{2}}^{\frac{D_{v}}{2}+d} \rho_{\mathrm{g}} c_{\mathrm{g}} u\left(T-T_{\infty}\right) \mathrm{d} x$, where $D_{v}$ is the diameter of the vial and $T$ and $u$ the temperature and velocity profiles in the gap respectively. This integral can now be approximated in the aforementioned case of a linear temperature profile, using the mass flow $\dot{m}_{\mathrm{g}}$ : $\Delta \dot{H}_{\mathrm{est}} \approx \frac{1}{2} \dot{m}_{\mathrm{g}} \bar{c}_{\mathrm{g}}\left(T-T_{\infty}\right)$, where $\bar{c}_{\mathrm{g}}$ is the gas specific heat at constant pressure evaluated at the mean temperature in the gap. The estimated values of $\dot{H}_{\text {est }}$ are also verified using a numerical model, which is discussed in the next chapter. Note that the residence time of the gas in the gap is much smaller compared to the cooling rate of the vial, justifying the assumption that the gas leaves the gap at approximately half the temperature difference between the vial and the TESU. Moreover, the total heat capacity of the gas leaving the gap per unit time is also much smaller than the total heat capacity of the vial. However, this approximation will also be validated using a numerical model in the next chapter. We therefore can predict the temperature of the vial using our approximation of $\Delta \dot{H}$ :

$$
\underbrace{-m_{v} c_{v}(T) \frac{d T}{d t}}_{\text {Total heat transfer }}=\underbrace{\varepsilon \bar{\lambda}_{\mathrm{g}}(T) A \frac{T-T_{\infty}}{d}}_{\text {Diffusion heat transfer }}+\underbrace{\frac{1}{2} \dot{m}_{\mathrm{g}} \bar{c}_{\mathrm{g}}\left(T-T_{\infty}\right)}_{\text {Advective heat transfer }} .
$$

Re-writing this equation,

$$
-C(T) \frac{d T}{d t}=\frac{\left(T-T_{\infty}\right)}{R(T)}
$$


in this, $C(T)=m_{v} c_{v}(T), R(T)=\left[\frac{\varepsilon \bar{\lambda}_{\mathrm{g}}(T) A}{\delta_{\mathrm{g}}}+\frac{\dot{m}_{\mathrm{g}} c_{\mathrm{g}}}{2}\right]^{-1}$ and the constant $\varepsilon$ is the fitting parameter responsible for enhanced heat transfer due to misalignment of the vial in the gas-gap, which will be discussed in the next chapter. The equivalent thermal network of the vial thermal interaction is represented as,

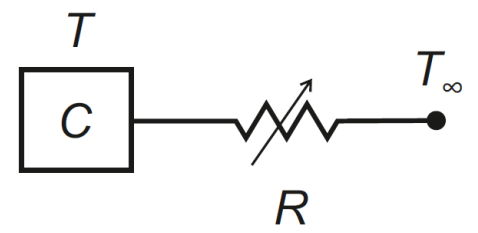

Figure (5.5) Equivalent thermal network

Using, Equation 5.7, temperature of the vial can be predicted during its cool-down in the gas-gap. This concludes the design of the tissue snap-freezer. In the next chapter, results obtained using the heat transfer model discussed above is validated using the measurements performed for different heat sink temperature $T_{\infty}$, mass flow rate of helium gas $\dot{m}_{g}$, and different gases.

\subsection{Conclusions}

A device to freeze bio-samples enclosed in a vial is developed, which is powered by a low capacity cryocooler. To accomplish this, different cooling principles are assessed to meet the snap-freezer requirements. Among all the principles, forced convective gas-flow meets all the requirements and therefore is chosen as the cooling principle for the device. A design methodology for the individual components of the snap-freezer is also discussed. A pulse tube cryocooler rated at $4 \mathrm{~W}$ at $80 \mathrm{~K}$ provides the cooling to a Thermal Energy Storage Unit (TESU) made up of $1.25 \mathrm{~kg}$ of copper mass. To reduce the size of the TESU, a counter flow heat exchanger is included to pre-cool the warm helium gas flow to the apparatus. We also developed a heat transfer model to predict temperature variation of a commercial aluminum vial cooled inside the snap-freezer. 


\section{Appendix - III}

\section{Gas properties:}

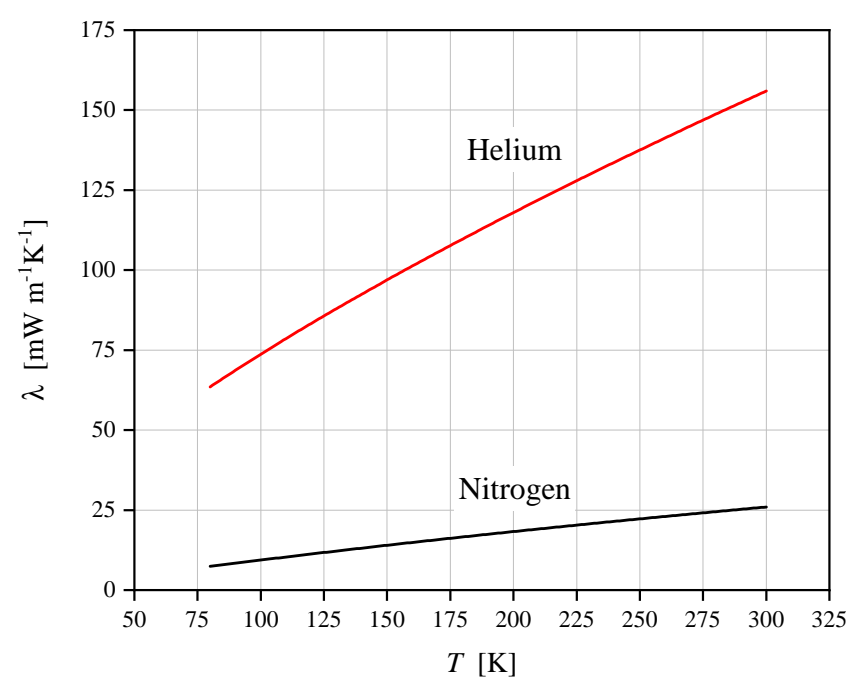

(a) Thermal conductivity.

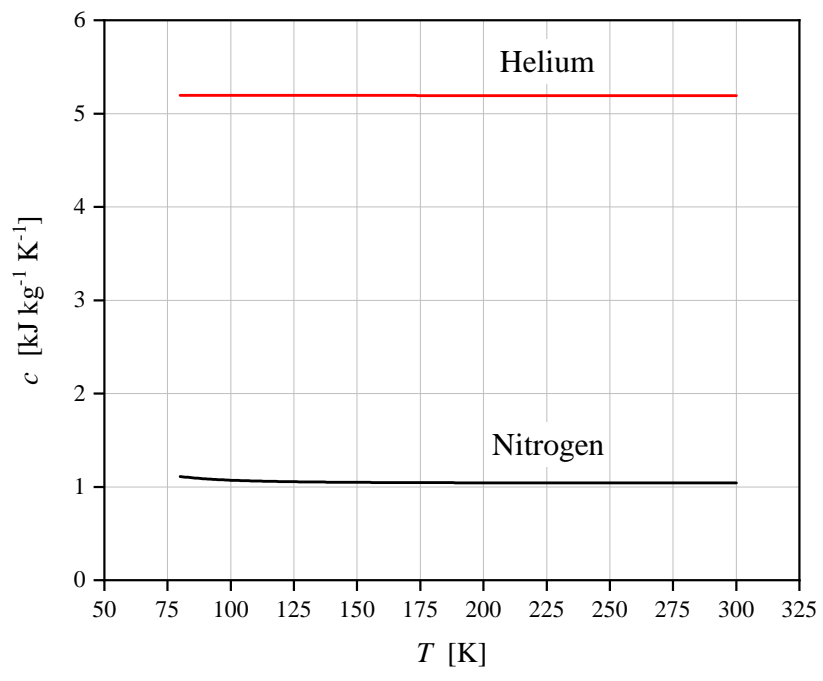

(b) Specific heat capacity. 


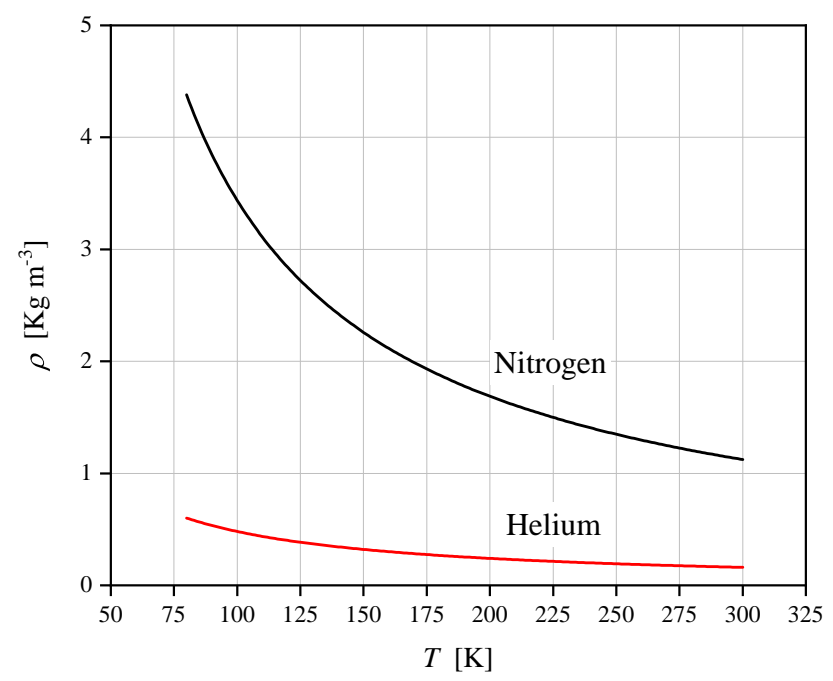

(c) Density at 1 bar.

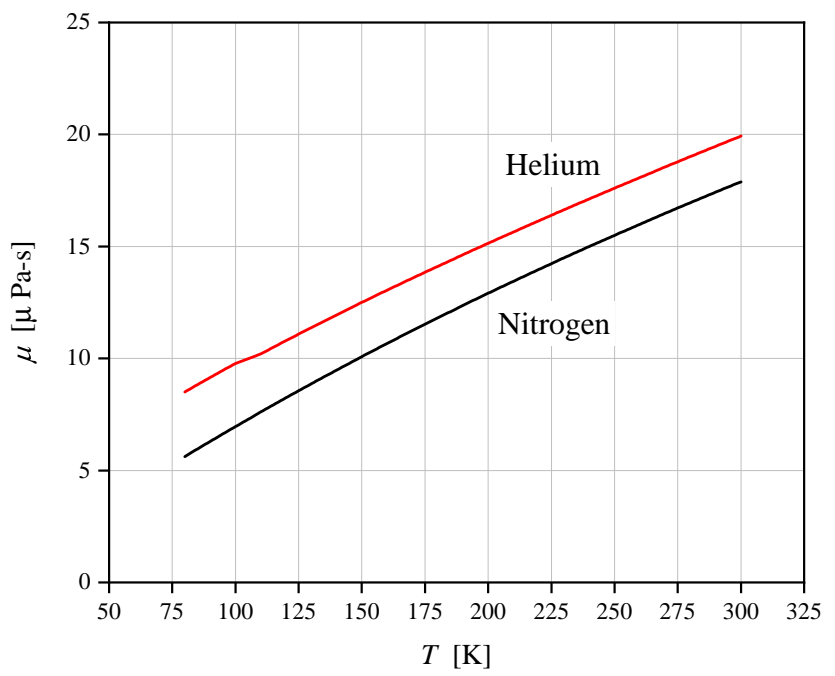

(d) Viscosity at 1 bar.

Figure (5.6) Properties of the used working gases. 


\section{Aluminum and copper thermal properties:}

The thermal conductivity and specific heat of both copper and aluminum are shown in Figure 5.7.

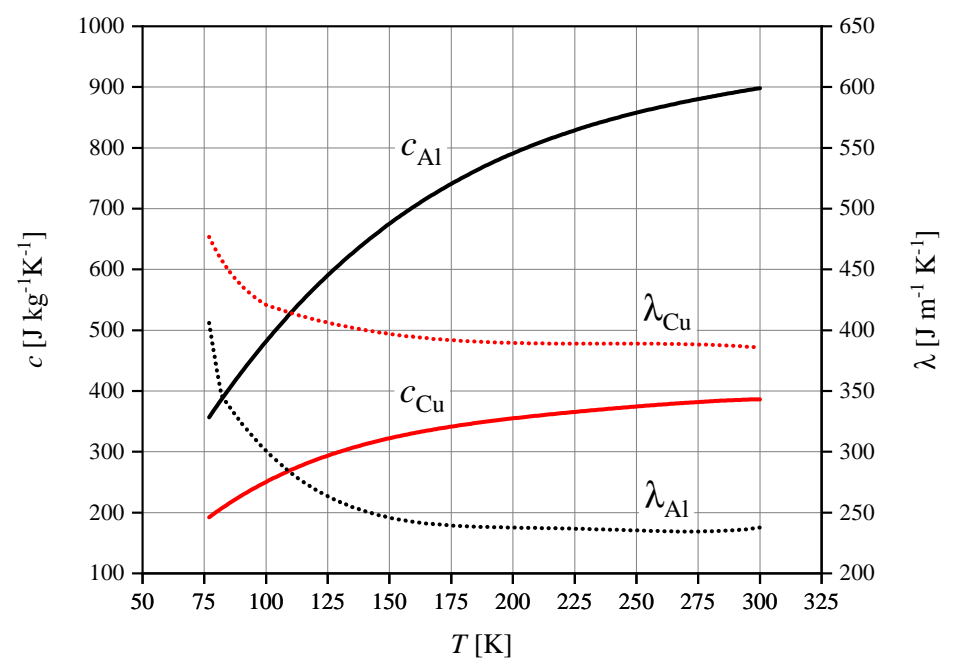

Figure (5.7) Specific heat capacity $(c)$ and thermal conductivity $(\lambda)$ of the Aluminum [29-31] and Copper [30, 31, 62] material.

\section{Temperature profile inside the gas-gap:}

The thermal properties of the gas vary linearly with temperature and therefore, the temperature profile inside the gas-gap will not be linear and can be estimated using the Fourier's heat equation,

$$
-\lambda_{\mathrm{g}} \frac{d T}{d x}=q^{\prime \prime}=\text { constant } .
$$

The thermal conductivity of the gases in the viscous regime varies linearly with temperature, i.e. $\lambda_{\mathrm{g}}=B_{1}+C_{1} T$, where $B_{1}$ and $C_{1}$ are the constants corresponds to a particular gas and are given in Table 5.4. Therefore, the steady-state temperature profile inside the gas-gap can now be calculated by integrating the Equation 5.8 with respect to $x$, using the boundary conditions, at $x=0, T=T_{\text {vial }}$ and $x=d, T=T_{\infty}$. The expression for the temperature 
profile in the gas-gap is now given by,

$$
\left(B_{1} T+C_{1} \frac{T^{2}}{2}\right)=\left[\frac{1}{L}\left(B_{1}\left(T_{\infty}-T_{\text {vial }}\right)+\frac{C_{1}}{2}\left(T_{\infty}-T_{\text {vial }}\right)\right] x+\left[B_{1} T_{\text {vial }}+C_{1} \frac{T_{\text {vial }}^{2}}{2}\right]\right.
$$

\begin{tabular}{|c|c|c|}
\hline Gas & B1 & C1 \\
\hline Helium & $33.49 \times 10^{-3}$ & $0.4161 \times 10^{-3}$ \\
\hline Heliox & $18.70 \times 10^{-3}$ & $0.3283 \times 10^{-3}$ \\
\hline Nitrogen & $1.2396 \times 10^{-3}$ & $0.0841 \times 10^{-3}$ \\
\hline
\end{tabular}

Table (5.4) Thermal conductivity constants for gases

Figure 5.8 shows the calculated temperature profiles in the gas-gap assuming a fixed temperature equal to $80 \mathrm{~K}$ on one side and for various temperatures on the other side of the gap. A gas-gap size of $0.4 \mathrm{~mm}$ corresponding to the gas-gap size in the snap-freezer is used here.

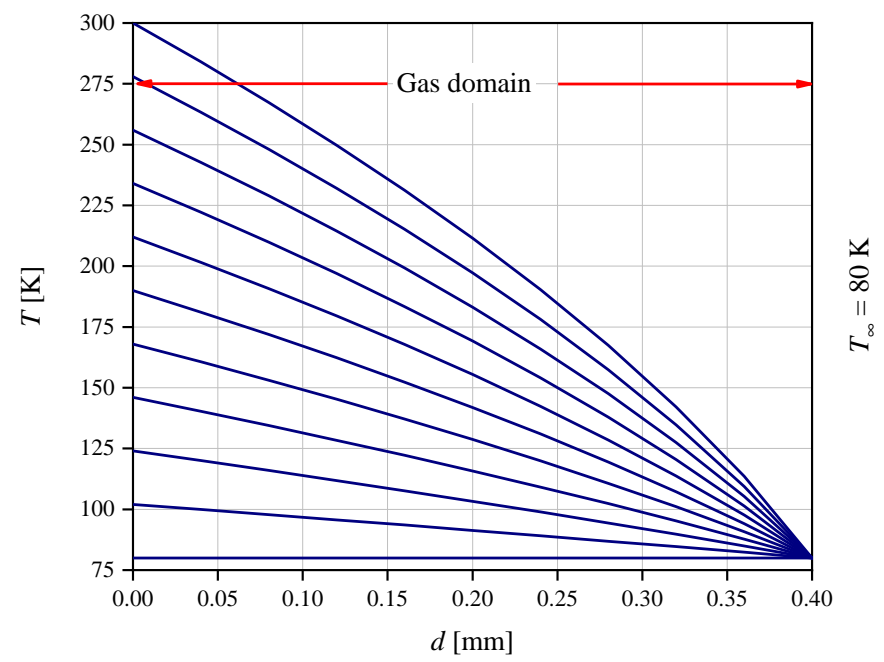

Figure (5.8) The calculated helium gas temperature in a gas-gap formed by two parallel plates of gap $0.4 \mathrm{~mm}$ with one plate fixed at $80 \mathrm{~K}$ and the temperature of the second plate is varied. 


\title{
Tissue snap-freezer: measurements and model validation $^{1}$
}

\begin{abstract}
In this chapter, the hardware and assembly of the snap-freezer built is discussed. The heat transfer model developed to predict the cooling of the vial is validated using the measurements performed with the snap-freezer. We systematically study heat transfer during cooling of the vial for several settings of the snap-freezing device namely heat sink temperature $\left(T_{\infty}\right)$, gas mass flow rate $\left(\dot{m}_{\mathrm{g}}\right)$ and gas thermal conductivity $\left(\lambda_{\mathrm{g}}\right)$. Cooling experiments performed with the snap-freezer show that cooling speeds higher than with quenching in liquid nitrogen is achievable. Using the heat transfer model it is also shown that the narrow gas gaps increases the heat transfer rate substantially, com pared to the marginal increased cooling rate due to forced flow of the gas.
\end{abstract}

\subsection{Materials and methods}

Figure 6.1 shows the CAD model and the hardware components of the snapfreezer. The functional description and the design strategy of all the components including cryocooler, TESU, counter flow heat exchanger (CHFX) and cold heat exchanger (CHX) is discussed in the Chapter 5. In this section, the assembly of these components is discussed. For CHFX, a tube in tube configuration is used in which the tube length is $4.8 \mathrm{~m}$ and the diameter for the inner and outer tube is $2.4 \mathrm{~mm}$ and $5.2 \mathrm{~mm}$ respectively. The cold gas exiting from the TESU enters in to the CHFX, which then after exchanging the cold with the incoming gas at room temperature exits into the atmosphere. The

\footnotetext{
${ }^{1}$ Published as: van Limbeek, M.A., Jagga, S., Holland, H., Ledeboer, K., ter Brake, M. and Vanapalli, S., 2019. Cooling of a vial in a snapfreezing device without using sacrificial cryogens. Scientific reports, 9(1), pp.1-9.
} 
incoming gas after exchanging cold enters into the CHX, which is in direct contact with the TESU and therefore cools the gas to the set temperature of the TESU. A tube of $0.47 \mathrm{~m}$ in length and $3.1 \mathrm{~mm}$ in diameter is used for the $\mathrm{CHX}$. The cold gas exiting from the CHX enters into the hole made to insert the vial from the botton of the TESU.

The TESU is directly placed in contact with the cryocooler to control its temperature. A pulse tube cryocooler of the type LPT9310 manufactured by Thales Cryogenics is used in our experiments. The compressor and the warm end of the pulse tube are forced air cooled by a fan. The electronics supplied with the cryocooler allows setting the desired cold temperature. The temperature of the cold heat exchanger is measured using a silicon diode sensor, which has a maximum error of $\pm 0.5 \mathrm{~K}$ for the temperature range of $54 \mathrm{~K}$ to $300 \mathrm{~K}$. The hole to accommodate the vial in the TESU is maintained at a distance from the atmosphere using a bellow tube made up of stainless steel; this is done to reduce the heat leak and moisture deposition on the TESU.

To start the cooling experiments, the snap-freezer is prepared by cooling the Thermal Energy Storage Unit (TESU) with a cryo-cooler to a set cold temperature and circulating a low flow of gas, about $4 \mathrm{mg} . \mathrm{s}^{-1}$. The flow of gas cools the counter flow heat exchanger and brings the system to a steady cold state. During insertion of the cryo-vial, a low flow rate of gas is maintained to prevent moisture deposition in the apparatus. Once the vial is inserted, the flow is increased to the set value depending on the desired cooling rate. The working gas is fed from a gas bottle and the flow is controlled by a flow controller. The gas flows to the snap-freezer and is vented into the lab afterwards. After the specified cool down time, the circulating gas flow rate is reduced and the cryo-vial is removed from the apparatus. Some experiments are also performed with the static gas in the gas-gap i.e., no gas flow, in which during the insertion and removal of the cryo-vial some amount of the static gas escapes to the ambient which is then replenished by the gas-handling system to prepare the setup for the next experiment.

To perform the cool-down measurements, three type-E thermocouples are placed along the inner wall of the vial to measure its temperature. Each thermocouple is attached to the wall using a tiny piece of aluminum tape. All three thermocouples reads out the same temperature variation, which ensures its good thermal contact with the inner wall. To demonstrate the repeatability, 
multiple experimental runs were conducted for each measurement. The thermocouple wire is inserted into the cryo-vial through a small hole drilled on the cap, the hole is then closed using a small amount of the stycast 2850FT material. The amount of stycast and aluminum tape used is very less and have heat capacity values very low compared to the aluminum vial and therefore does not effect its cool-down.
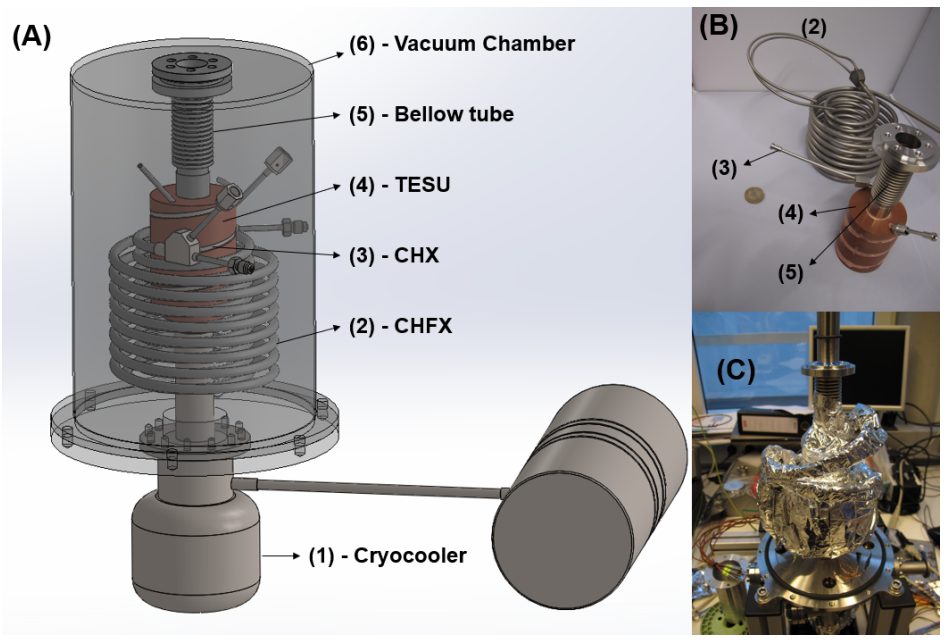

Figure (6.1) (A) - CAD model of the snap-freezer showing all its components. (B) - Actual pictures of the components. (C) - The components numbered 2 to 5 wrapped using multi-layer insulation (MLI).

\subsection{Results and Discussion}

\subsubsection{Cooling affect by thermal diffusion}

We first perform experiments with the TESU cooled to $T_{\infty}=80 \mathrm{~K}$. To obtain a qualitative comparison to the reference case of quenching the vial in liquid nitrogen, a separate experiment carried out in our lab. Here we submerged the vial, initially at $T=T_{\mathrm{amb}}$ into a bath of liquid nitrogen and recorded the temperature during cooling. The averaged results for four cooling measurements in our snapfreezer are presented in Figure 6.2. Aside from the delay of half a second as a result from the vial insertion protocol, we find excellent reproducibility: The largest difference between the four measurements was only $3 \mathrm{~K}$. We now compare the data with the solution of Equation 5.7. Both the model and the experimental data cool down much faster for $T>120 \mathrm{~K}$ than 
quenching in liquid nitrogen. Though the model already describes the cooling behaviour quite well, better agreement can be achieved when allowing for an amplification of heat flux by a factor $\varepsilon$, as can be seen by the yellow curve. With only a slight increase of $11 \%$ we obtain much better agreement.

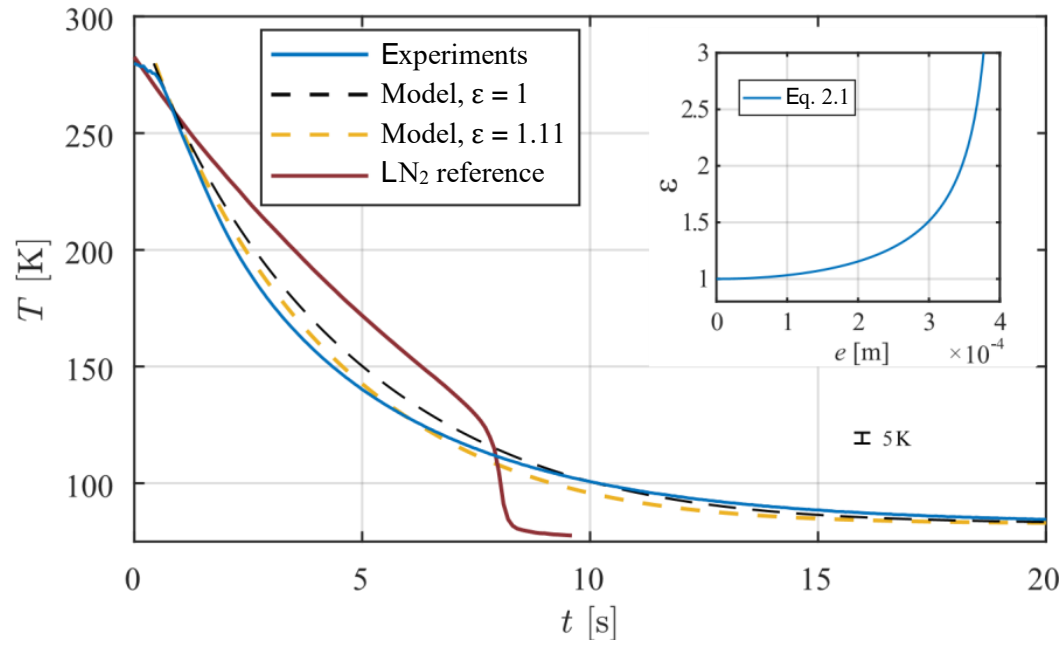

Figure (6.2) The experimental data is the average of four different measurements, which differed at most $3 \mathrm{~K}$, smaller than the line thickness. The inset shows the enhancement in heat transfer $\varepsilon$ as a result of a improperly centered vial, described by Equation 6.1

We now explore several possible origins of this enhancement. Firstly, when the vial axis is not concentric to the device axis, an enhancement in heat transfer $S(e)$ occurs, depending on the eccentricity $e$, which is the displacement of the vial axis from the device centre. For $e=0$, the vial is placed exactly in the centre, while for $e=0.4 \mathrm{~mm}$ the vial is touching the wall of the TESU. The enhancement in heat transfer is given by [45],

$$
\varepsilon=\frac{S(e)}{S(0)}=\frac{\cosh ^{-1}\left(\frac{D_{v}^{2}+\left(D_{v}+2 d\right)^{2}}{2 D_{v}\left(D_{v}+2 d\right)}\right)}{\cosh ^{-1}\left(\frac{D_{v}^{2}+\left(D_{v}+2 d\right)^{2}-e^{2}}{2 D_{v}\left(D_{v}+2 d\right)}\right)}
$$


in this, $D_{v}$ is the vial outer diameter. In the inset of Figure 6.2, $\varepsilon$ is shown for various values of $e$. We find the best fit to the data using $\varepsilon=1.11$, corresponding with an eccentricity of $0.17 \mathrm{~mm}$. This however is not predictable, since the vial is inserted manually. The effect of eccentric placement of vial is also studied using numerical methods, which is discussed later in the section 6.3.

Secondly, any tilting of the vial inside the TESU makes one side at the top and the opposite side at the bottom of the vial approach closer to the wall. From the geometry we calculated that the maximum tilt was $1.9^{\circ}$, which results in an enhanced heat transfer of $\varepsilon=1.48$ as found by a numerical study of this effect (see the section 6.3).

Thirdly, we find a faster cooling rate of the vial in our measurements for the first two seconds (see Figure 6.2). This might be a result of the insertion method, where the gas in the TESU is displaced by the vial. As a result of this, the gas flows out of the TESU, enhancing the cooling rate. This effect however decays over time as a result of the viscosity. In the next section, the measurements performed for different heat sink temperature are compared with the heat transfer model.

\subsubsection{Vial cool-down with different heat sink temperature}

As the system allows to study the effect of freezing conditions on the quality of the biological material stored in the vial. Therefore, we test the apparatus for different TESU temperatures $T_{\infty}$. For every measurement, the vial is taken out of the snap freezer, allowing the vial to equilibrate to the temparature of the lab. Since each experiment involves a new insertion procedure, the positioning is expected to differ for every measurement, leading to a different $\varepsilon$. Results of four averaged cooling measurements are presented with the solution of Equation 5.7 in the Figure 6.3 for three different set point temperatures $T_{\infty}$. It is clearly visible that the cooling rate can be varied by the set point temperature, since $\frac{d T}{d t} \propto T-T_{\infty}$. For $T_{\infty}<140 \mathrm{~K}$, we find that the cooling rate is higher in the liquid nitrogen in the temperature range of $300 \mathrm{~K}$ to $220 \mathrm{~K}$, so called danger zone where ice crystals may damage the tissue [76]. The cooling rate is well captured by our one-dimensional model. However a higher cooling rate is observed initially, most likely as a consequence of forced convection originating from the insertion method, as described in the previous 
section above. This effect has decayed after a few seconds and the predicted cooling rate is within $15 \%$ of the experimental value.

The non-exponential cooling characteristic in liquid nitrogen lays in the fact that during cooling the film thickness becomes smaller, while in our apparatus we have a constant gas-gap thickness. The main contribution to the rapid cooling however is that in our experiments we used helium gas, which has a high thermal conductivity compared to nitrogen. It is therefore interesting to compare the performance using different gases in the gap, which are discussed in the next section.

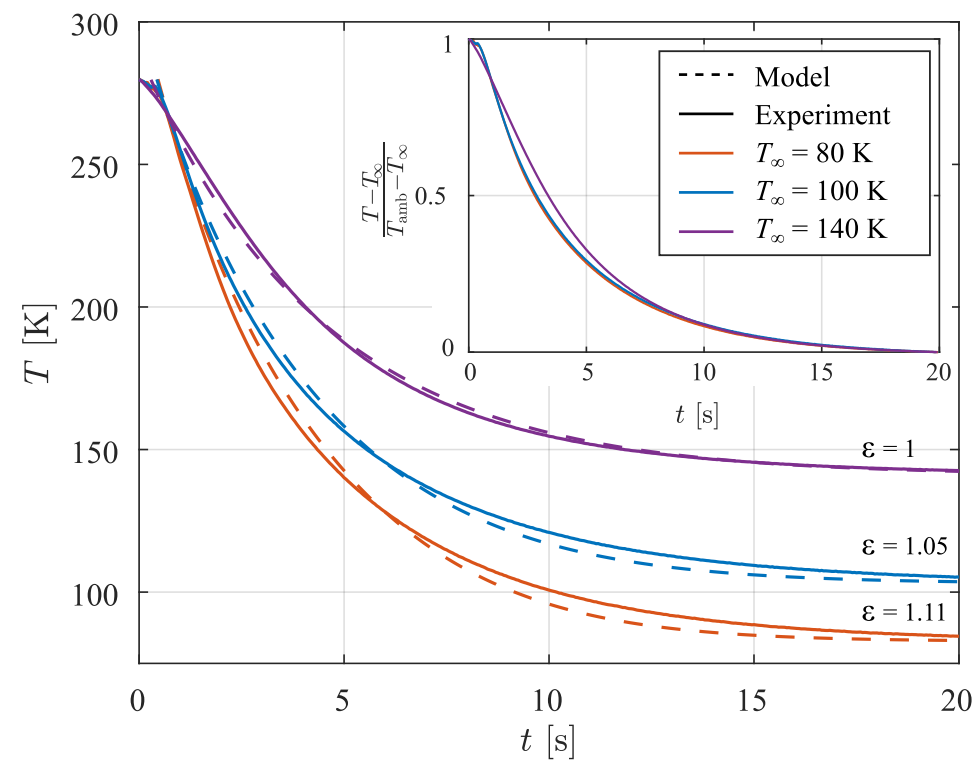

Figure (6.3) Comparison of experimental data for different heat sink temperatures.

\subsubsection{Vial cool-down with various gases in the snap-freezer}

To see the effect of gas thermal conductivity on the cool-down, three types of gases are used, namely helium, nitrogen, and heliox. The latter is a mixture of both helium and oxygen in a composition of 79 to 21 mass percent, respectively. Heliox is frequently used in hospitals, which makes it easy to use for the snap freezer. Prior to insertion we flush the insertion volume with the gas, then stop the flow and insert the vial into the snap-freezer. We measure 
the temperature $T$ during cool down, which are displayed in Figure 6.4. As expected, the vial in case of nitrogen in the gas-gap cools down much slower compared to the helium and heliox gas as a result of the lower thermal conductivity. A small difference can be found for Heliox, compared to helium, since it contains $21 \%$ of oxygen, lowering the conductivity of the gas mixture.

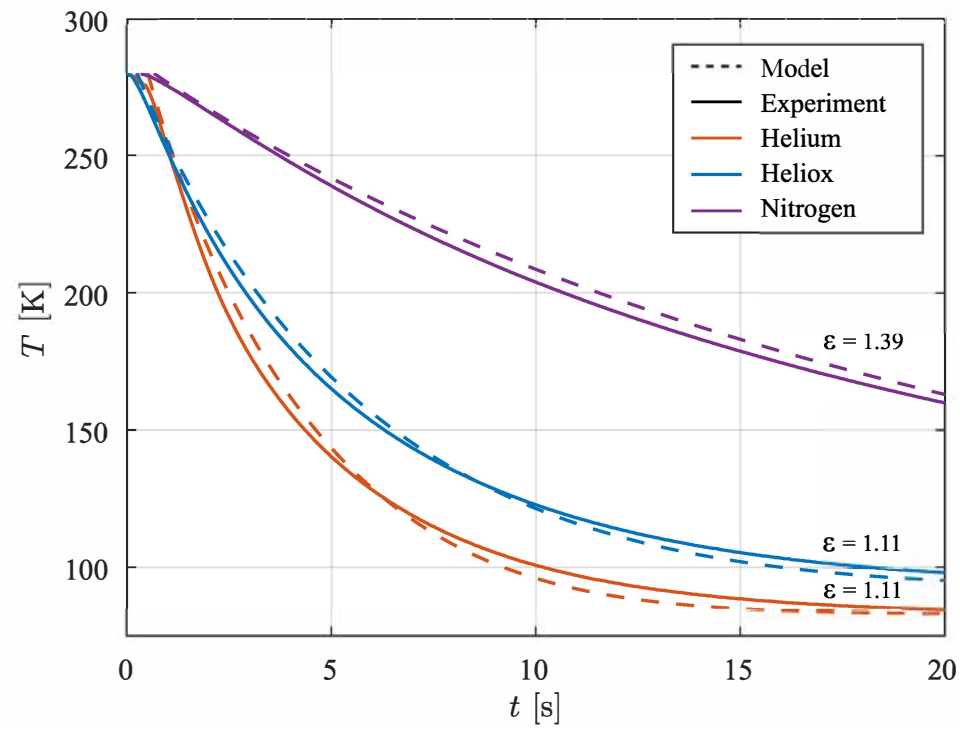

Figure (6.4) Comparison of experimental data of cryo-vial quenching with helium, nitrogen and heliox in the gas gap with the model

\subsubsection{Vial cool-down with different mass flow rate in the snap- freezer}

As mentioned before, gas can be injected to flush the system. Where before the flow was stopped before the insertion of the vial, we here study cooling of the vial where the gas is still flowing. The gas is injected from below, impacting the bottom of the vial and then flowing through the gap between the TESU and the side wall of the vial. The results for a flow rate of 20 and $40 \mathrm{mg} \mathrm{s}^{-1}$ of helium is shown in the Figure 6.5. It can be seen that the cooling rate is enhanced with increasing flow rate. However, the flow has only a small influence on the cooling of the vial. 


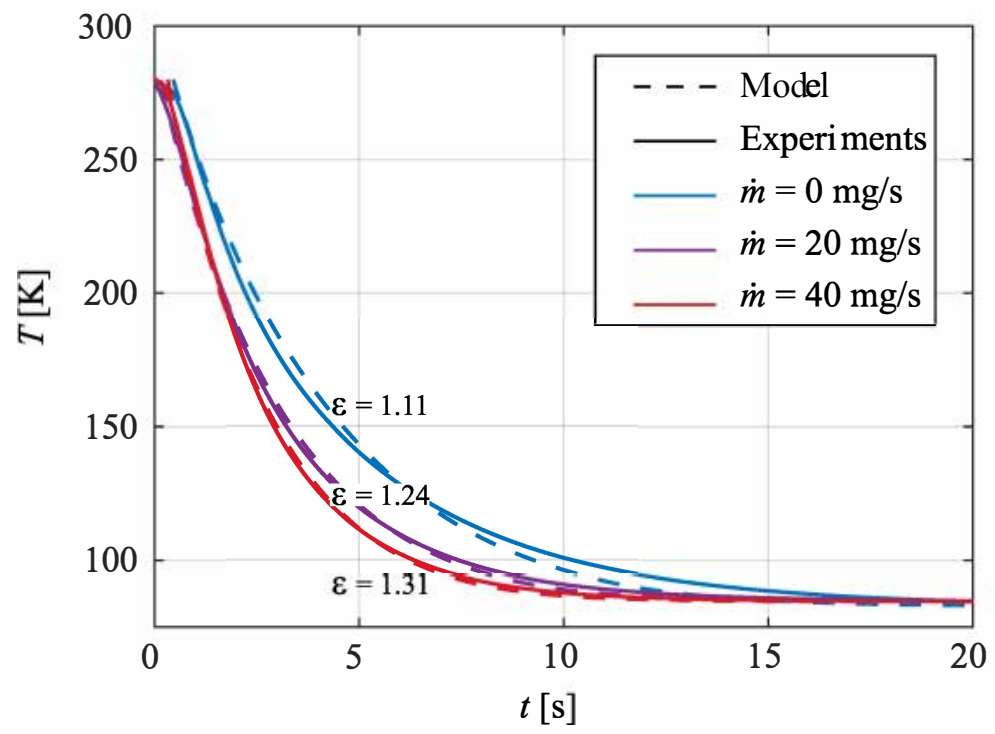

Figure (6.5) Cooling results for the vial in the presence of a continues gas flow jet at various flow rates of helium.

By the aid of a commercially available finite elements simulation software [77] we simulated the effect of the flow on the cooling rate (details can be found in the section 6.3). Since we consider a quasi-static problem, the divergence of the enthalpy field should be zero. Applying the divergence theorem one can thus evaluate the fluxes at the boundary to investigate how the flow alters the energy fluxes in the system. Simulations were performed with a helium flow up to $40 \mathrm{mg} \mathrm{s}^{-1}$, comparable with experimental conditions. The results are listed in the Table 6.1, where the various heat fluxes are calculated, together with the change in enthalpy of the gas $\Delta H$, which will be discussed later. We find that the majority of the heat exchange still occurs by diffusion across the thin gap. Only flow rates larger than $20 \mathrm{mg} \mathrm{s}^{-1}$ increased the heat flux by more than $10 \%$, see Table 6.1 .

The increased cooling rate caused due to thinning of the thermal boundary layer at the bottom of the vial is calculated approximately $10 \%$ for the highest flow rate of $40 \mathrm{mg} \mathrm{s}^{-1}$. The estimated values of the increased enthalpy, $\Delta \dot{H}_{\text {est }} \approx \frac{1}{2} \dot{m} \bar{c}_{\mathrm{g}}\left(T-T_{\infty}\right)$, are also compared with the calculated values using the numerical model in the Table 6.1. It can be seen that this approximation deviates the values only by $10 \%$. 


\begin{tabular}{c|cccccc}
$\begin{array}{c}\text { Mass flow } \dot{m} \\
{\left[\mathrm{mg} \mathrm{s}^{-1}\right]}\end{array}$ & $\begin{array}{c}\dot{Q}_{\text {vial }} \\
{[\mathrm{W}]}\end{array}$ & $\begin{array}{c}\dot{Q}_{\text {TESU }} \\
{[\mathrm{W}]}\end{array}$ & $\begin{array}{c}\dot{Q}_{\text {outlet }} \\
{[\mathrm{W}]}\end{array}$ & $\begin{array}{c}\Delta \dot{H}_{\text {est }} \\
{[\mathrm{W}]}\end{array}$ & $\begin{array}{c}\dot{Q}_{\text {vial }}(\dot{m}) \\
\dot{Q}_{\text {vial }}(\dot{m}=0)\end{array}$ & $\frac{\dot{Q}_{\text {outlet }}}{\dot{Q}_{\text {vial }}}$ \\
\hline 0 & -68.2 & 68.0 & 0 & 0 & - & - \\
4 & -71.1 & 69.1 & 1.8 & 2.0 & 1.04 & 0.03 \\
20 & -80.0 & 70.1 & 9.0 & 10.4 & 1.17 & 0.11 \\
40 & -88.7 & 69.0 & 18.0 & 20.8 & 1.30 & 0.20
\end{tabular}

Table (6.1) Simulation results for a vial at $T=290 \mathrm{~K}$ and the TESU at $80 \mathrm{~K}$, where a helium gas flow at $80 \mathrm{~K}$ is forced around the vial. $\dot{Q}_{\text {vial }}, \dot{Q}_{\mathrm{TESU}}$, and $\dot{Q}_{\text {outlet }}$ are the heat flows from the vial, TESU and carreid by the flow, respectively. As a reference, evaluation of Equation 5.7 for the same temperature difference yields $65.3 \mathrm{~W}$.

Based on our measurements and the simulations, we can conclude that the effect of convection by continuous gas flow is small as a result of the high ratio between the length of the vial and the small gap. Most energy is still transferred in the form of diffusion across the gap, while the heating of the cold gas accounts for at most $20 \%$ of increased cooling rate for the mass flow rate of $40 \mathrm{mgs}^{-1}$. An indirect enhancement in this diffusion is also noted. We observe in Figure 6.5 that $\varepsilon$ also increases with increasing flow rate. For a purely viscous flow the centre configuration is unstable and any misalignment forced the vial more out of centre. Overall, the usage of gas injection is not the most optimal way to vary the cooling rate of the vial, as large quantities of gas are discharged and lost to the environment. The cooling rate can be varied more easily by adjusting the temperature $T_{\infty}$ of the TESU.

The mathematical analysis presented in this paper and its validation with experimental data will allow exploration of the parameter space of the snap freezing device so as to design a cost effective apparatus; as an example nitrogen gas with a narrower gas-gap of $0.1 \mathrm{~mm}$ will have similar cooing rate as liquid nitrogen. Adapters to accommodate various gas-gap sizes could also be built, which is discussed in the next section.

\subsection{Numerical methods}

We used a commercial finite elements package [77] to simulate two aspects of vial positioning in the TESU, as well as the influence of convection. For all these studies, we used the geometry and materials as described in the pre- 
vious chapter. The justification of the lumped-capacity model was demonstrated there as well, and hence the simulations were performed by seeking the steady-state solution of the system, where we modeled only the gas domain. The quasi-static cooling of the vial was simulated by varying the boundary condition of the gas domain, while keeping the opposite boundary condition fixed at the TESU temperature $T_{\infty}=80 \mathrm{~K}$. The advantage of solving the steady state problem is that it is not computationally expensive.

In the first two numerical studies we neglected diffusion from the top and bottom of the vial, as the analytical model do not include these as well. The vial is thus modeled as a cylinder, where the vial and the lid are modeled as one piece. From the large difference between the vial radius and the maximum eccentricity, which is the gap size, this effect is indeed negligible. The limit of direct contact was not explored as the diverging heat flux will induce local cooling effects, which is beyond the present scope of the study. As explained in the paper, one can capture any temperature dependency of the thermal conductivity by using an effective thermal conductivity. For simplicity, we used a constant conductivity here, so we only solve $\nabla^{2} T=0$. We then evaluate the fluxes on the vial wall $\int_{A} \vec{q} \cdot \vec{n} \mathrm{~d} A$, where $A$ and $\vec{n}$ are the area and normal of the vial side-wall respectively and $\vec{q}=\bar{\lambda}_{\mathrm{g}} \nabla T$ is the local heat flux. Since we are interested in the enhancement in heat transfer and the problem is linear, the exact values for the temperature differences and conductivities are not important here as they cancel after the normalization. A sketch of the shifted or tilted vial can be seen in Figure 6.6.

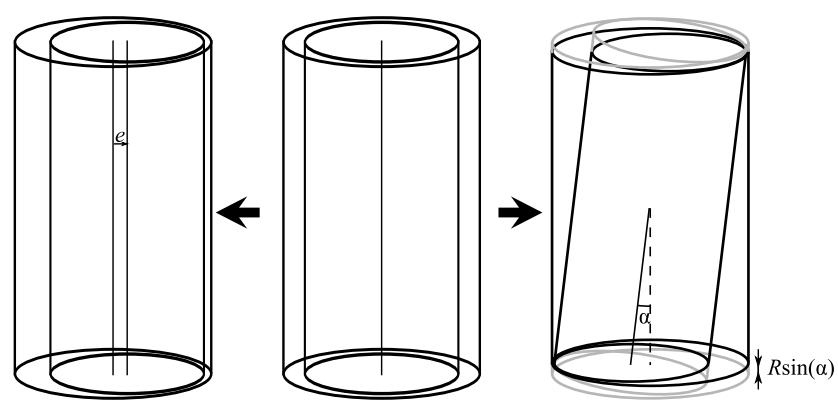

Figure (6.6) Sketch of a vial being shifted (left) from the center or tilted (right) 


\section{Effect of Eccentricity}

Here, we validated the enhancement in heat transfer $\varepsilon$ by an eccentric positioning and found good agreement between the analytical (Equation 6.1) and the numerical solution, see Table 6.2 .

\begin{tabular}{l|lllll} 
Eccentricity $e[\mathrm{~m}]$ & 0 & $1 \times 10^{-4}$ & $2 \times 10^{-4}$ & $3 \times 10^{-4}$ & $3.9 \times 10^{-4}$ \\
\hline$Q(e) / Q(0)$ & 1 & 1.03 & 1.15 & 1.51 & 4.31 \\
Equation 6.1 & 1 & 1.03 & 1.16 & 1.51 & 4.45
\end{tabular}

Table (6.2) Numerical validation of Equation 6.1, where an enhancement in heat flux occurs as a result of an eccentric position $e$ of the vial in the snapfreezer. Though one side of the vial is moving away from the side wall with increasing $e$ the opposite side gets closer, leading to an increase in total heat flux.

\section{Affect of tilt inside the gas-gap}

Now, we positioned the vial again in the center $(e=0)$ and tilted it inside the gap. The maximum tilt achieved was $1.9^{\circ}$, in agreement with geometrical calculations. In this position, the top of the vial is touching the wall of the TESU, while on the same side, the bottom is almost at $0.8 \mathrm{~mm}$ away from the wall. On the opposite side of the vial, the top is away from the TESU, while the bottom makes contact. The increase in total heat flux is presented in Table 6.3, where we see that the maximum increase in heat transfer $\varepsilon$ is 1.48 times the value for the case of zero tilt.

\begin{tabular}{l|llllll} 
Tilt angle $\left[{ }^{\circ}\right]$ & 0 & 0.5 & 1 & 1.5 & 1.75 & 1.9 \\
\hline$Q / Q\left(0^{\circ}\right)$ & 1 & 1.02 & 1.06 & 1.16 & 1.27 & 1.48
\end{tabular}

Table (6.3) Calculated enhancement in heat flux as a result of an tilted position of the vial in the snapfreezer.

\section{Effect of gas flow in the gas-gap}

Let us now consider the case that a helium flow is forced around the vial. The gas flow is injected from the bottom via the tube. The simulation is still steady state, as explained before and we assume an axi-symmetric domain, as the vial is positioned in the axis of symmetry (i.e. no tilt and eccentricity). We solve the flow field, allowing for compressibility effects as a result of the large 
density differences expected from the large temperature difference between the vial and the TESU wall (which can be direct after insertion $193 \mathrm{~K}$ ). We thus solve:

$$
\nabla \cdot(\rho \vec{u})=0 ; \quad \rho(\vec{u} \cdot \nabla(\rho \vec{u}))=-\nabla p+\nabla \cdot\left(\mu\left(\nabla \vec{u}+(\nabla \vec{u})^{T}\right)-\frac{2}{3} \mu(\nabla \cdot \vec{u}) \mathbf{I}\right)
$$

where $\rho$ and $\mu$ are the gas density and viscosity respectively and $p$ is the pressure field. I is the identity matrix and ${ }^{T}$ the transpose operator Equation 6.2 is coupled to the heat equation:

$$
\nabla \cdot(\lambda \nabla T)=0,
$$

in this, $\lambda$ is the thermal conductivity of the gas. All gas properties are evaluated locally, since they depend on the local temperature. The problem is closed using the equation of state, coupling the pressure field, density and temperature, where the ideal gas law was used:

$$
\rho=p M /(R T),
$$

where $M$ is the molar mass of the gas and $R=8.314 \mathrm{~J} \mathrm{~K}^{-1} \mathrm{~mol}^{-1}$ the universal gas constant. The problem is subject to an open boundary at the top $\left(\nabla p=0, p=10^{5} \mathrm{~Pa}\right)$ and no slip is applied on all solid boundaries. The vial wall is at $293 \mathrm{~K}$, while the tube and TESU are at $80 \mathrm{~K}$. We assume the injected gas flow to be fully developed and cooled to $80 \mathrm{~K}$. The mass flow is used as a control parameter.

We used a random triangulation of the domain, while rectangular boundary layers were used near the side walls. Additional refinement was imposed at the corners, capturing potential boundary layer separation. Over twelve thousand elements were used, and the solution was tested against coarser meshes to study the convergences of the solution.

The solution was found for various mass flow rates $\dot{m}$, for which the solution is still a laminar flow. The highest flow rate was found to be in the tube of the gas inlet. The heat transfer was evaluated at different positions: 1) the wall 
of the vial, using $\int_{A} \lambda \nabla T \cdot \vec{n} \mathrm{~d} A$, where $A$ is now the vial wall, bottom and top. 2) the change in enthalpy of the gas leaving the gap at the top of the vial: $\Delta H=2 \pi \int_{R}^{R+d} \rho_{\mathrm{g}} c_{p, \mathrm{~g}}\left(T-T_{\infty}\right) \vec{u} \cdot \vec{n} \mathrm{~d} x$ and 3) heat transferred from the gas into the TESU, evaluated similarly as 1). Evaluation of the heat exchanged at the top of the vial showed no significant contribution.

\subsection{Conclusions}

We performed measurements with the developed snap-freezer by varying different parameters such as cold temperature, gas flow rate and thermal properties of the gases. The contribution of heat transfer in the gas-gap due to the convection is small compared to the thermal diffusion through the gas-gap therefore, the gas flow through the gas-gap marginally influences the cooling speed of the vial. We verified the developed heat transfer model using the measurements. Using the heat transfer model we have also shown that the mis-alignment of the vial to the device axis results in the increased cooling speed. 
CHAPTER 7

\title{
Systematic approach to determine the transient cooling power and heat leak of a commercial pulse tube cryocooler. ${ }^{1}$
}

\begin{abstract}
In a typical pulse tube cryocooler application, modeling the cool-down dynamics of a load attached to the cold tip requires knowledge of the transient cooling power. Although this data may be calculated for in-house developed cryocoolers, the only data provided in commercial off-the-shelf cryocoolers is the steady state cooling power. In this paper, we show a systematic approach to derive transient cooling power data by performing heating and cooling experiments. The data obtained is verified with cooling data of an attached mass, which showed a good agreement. We also present a method to determine the parasitic heat load in a steady and transient operation of a commercial pulse tube cryocooler.
\end{abstract}

\subsection{Introduction}

The usage of cryocoolers once a workhorse for detector technology is branching into new application areas. To name a few, a device cooled by a pulse tube cryocooler to snap freeze human tissues for cancer diagnosis is developed by our team [78]. Recently, conduction cooling of superconducting radio frequency cavities for particle accelerators using a cryocooler was successfully

\footnotetext{
${ }^{1}$ Published as : Jagga, S., Holland, H.J. and Vanapalli, S., 2021. Systematic approach to determine the transient cooling power and heat leak of a commercial pulse tube cryocooler. Cryogenics, 113, p.103228.
} 
demonstrated $[79,80]$. Owing to low vibrations, there is an increasing interest in using a pulse tube cryocooler in high definition microscopy [81]. In addition to these new applications areas, commercial-off-the-shelf cryocoolers are also assessed in the detector community [82].

The data often provided by a cryocooler manufacturer are the load curve and the no-load cool-down of the cryocooler. The load curve is the net available steady-state cooling power as a function of cold-tip temperature. The cool-down characteristic is a plot of temperature measured at the cold heat exchanger as a function of time. The details such as the cold heat exchanger mass, transient cooling power, other internal geometry, and material parameters are often not provided as it is proprietary information of the manufacturer. However, in order to calculate the cooling dynamics in an application where a load is attached to the cryocooler, the data provided by the manufacturer is insufficient. For example, in a typical application using the steady-state cooling power values to determine the cool-down time of a mass attached to the cold-end will lead to a significant error.

A study by Grossmann et al. [83] reports a significant difference in the net steady-state and net transient cooling power values for a cryocooler. The reason for this difference is not clearly mentioned but is suspected to be due to different regenerator temperature profiles that lead to different parasitic heat loads at the cold end. This is an interesting outcome, as to date, transient effects inside the regenerator are not considered for the parasitic heat load calculations. For the steady-state operation, parasitic heat load values are usually approximated from transient measurements using a methodology reported by Vanapalli [84]. In this approach, first, the cooler is cooled to its minimum noload value, followed by turning off the compressor and allowing it to warm up while applying different amounts of heat input at the cold-end. The warm-up rate of the cold heat exchanger is then calculated using the temperature-time measurements. For any particular cold-end temperature value, the parasitic heat load is obtained by extrapolating the warm-up rate to zero.

Earlier attempts to determine the intrinsic cool-down time of a cryocooler were to attach several cold masses on the cold heat exchanger and measure the cool-down time. From these temporal temperature measurements and the procedure explained by Grossman et al. [83] and Vanapalli [85], the intrinsic cool-down time is derived. This method also allows the calculation of cooling 
transients with any load attached to the cold heat exchanger. However, it is to be noted that in the previous reported work, the inner dimensions of the cryocooler and the details of the components such as the heat capacity of the cold heat exchanger, porosity of the regenerator, effective cross-sectional area of the cold finger are known.

The objective of this paper is to show an experimental approach to determine the transient cooling power and parasitic heat leaks of a commercial offthe-shelf pulse tube cryocooler. First, we perform a pulse heating experiment to determine the effective cold mass of the cold finger, followed by cool-down with several continuous heating powers at the cold heat exchanger. The parasitic heat leaks are determined from heating experiments after the cryocooler has attained its lowest temperature. The tissue snap freezer developed by us consisted of a $1.25 \mathrm{~kg}$ copper mass attached to the cold heat exchanger of a $3.8 \mathrm{~W}$ at $80 \mathrm{~K}$ pulse tube cryocooler. The cooling of this mass is compared with the model that uses the transient cooling power determined in the above diagnostic experiments.

\subsection{Materials and procedure}

A pulse tube cryocooler of the type LPT9310 manufactured by Thales Cryogenics is used in our experiments. The compressor and the warm end of the pulse tube are forced air cooled by a set of two fans, one for the compressor and another for the capacitance. The power electronics supplied with the cryocooler, which is proprietary, allows setting the desired cold tip temperature or the input voltage to the compressor. In all our experiments the input voltage to the compressor is set to a constant value. The temperature of the cold heat exchanger is measured using a silicon diode sensor, which has a maximum error of $\pm 0.5 \mathrm{~K}$ for the temperature range of $54 \mathrm{~K}$ to $300 \mathrm{~K}$. An electrical resistance of $22 \Omega$ is used to input heat at the cold heat exchanger. The regenerator and the cold heat exchanger are made up of stainless steel - 304 (SS304) and copper material, respectively, for which temperature-dependent thermal properties are shown in Figure 7.1. The photos of the experimental setup together with a schematic representation of the cryocooler cold finger are shown in Figure 7.2. The length $(L)$ and diameter $(D)$ of the cold finger are $80 \mathrm{~mm}$ and $25.2 \mathrm{~mm}$, respectively. The cold tip is wrapped with multi layer insulation. The ventilators maintain a temperature of less than $310 \mathrm{~K}$ 
when the cooler is in operation at full power to the compressor, which is equal to $180 \mathrm{~W}$ in our case.

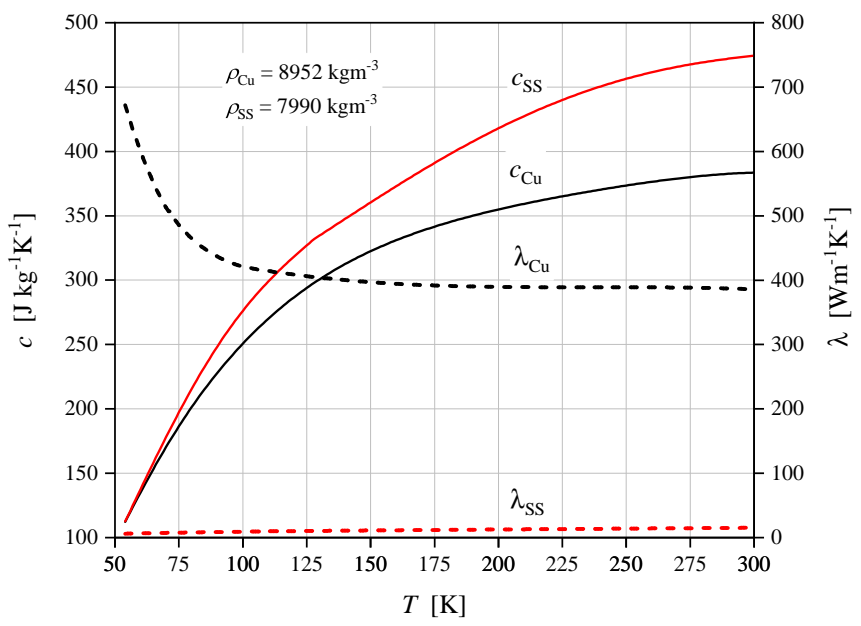

Figure (7.1) Specific heat and thermal conductivity of copper and stainless steel (SS 304) material [86-88]
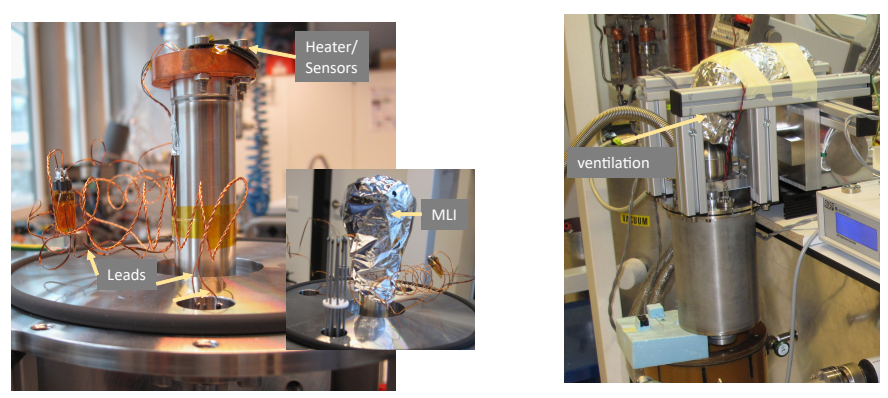

$$
T=T_{\infty} \quad-\lambda A_{\text {eff }} \frac{\partial T}{\partial x}=m_{\mathcal{c}} c_{\mathcal{C}}\left(T_{c}\right) \frac{d T_{c}}{d t}
$$

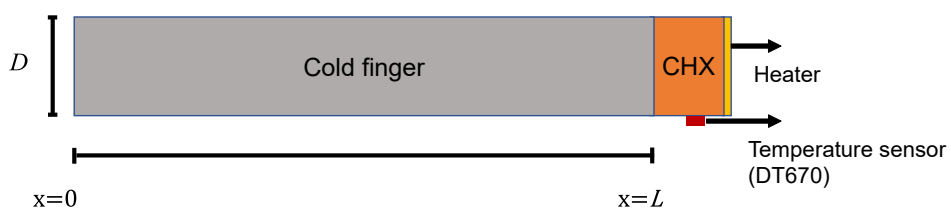

Figure (7.2) (above) Photos of the experimental set up. (below) Schematic representation of the cold finger. Here, $L=80 \mathrm{~mm}$ and $D=25.2 \mathrm{~mm}$ 
All the measurements are performed with the cold end facing down. The experiments performed are summarized in Table 7.1, and the results are discussed in the next section. To demonstrate the repeatability, at least three independent experimental runs were performed for each measurement.

\begin{tabular}{l|l|l|l|l}
\hline $\begin{array}{l}\text { Exp. } \\
\text { no. }\end{array}$ & $\begin{array}{l}\text { Initial state } \\
\text { of the cooler }\end{array}$ & $\begin{array}{l}\text { Heater } \\
\text { input }\end{array}$ & Compressor & Objective \\
\hline 1 & $\begin{array}{l}\text { Cooled to } \\
\text { no-load temper- } \\
\text { ature }\end{array}$ & $\begin{array}{l}\text { pulse, 23 W, } \\
15 \text { seconds }\end{array}$ & OFF & $\begin{array}{l}\text { Determine intrinsic } \\
\text { cold mass }\end{array}$ \\
\hline 2 & $\begin{array}{l}\text { Room tempera- } \\
\text { ture }\end{array}$ & $0,2,4 \mathrm{~W}$ & ON & $\begin{array}{l}\text { Transient cool- } \\
\text { ing power during } \\
\text { cooldown }\end{array}$ \\
\hline 3 & $\begin{array}{l}\text { Set cold temper- } \\
\text { ature }\end{array}$ & $\begin{array}{l}\text { several val- } \\
\text { ues }\end{array}$ & ON & $\begin{array}{l}\text { Steady state cool- } \\
\text { ing power }\end{array}$ \\
\hline 4 & $\begin{array}{l}\text { Cooled to } \\
\text { no-load temper- } \\
\text { ature }\end{array}$ & $\begin{array}{l}2,4,8,10.8 \\
\mathrm{~W}\end{array}$ & ON & $\begin{array}{l}\text { Transient cool- } \\
\text { ing power during } \\
\text { warmup }\end{array}$ \\
\hline 5 & $\begin{array}{l}\text { Cooled to } \\
\text { no-load temper- } \\
\text { ature }\end{array}$ & $0,2 \mathrm{~W}$ & OFF & $\begin{array}{l}\text { Effective cold fin- } \\
\text { ger cross-sectional } \\
\text { area }\end{array}$ \\
\hline 6 & $\begin{array}{l}\text { Room tempera- } \\
\text { ture }\end{array}$ & - & $\begin{array}{l}\text { Cooling of a 1.25 } \\
\text { kg copper mass at- } \\
\text { tached to the cold } \\
\text { heat exchanger }\end{array}$ \\
\hline
\end{tabular}

Table (7.1) List of experiments performed with the pulse tube cryocooler

\subsection{Results and discussion}

\subsubsection{Effective cold mass at the cold heat exchanger}

The heater attached to the cold heat exchanger is triggered with a heat pulse of duration $15 \mathrm{~s}$ and an electrical power $Q_{h}$ of $23 \mathrm{~W}$. The heat diffusion time into the regenerator $t_{d} \sim\left(L^{2} / \alpha\right)$ is $10^{3} \mathrm{~s}$, which is two orders of magnitude larger than the heat pulse duration. Therefore, to a good approximation, the heat diffusion to the regenerator may be neglected; this approximation is verified later in the subsection 7.3.3. The temperature increase of the temperature sensor 
attached to the cold heat exchanger is shown in Figure 7.3. The temperature derivative is obtained by fitting a curve to the temperature data. The effective cold mass is determined using,

$$
Q_{h}=m_{c} c_{c}\left(T_{c}\right) \frac{d T_{c}}{d t}
$$

where $c_{c}$ is the specific heat capacity of copper. The derived effective mass $m_{c}$ is also shown in Figure 7.3. The data is fairly constant in the duration of the pulse except at the initial and final parts of the pulse, which is due to the limitation of the power supply, namely, the slew rate of the control electronics. The effective cold heat exchanger mass determined using this method is in the range of $130-135 \mathrm{~g}$.

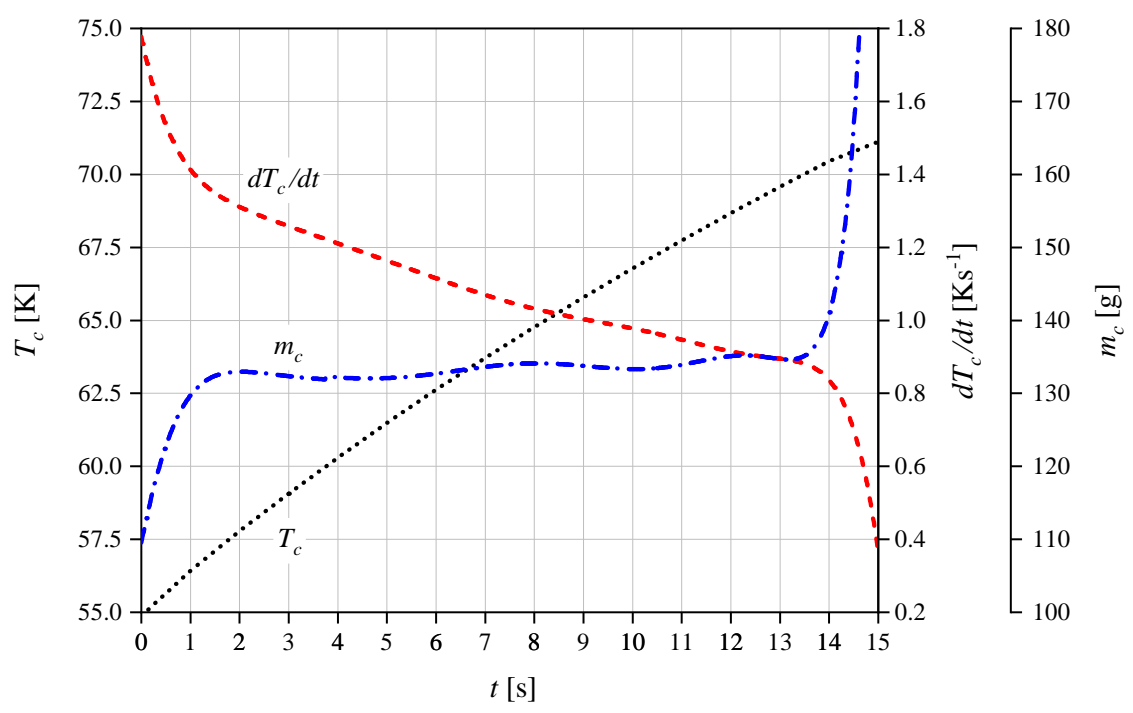

Figure (7.3) Cold heat exchanger temperature $T_{c}$ increase, its temporal derivative $\frac{d T_{c}}{d t}$ and estimated values of the effective copper mass $m_{c}$ in heat pulse experiments with the cryocooler.

\subsubsection{Steady and transient cooling power}

The cooling power available at the cold heat exchanger is discussed in this section for two cases, namely 'steady' and 'transient'. In the steady situation, 
the cooler is cooled to its lowest attainable temperature, after which a controlled heat load is applied using an electric heater mounted on the cold heat exchanger. The cooler warms up to a steady temperature, which is recorded. The measured steady-state cooling power $\left(Q^{s}\right)$ at five cold heat exchanger temperatures are shown in Figure 7.4.

(a)

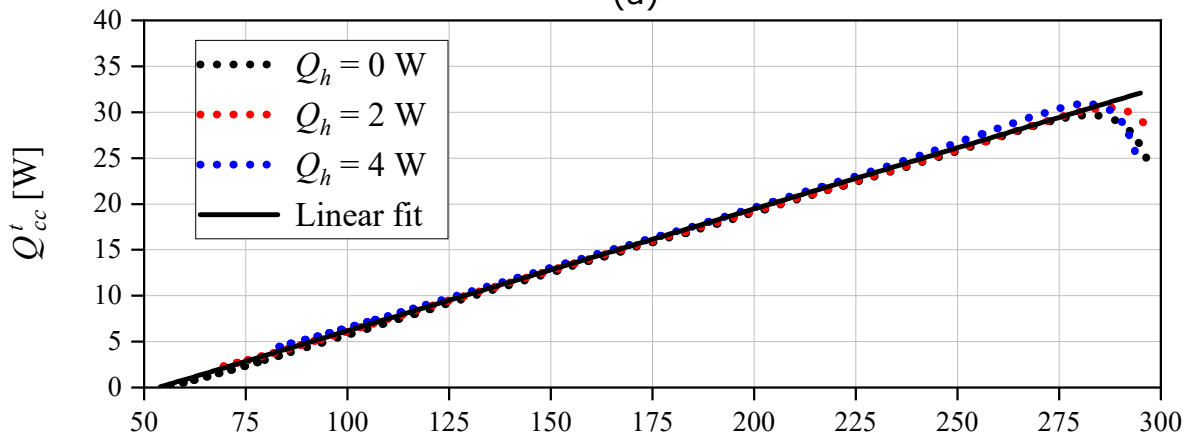

(b)

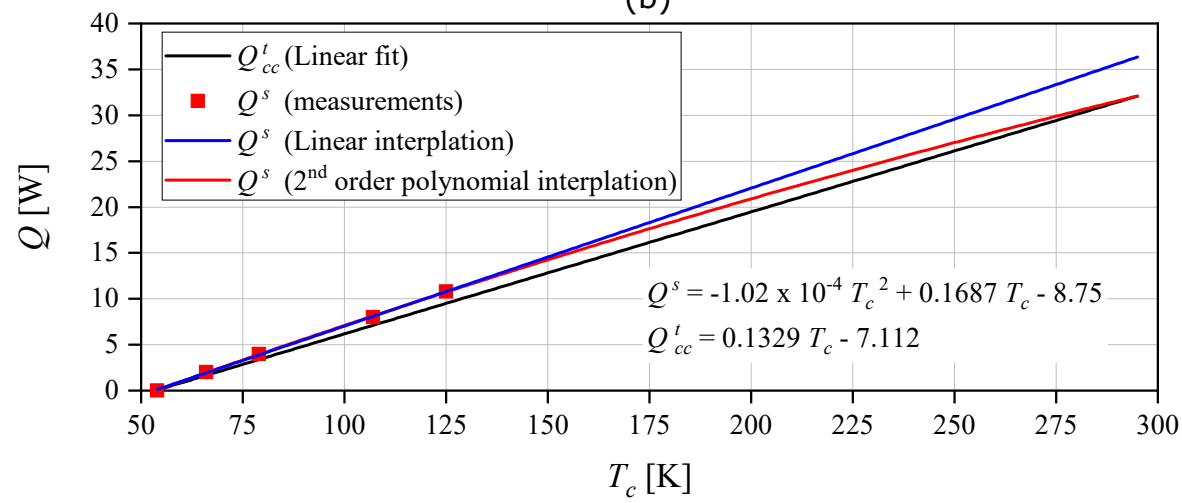

Figure (7.4) Steady-state (a) and transient (b) cooling power values as functions of the cold-end temperature.

The transient cooling power is determined in two scenarios, during cooldown and warm-up of the cooler. During cool-down the compressor input voltage is maintained at a constant value, and during warm-up, the compressor is turned off. The measured cold heat exchanger temperatures values during cool-down with a steady heating power of $0 \mathrm{~W}, 2 \mathrm{~W}$, and $4 \mathrm{~W}$ with a constant compressor power are shown in Figure 7.5. In the same figure, the temperature-time derivative is also shown. The derivative is obtained from a curve fitted to the temperature-time data. The cooling power $Q_{c c}^{t}$ is deter- 
mined from Equation 7.1; in this case, the cold mass $m_{c}$ of $130 \mathrm{~g}$ determined above is used. Figure 7.4 (a) shows the transient cooling power as a function of temperature, which is fairly linear. The dip seen in the data points at room temperature is similar to the observation by Grossman et al. [83], which could be due to the start-up of the cooler. However, due to a lack of instrumentation in our commercial cooler, we cannot ascertain the reason for this behavior. A fit for all the data points for the transient cooling power values shows a linear trend (see Figure 7.4 (a)). Extrapolating the transient cooling power fit to room temperature we could obtain the expected cooling power for the transient case.

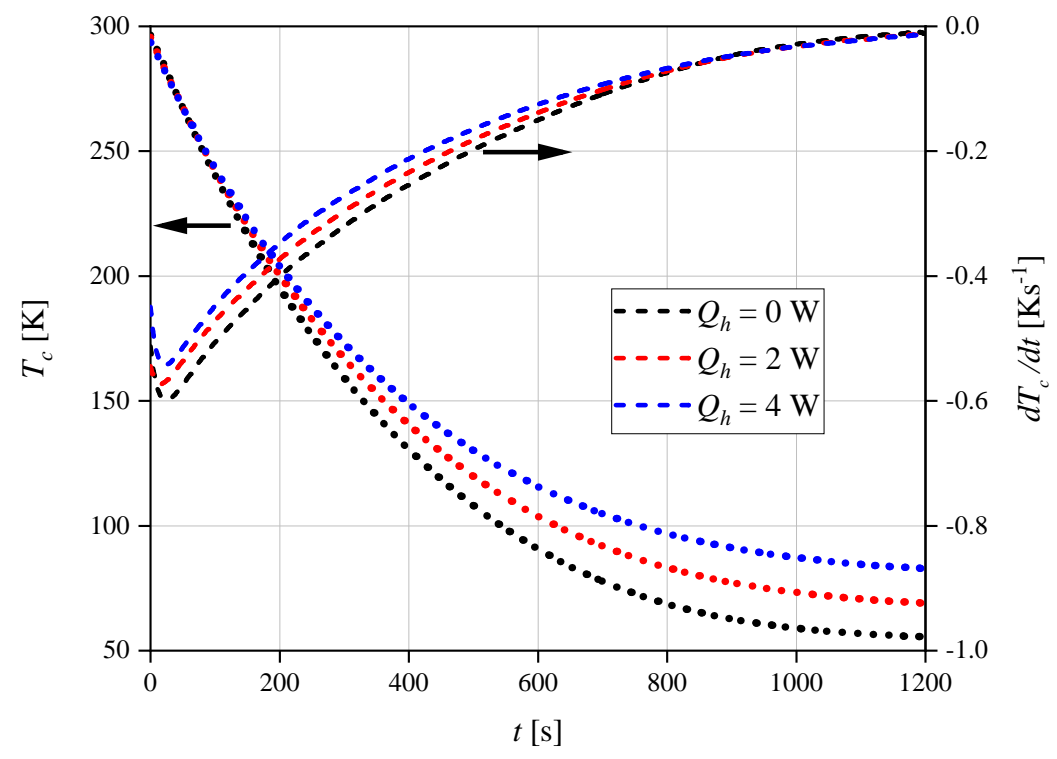

Figure (7.5) Cold heat exchanger temperature $T_{c}$ variation and its temporal derivative $\frac{d T_{c}}{d t}$ during cool-down experiments performed with different heat load $Q_{h}$ values at the cold heat exchanger.

The transient cooling power during cool-down is lower than the steady-state values for a corresponding cold temperature. This trend is expected because during cool-down the temperature profiles develop in the regenerator from the cold end towards the warm end, resulting in a higher temperature gradient at the cold-end during transient cool-down and thus higher heat leak to the 
cold-end. A linear fit to the measured, steady-state cooling power data is also shown in Figure 7.4 (b). At room temperature, the steady and transient curves should meet, which is not the case if a linear interpolation is assumed for the measured steady-state cooling power values. Using the measured cooling power values and the transient cooling power value at the room temperature, a second-order polynomial fit is obtained as the best fit for all data points. The reduced steady-state cooling power at lower temperatures is expected due to the non-linear effects in the regenerator material.

In an earlier work by Grossman et al. [83], the data presented in Figure 8 in their publication, shows an opposite trend, where the transient cooler power is larger than the steady-state cooling power. In their work, a temperature averaged specific heat capacity of copper is used to compute the heat capacity of the cold heat exchanger. However, the specific heat capacity decreases rapidly at low temperature (see Figure 7.2). Their data is further analyzed here to determine the effect of including a temperature-dependent heat capacity on the outcome of their work. The temperature-time data points are extracted using image analysis followed by the interpolation of extracted data points. A four-term Fourier series interpolation fits all the curves very well. The built-in mass (copper, stainless steel flanges, and screws) of the cold heat exchanger was specified by author as $26.40 \mathrm{~g}$, and the excess copper masses used for the experiments are $9.18 \mathrm{~g}, 42.10 \mathrm{~g}, 51.32 \mathrm{~g}, 83.73 \mathrm{~g}$, and $92.91 \mathrm{~g}$. The weights for the stainless steel flanges and screws in the cold heat exchanger are not mentioned, and therefore for simplification, the cold heat exchanger is assumed to be made of only copper. The calculated transient cooling power $Q_{c c}^{t}$ values during cool-down for all the six cases are now compared with the measured steady-state cooling power $Q^{s}$ values in Figure 7.6. The shape of the transient cooling power curve for all the six cases differs from that reported by Grossman et al. [83] and is very similar to the shape of the $Q^{s}$ curve. In addition, similar to the results presented in Figure 7.4 (a), the $Q_{c c}^{t}$ values for all six cases are slightly lower than the measured $Q^{s}$ values. The difference between the $Q_{c c}^{t}$ values among six cases is expected to be due to the limited number of data points and also due to the inaccuracy associated with the data extraction using the image analysis, resulting in slightly in-accurate interpolation for the temperature-time curve, especially at temperature values close to the room temperature. 
The transient cooling curves derived in this paper show a linear dependence, whereas those derived from Grossmann et al. [83] are not linear, which could be due to how the experiments are performed. In our case, the compressor power is kept constant, whereas, in the other work, the pressure ratio is kept constant. Nevertheless, using a simple experimental technique of a pulse heating experiment, followed by a cool down experiment, we demonstrated a method to determine the transient cooling power of a commercial cooler where the dimensions of the cold heat exchanger are unknown.

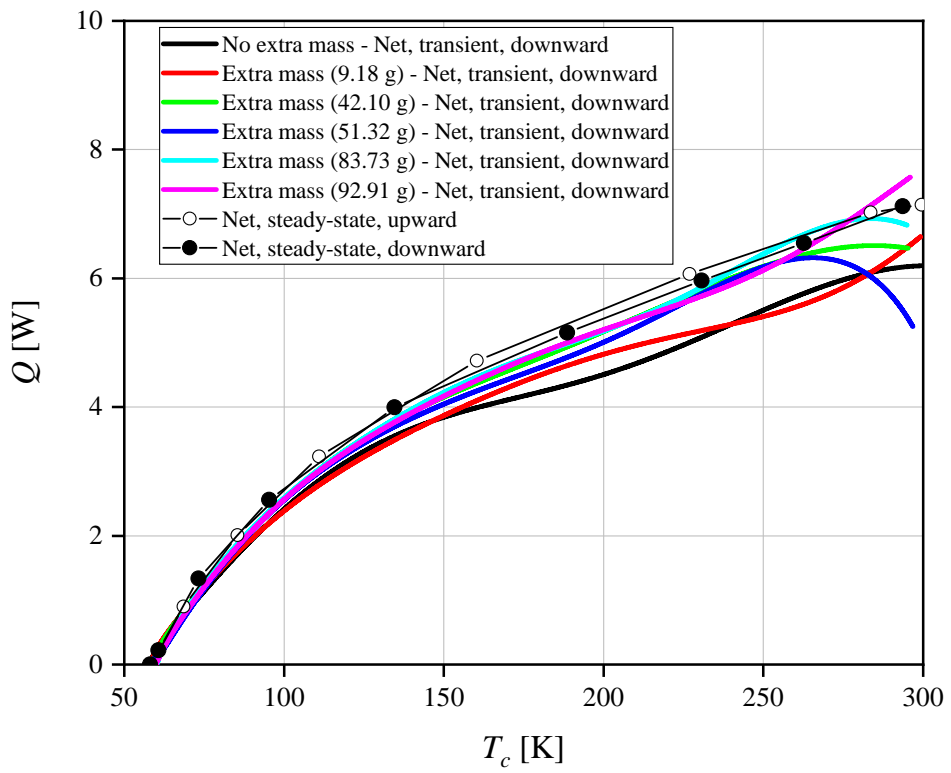

Figure (7.6) Transient cooling power values calculated from the temperature-time data reported by Grossmann et al. [83] are compared with the the corresponding steady-state cooling power values.

To calculate the transient cooling power during warm-up $Q_{c w}^{t}$, experiments are performed for different values of the external heat load $Q_{h}$ at the cold end and with the compressor turned on. The net transient cooling power during the warm-up is calculated using Equation 7.1. The temperature-time measurements from the experiments are shown in Figure 7.7a and the calculated transient cooling power curves are shown in Figure 7.7b. It can be seen that 
the transient cooling power values during the warm-up experiments are different from the cool-down experiments and also are slightly higher compared to the net steady-state cooling power values. The higher $Q_{c w}^{t}$ values are suspected due to lower parasitic heat load values compared to the steady-state conditions. For $Q_{h}=2 \mathrm{~W}$, the warm-up rate is lowest. This would give more time for the heating front from the warm end to diffuse towards the cold end of the regenerator and, therefore, would result in the temperature profile, which is closest to the steady-state conditions. Now, for higher $Q_{h}$ values, the warmup rate of the cold heat exchanger is higher, and therefore, the diffusion time for the heating front to move towards the cold-end of the regenerator will be lower. This would result in slightly lower temperature values close to the regenerator's warm end than in the steady-state case and, therefore, lower parasitic heat load values. In conclusion, the temperature profiles inside the regenerator during the transient cool-down/warm-up experiments are different from the steady-state case, resulting in different parasitic heat loads.

\subsubsection{Background heat losses}

In this section, a procedure to determine the background losses or parasitic heat leak is explained. It must be noted that in this discussion, only heat conduction through the regenerator is accounted for, which is a major source of heat leak. First, heat leak for steady-state conditions $Q_{p}^{s}$ is derived, followed by heat leak during the transient cool-down $Q_{p c}^{t}$ of the cryocooler.

\section{a) Steady state heat leak}

The heat flux through the regenerator $Q^{\prime \prime}\left(T_{c}\right)$ spanning a temperature of $T_{\infty}$ and $T_{c}$ is given by,

$$
Q^{\prime \prime}\left(T_{c}\right)=\frac{1}{L} \int_{T_{c}}^{T_{\infty}} \lambda(T) d T
$$

Based on the thermal conductivity of the stainless steel 304 [88], the length of the regenerator, in our case is $80 \mathrm{~mm}$, resulting in a heat flux value of $3.25 \times 10^{4} \mathrm{Wm}^{-2}$ for $T_{c}=80 \mathrm{~K}$ and $T_{\infty}=295 \mathrm{~K}$. 


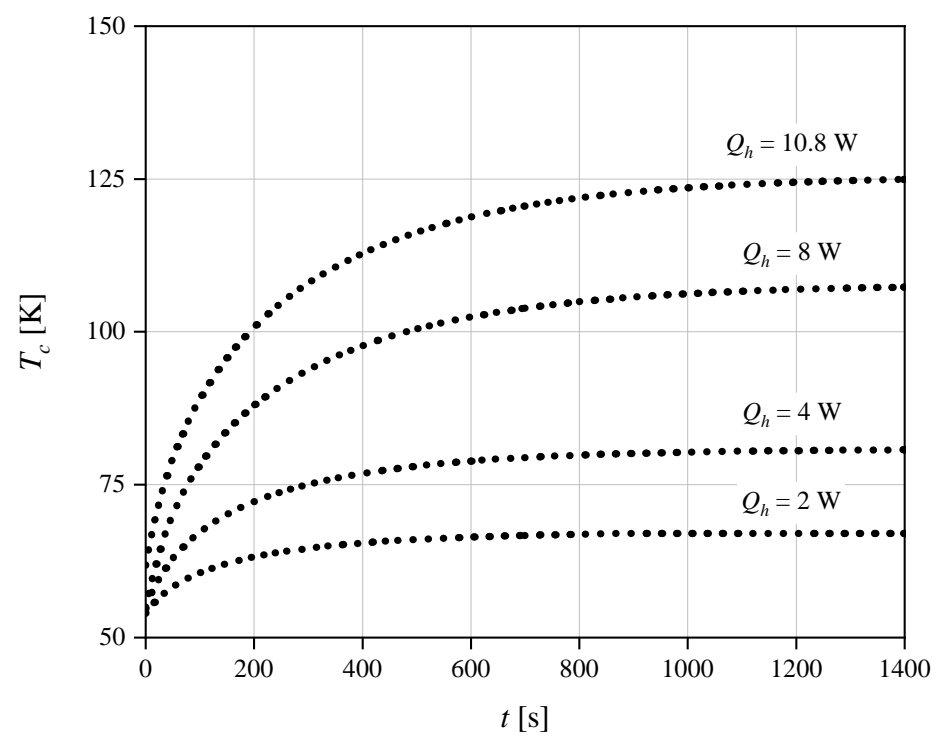

(a) $\mathrm{CHX}$ temperature $\left(T_{c}\right)$

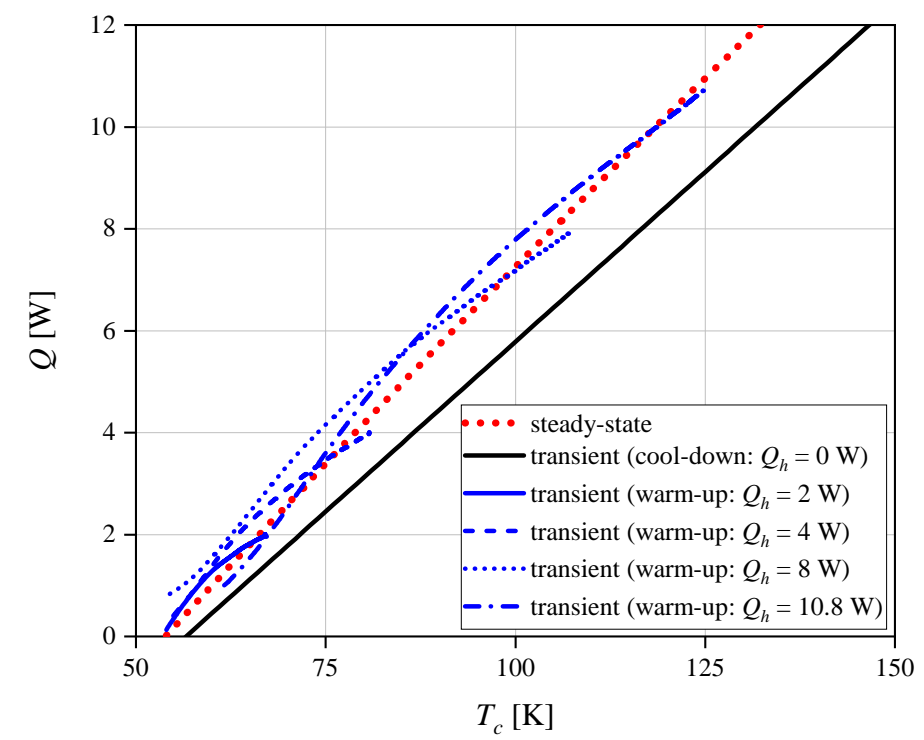

(b) Net cooling power

Figure (7.7) Cold heat exchanger temperature $T_{c}$ variation and the calculated cooling power values from the experiments performed with different heat load $Q_{h}$ values at the cold heat exchanger. 
To determine the heat leak $Q_{p}^{s}$, the effective cross-sectional area $A_{\text {eff }}$ should be known. The procedure adopted to determine the effective area is as follows; First, the cooler is allowed to attain a steady-state cold temperature. The electric power to the compressor is turned off, and the cold heat exchanger temperature is measured during warm-up. In the second set of experiments, also a constant heat is supplied by a heater equal to $2 \mathrm{~W}$. Figure 7.8 shows the temperature rise in both cases. In the first minute during the warm-up, the temperature rise is less than $25 \mathrm{~K}$ from the steady-state value. We assume that the regenerator temperature profiles during this short time scale are not much different from the steady-state situation.

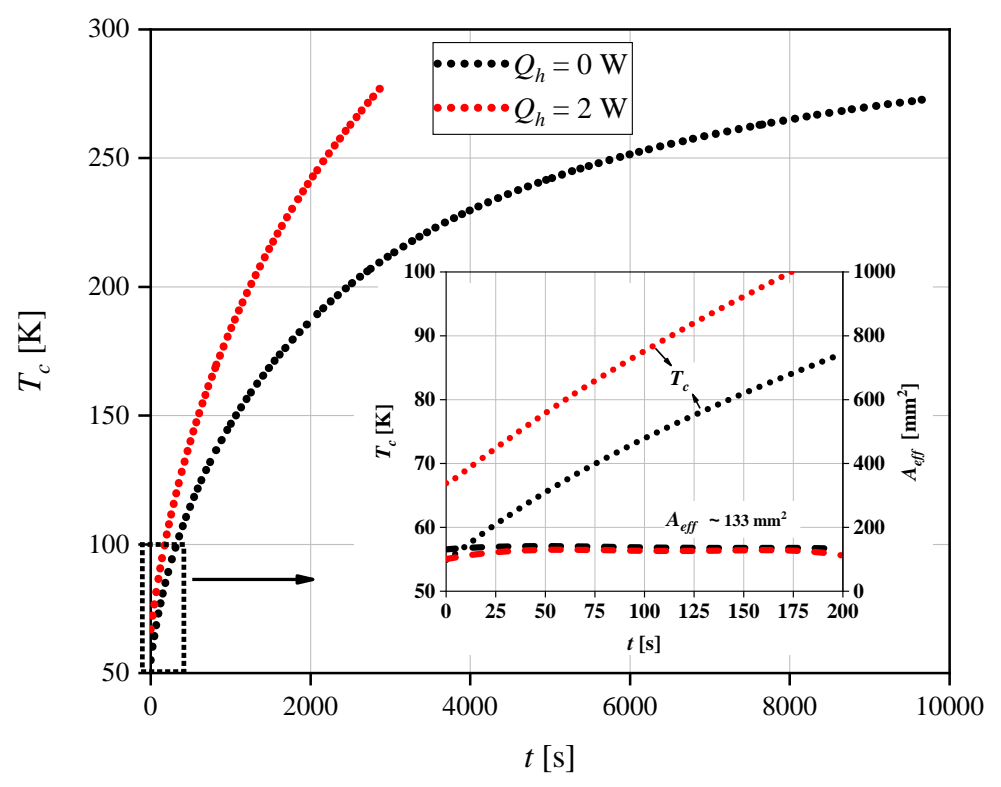

Figure (7.8) Temperature variation of the cold heat exchanger during warmup experiments and the values of the effective heat transfer area $A_{\text {eff }}$ calculated using Equation 7.3 
Following this, we can write,

$$
Q_{p}^{s}\left(T_{c}\right)=Q^{\prime \prime}\left(T_{c}\right) A_{e f f}=m_{c} c_{c}\left(T_{c}\right) \frac{d T_{c}}{d t}-Q_{h}
$$

where $Q_{h}=0$ and $2 \mathrm{~W}$ for the two cases. The effective cross-sectional area is calculated from this equation, which is also shown in the snippet of Figure 7.8. The effective area of $133 \mathrm{~mm}^{2}$ is $21 \%$ of the cold end cross-sectional area. This value is reasonable, given the reduced area due to the pulse tube and the porosity of the regenerator. The calculated value of the effective area also includes the coefficient for the reduced thermal conductivity value of the regenerator mesh. The steady-state parasitic heat leak $Q_{p}^{s}$ as a function of cold heat exchanger temperature is shown in Figure 7.9. The gross cooling power obtained as a sum of the parasitic heat leak and the net cooling power is also shown in the Figure 7.9.

The heat capacity of the part of the regenerator through which the heat pulse diffuses during the heat pulse experiment (Exp. no 1 in Table 7.1) is now calculated using $C_{\text {reg }}=\rho c_{\text {reg }} A_{e f f} L_{d}=1.23 \mathrm{JK}^{-1}$. In this, the thermal diffusion length is estimated using, $L_{d} \approx \sqrt{\alpha t_{d}} \approx 1 \mathrm{~cm}$. The calculated regenerator heat capacity $\left(C_{r e g_{d}}\right)$ is approximately $8 \%$ of the heat capacity of the cold end heat exchanger and therefore our assumption of neglecting the heat absorbed by the regenerator material during the heat pulse experiment is valid.

\section{b) Transient heat leak}

The gross cooling power of a pulse tube cryocooler is the product of the dynamic pressure amplitude, volume flow, and the phase difference between these. We assume that for a particular cold-tip temperature, the gross cooling is not influenced by the temperature profiles in the regenerator. Therefore, the gross cooling calculated for the steady case will be the same as for the transient case. The net transient cooling power during the cool-down $Q_{c c}^{t}$ is obtained earlier. The parasitic heat leak is the difference between the gross cooling power and the net transient cooling power shown in Figure 7.9. The parasitic heat leak is higher in the transient operation compared to the steadystate as expected due to larger temperature gradients in the regenerator, which is derived next. 


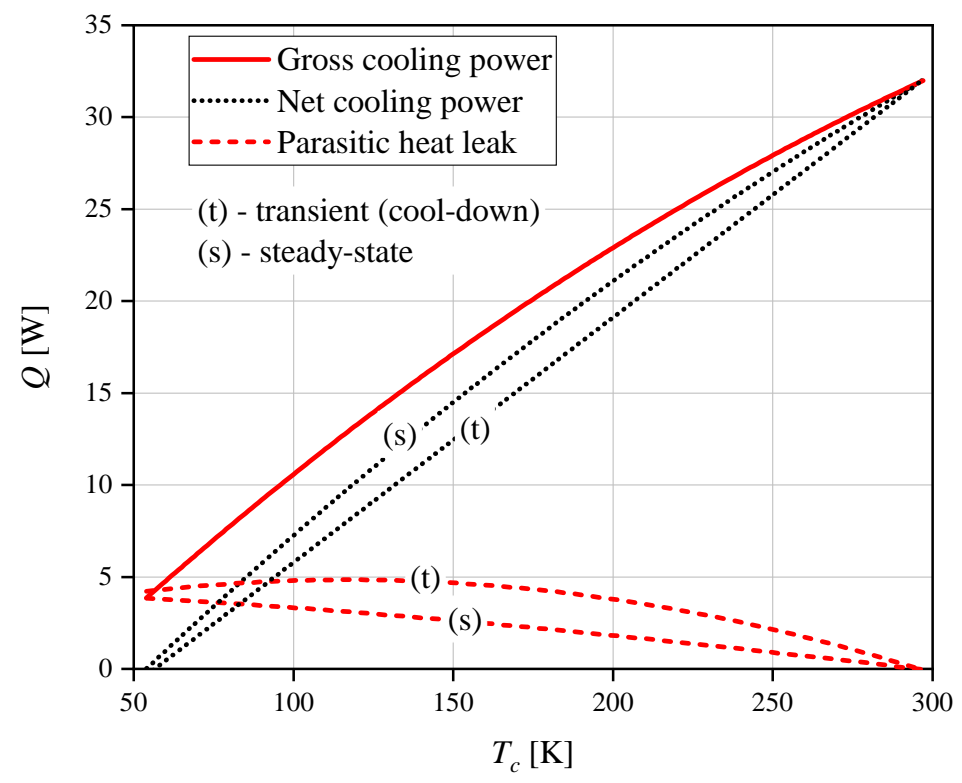

Figure (7.9) Parasitic heat leak and cooling power values as a function of cold heat exchanger temperature

\section{Temperature profiles in the regenerator}

Assuming a one-dimensional temperature distribution in the regenerator, the temperature profile along the regenerator's length for different cold-tip temperatures can be obtained from the parasitic heat leak calculated above. The temperature at the warm end of the cooler is $T_{\infty}$, and at the cold side, the temperature is $T_{c}$. Using these temperature boundary conditions and the heat leak for steady-state $Q_{p}\left(T_{c}\right)$ and transient conditions $Q_{p c}^{t}\left(T_{c}\right)$, the temperature at any location along the length of the regenerator is derived using,

$$
-A_{e f f} \int_{T_{\infty}}^{T} \lambda(T) d T=Q_{p}\left(T_{c}\right) \int_{0}^{x} d x
$$

Here, temperature dependent thermal conductivity values of SS 304 are used, which are given by [88], $\lambda(T)=-3.988389 \times 10^{-9} T^{4}+3.411681 \times$ $10^{-6} T^{3}-1.088656 \times 10^{-3} T^{2}+1.813807 \times 10^{-1} T-1.031521$, for the temperature range of $45 \mathrm{~K}$ to $293 \mathrm{~K}$. The calculated steady-state temperature 
profiles are shown in the Figure 7.10, which appear non-linear due to the temperature-dependent thermal conductivity of the regenerator material. To determine the temperature profile during the transient cool-down, a cubic temperature profile inside the regenerator is considered, $T(x)=a+b x+c x^{2}+d x^{3}$. A cubic polynomial is chosen due to the limited boundary conditions, which are shown below.

$$
\begin{gathered}
T(x=0)=T_{\infty} \\
\left.\left(T(x=0)=T_{\infty}\right) \Longrightarrow \frac{\partial T}{\partial t}\right|_{x=0}=\left.\frac{\partial^{2} T}{\partial x^{2}}\right|_{x=0}=0 \\
T(L)=T_{c} \\
-\lambda\left(T_{c}\right) A_{e f f} \frac{\partial T}{\partial x_{x=L}}=Q_{c c}^{t}\left(T_{c}\right)
\end{gathered}
$$

For each setting of the cold temperature $T_{c}$ the solution to the temperature profile is shown in Figure 7.10. Comparing the steady-state and transient temperature profiles, the gradient at the cold-end in case of the transient case is larger than the steady case as one would expect.

It must be noted here that the warm-end of the cryocooler in the above discussion of background losses is taken as a constant value, $T_{\infty}=295 \mathrm{~K}$. In reality, during the cool down, the warm-end rises in temperature and in our particular case to a maximum value of $310 \mathrm{~K}$. To determine the impact of this temperature rise, we estimated the background losses using the procedure discussed above for $T_{\infty}=295 \mathrm{~K}$ and $310 \mathrm{~K}$. The difference in the heat leak value is less than $0.25 \mathrm{~mW}$. This data however, does not influence the estimation of transient or steady cooling power as the cold end temperature is used to determine these parameters. 


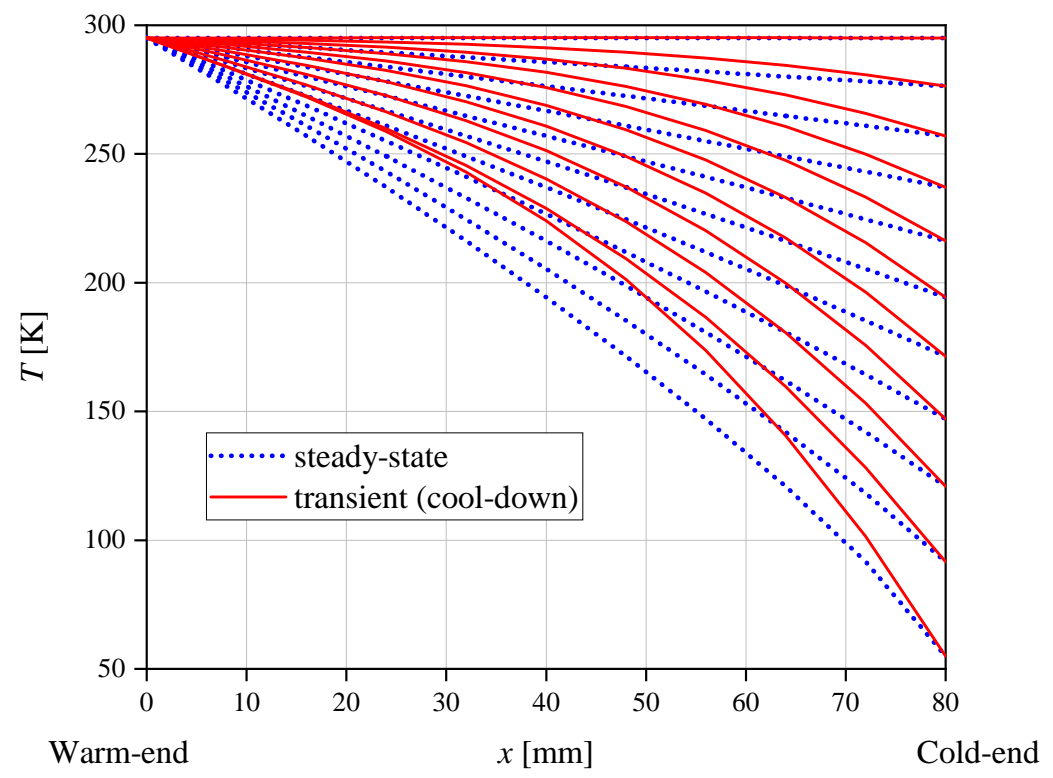

Figure (7.10) Calculated temperature variation inside the regenerator during transient cool-down and steady-state.

\subsubsection{Cool-down of an added mass to the cold-end}

A copper mass of $1.25 \mathrm{~kg}$ is attached to the cold heat exchanger of the cryocooler with the aim to cool vials for snap-freezing tissues as discussed in chapter 5. Our aim is to predict the cool-down time of the cold mass as this is an important parameter to determine the preparation time of the system for subsequent snap-freezing procedures. Having determined the steady cooling power $Q_{c}^{s}$ and the transient cooling power $Q_{c c}^{t}$ in previous sections, we will now check which of these powers better predicts the cooling of the cold mass. The duration of the cooling from an initial temperature $T_{i}$ to a particular low temperature $T_{c}$ is,

$$
\int_{0}^{t} d t=\int_{T_{i}}^{T_{c}} m_{c} c_{c} \frac{d T_{c}}{Q_{n e t}\left(T_{c}\right)}
$$

where, $Q_{n e t}=Q_{c c}^{t}$ or $Q_{n e t}=Q^{s}$ (for values of $Q_{c c}^{t}$ and $Q^{s}$ see Figure 7.4). The temperature-time values for both cases are compared with the cool-down 
measurements in Figure 7.11. The temperature curve using $Q_{c c}^{t}$ values agrees very well with the measurements, whereas, the calculated values $Q_{n e t}$ using $Q^{s}$ show a significant difference from experimental data.
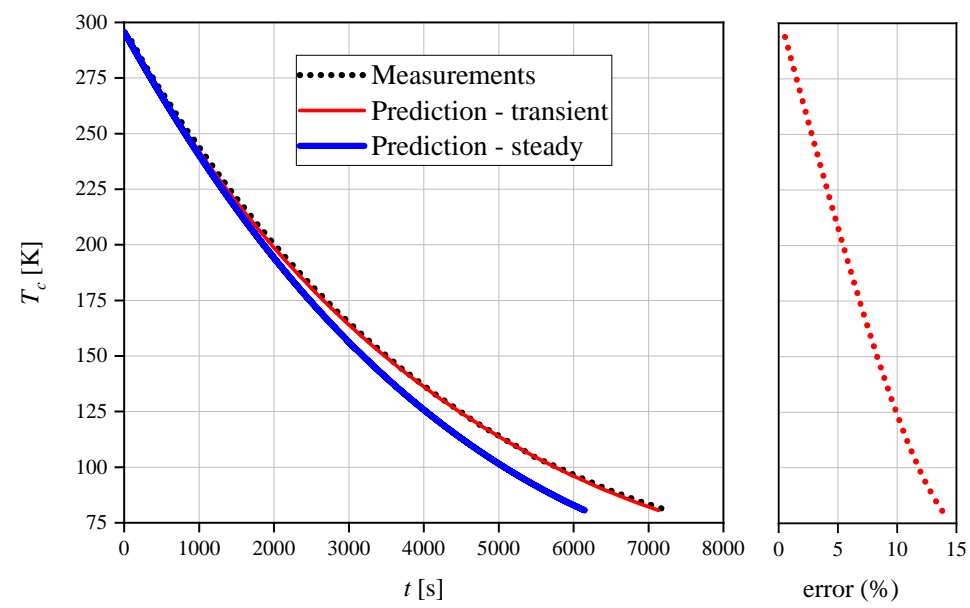

Figure (7.11) Measured and predicted temperature-time values during cooldown of a copper mass of $1.25 \mathrm{~kg}$ attached to the cold-end of the cryo-cooler. Error when using transient and steady cooling power $\left(\frac{t_{\text {transient }}-t_{\text {steady }}}{t_{\text {transient }}}\right)$, which is the difference between the cool-down time calculated using steady-state and transient cooling power values compared to transient case is shown.

In deriving the transient cooling power, we used Equation 7.1, which require as input the cold mass $m_{c}$. Therefore, the question is why the agreement of our prediction with experimental data is rather good in spite of a large cold mass $(1.25 \mathrm{~kg})$ attached to the cooler. To throw light on this issue let us consider the diffusion time inside the regenerator material, which is, $t_{d} \sim\left(L^{2} / \alpha\right) \sim 10^{3}$ s. Now, let us estimate the cooper mass for which the temperature drop $(\Delta T)$ in $10^{3}$ seconds is negligible. For calculations, we assumed $\Delta T=1 \mathrm{~K}$, heat capacity $200 \mathrm{~J} \cdot \mathrm{kg}^{-1} \cdot \mathrm{K}^{-1}$ at $80 \mathrm{~K}$ and a transient cooling power at $80 \mathrm{~K}$ equal to $2.86 \mathrm{~W}$. The calculated value of the copper mass is about 14 $\mathrm{kg}$. This value is an order in magnitude larger than the attached mass in our experiments. With such a large heat capacity, the regenerator temperature profiles will be similar for both steady-state and transient cases. This results in the same cooling power values for both cases. The maximum possible 
error value, which is the difference between the cool-down time calculated using steady-state and transient cooling power values compared to the transient case, is also shown in the Figure 7.11. The maximum error in predicting the cool-down time increases with the decrease in the cold-end temperature and is roughly $14 \%$ for a temperature of $80 \mathrm{~K}$.

\subsection{Conclusions}

We presented a systematic approach to determine key operational parameters of a commercial off-the-shelf pulse tube cryocooler.

- By releasing a short heat pulse on the cold heat exchanger, we make use of the fact that the diffusion time in the regenerator is much larger, and all the energy introduced is absorbed by the cold mass. From this experiment, the effective cold mass is determined.

- The temperature of the cold heat exchanger is measured during the cooldown of the cryocooler with and without constant heat input. The transient cooling power during cool-down is determined from the above derived cold mass, and the temperature-time derivate from these experiments. Note that the transient cooling power during cool-down is lower than the steady state cooling power.

- After attaining the lowest no-load temperature, steady heat input is applied to the cold heat exchanger causing the temperature at the cold heat exchanger to increase. The transient cooling power during the warm-up is determined following the sam e procedure as above. Note that the transient cooling power during warm-up is larger than the steady state cooling power.

- Experiments with a copper mass of $1.25 \mathrm{~kg}$ attached to the cooler. The temperature-time predicted using transient cooling power agrees well with the experiments whereas the steady cooling power does not. This is due to the fact that the thermal diffusion time in the regenerator is longer than the cool-down speed of the cold mass. When a large mass is attached, to give a figure of merit $14 / 2.86 \mathrm{~kg} . \mathrm{W}^{-1}$ mass to cooling power ratio, the cool-down of this heat capacity is of the same order of magnitude as the regenerator. In this case, steady state cooling power can be used to determine the cool-down dynamics. 
- We showed that parasitic heat leaks in the transient and steady state can be determined from warm-up experiments. The heat leak is a useful parameter to benchmark the performance of a cryocooler. 


\section{CHAPTER 8 \\ Conclusions and Outlook}

\subsection{Conclusions}

The thesis is divided into two distinct sections. In section-I, we concluded the thermal assessment of the standard tissue snap-freezing procedures and also developed a phenomenological understanding of the heat transfer at the vialliquid interface and inside the vial wall. In section-II, we discussed the design process of an electrically operated tissue snap-freezer. We also presented a heat transfer model to predict the vial cool-down in the snap-freezer. The preparation time for the snap-freezer requires understanding of the transient cooling characteristics of the commercial off-the-shelf cryocooler used in the device, which is also discussed in section-II.

\section{Section-I}

Cooling experiments performed with the cryo-vials in liquid nitrogen and isopentane show some interesting outcomes: a polypropylene vial cools faster in the liquid nitrogen than in isopentane whereas the opposite is true for the case of an aluminum vial; the higher heat transfer coefficient caused by the early transition from film to the nucleate boiling regime for the polypropylene vial explains this result.

So far, cool-down prediction of the polypropylene vials in liquid nitrogen was an empirical approach due to the scientific challenges discussed in section 1.3. So, here we presented an analytical approach to predict the temperature variation inside the vial wall and also of the tissue attached to it during its cool-down in liquid nitrogen. To develop a generalized heat transfer model for this, we approximate the temperature-dependent specific heat of the polypropylene material to vary linearly with temperature and proved that the Perfect Thermal Contact Assumption (PTCA) at the liquid-solid interface is valid for the polypropylene material. A fairly good prediction of the tem- 
perature variation at the inner wall of an empty polypropylene tube is then obtained with a maximum error of less than $10 \%$ compared to measurements. The PTCA assumption assumes constant temperature at the liquid-solid interface and thus eliminate the need to evaluate the heat transfer coefficient at the liquid-solid interface. Also, a one dimensional approach used in the model, allow user to predict the temperature of the vials of any shape and size. The outcome from this model is that the difference in the cool-down time of a tissue in a tube and an empty tube is proportional to the product of the tissue heat capacity and the tube-wall thermal resistance.

A phenomenon similar to the polypropylene material in liquid nitrogen is also observed for a commonly used trick, where the cooling rate of a metal in saturated liquids like liquid nitrogen is improved by insulating its surface with a material that has the thermal effusivity value of at least two orders lower than the metal to be cooled. A detailed understanding of such behavior would require micro-scale study of the boiling phenomenon, which is outside of the scope of the work presented here. However, using the phenomenological understanding of the cool-down behavior for the polypropylene material in liquid nitrogen, which also has a thermal effusivity value two orders lower than metals like aluminum and copper, we developed an experimental approach, which will give cryogenic application engineers a predictive tool to design systems for improving the cool-down of metals in cryogenic liquid baths.

\section{Section-II}

To design the tissue snap-freezer, we assessed different cooling principles to meet the device requirements. Among all the principles, forced convective gas flow meets all the requirements and, therefore, is chosen as the cooling principle for the device. We also developed a heat transfer model to predict the temperature variation of a commercial aluminum vial cooled inside the snap-freezer. The results from the model are also verified with measurements performed using different snap-freezer settings such as different operating temperature, mass flow rate and gas thermal properties. We also discussed the increased cool-down effect caused due to misalignment of the vial with respect to the device axis. 
To study the transient characteristics of the commercial off-the-shelf pulse tube cryocooler used in the snap-freezer, we presented an experimental approach that allowed us to determine the key operational parameters of the cryocooler. In contrast to previously reported studies, we found that the transient cooling power values during cool-down are lower than the steady-state cooling power, whereas during warm up it is higher than the steady-state cooling power. The reason for such behavior is suspected to be caused by a difference in the regenerator temperature profiles during cool-down and warm-up, resulting in different parasitic heat leaks to the cold end of the cooler. We also validated the calculated transient cooling power values during cool-down using cool-down measurements performed with a copper mass of $1.25 \mathrm{~kg}$ attached to the cooler.

To summarize, the work presented in this thesis is a significant contribution to understanding the cooling dynamics of the conventional snap-freezing procedures, which will be helpful in improving and standardizing the conventional tissue snap-freezing procedures. We also developed a tissue snapfreezing device to provide an alternative to the existing procedures. This device reducing the allows controlled freezing of the tissue enclosed in a commercial cryo-vial and, therefore, will be helpful in accelerating the research on how the cooling rate effects the frozen tissue quality. The developed device does not release any harmful cryogens and, therefore, is safe to use inside the operation theater. By doing so, it reduces the time between tissue acquisition and freezing and thus will be very helpful in preserving the quality of the frozen tissue.

\subsection{Outlook}

\section{Next generation snap-freezer to include cold transportation:}

The tissue workflow during biopsy involves tissue/cells acquisition, snapfreezing, cold transport and diagnosis [13] as shown in the Figure 8.1. In the project CryoOn, we focused on improving the snap-freezing part of the tissue work flow and therefore introduced an electrically operated snap-freezer [78] that introduces an opportunity for the snap-freezing process to occur inside the operation theater. This reduces the time-lag between the tissue acquisition and the snap-freezing, which may result in a better quality frozen tissue and 
standardizing the tissue workflow. However, to preserve the morphology of the frozen tissue/cells, its temperature should stay below the glass transition temperature of the water $\left(-137^{\circ} \mathrm{C}\right)$ [89] through out the entire biopsy procedure i.e. from its acquisition to the diagnosis. Therefore, cold transportation of the frozen tissue is of equal importance to preserve its quality.

The current practice of cold transportation at such a temperature involves usage of liquid nitrogen based shippers [90,91]. These shippers need a continuous availability of liquid nitrogen, which therefore requires managing large infrastructure and trained personnel for its maintenance. Additionally, these shippers release gaseous nitrogen into the atmosphere and, therefore, requires following several safety protocols. The liquid nitrogen based shippers also use vacuum based insulation, which deteriorates over time and thus requires a good quality check before each use. The realization of a compact system that snap-freezes a biopsy and also allows a stand-alone cold transport without using sacrificial cryogens would be a great contribution in improving the existing tissue work flow.

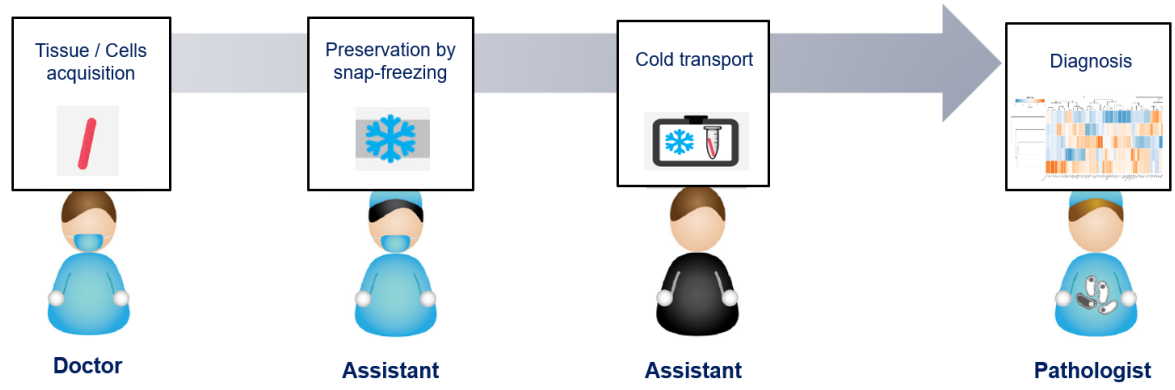

Figure (8.1) Tissue work flow during biopsy

The scientific research challenges for the development of such devices are in the development of novel insulating materials and innovative temperature stabilization techniques. A smart thermal insulation can be composed of hollow glass bubbles/aerogels or a foam arranged in a sandwich structure combined with layers of materials exhibit a solid to liquid phase transition. These materials exhibit a high latent heat at their phase transitions and can be used as a heat sink to replace the copper mass, which is used as a thermal energy storage unit in the snap-freezer discussed in this thesis. 
Such a Phase Change Material (PCM) can be used as a Thermal Energy Storage Unit (TESU) by enclosing it in a metallic container made up of high conductivity material such as copper, which is then connected to a cold source. Similar to the developed snap-freezer, the cryocooler can be used as a cold source to cool the PCM based TESU. To cool the vial in the TESU, a space for the vial insertion similar to the developed snap-freezer can be made and a static gas can then be injected in the gas-gap to transport the heat from the vial to the TESU. The advantage of using a PCM based TESU is that after cooling the cryo-vials, the same TESU can also be used in the cold transport of the vials. The high latent heat associated with the PCM can absorb the heat leaks from the surroundings in order to maintain a stable low operating temperature for the vials. The selection of materials and their design optimization is an important aspect in the design of such devices. For the temperature range of below $-150{ }^{\circ} \mathrm{C}$, isopentane is a potential candidate for the PCM, as it exhibits solid-liquid phase transition at approximately $-160^{\circ} \mathrm{C}$.

\section{Thermal Quenching of materials having low thermal effusivity:}

Although macroscopic aspects of the heat transfer at the solid-liquid interface and inside the solid material are discussed during cool-down of high and low conductivity materials in both saturated and sub-cooled liquids, a few questions still needs answers that would be helpful in enhancing our understanding of the thermal quenching in liquid nitrogen especially for materials with a thermal effusivity that is low compared to that of metals.

- What causes the earlier transition in the boiling regime for materials with a low thermal effusivity compared to metals?

- How does the surface temperature at the solid-liquid interface of materials having low thermal effusivity vary during its cool-down in a liquid nitrogen pool?

- What limits the critical heat flux values for insulated metals in liquid nitrogen? 


\section{Appendix}

\section{Bi-metallic cylinders quenched in liquid nitrogen:}

The usage of low conductive coatings to increase cooling speed of the metallic objects has been discussed in chapter 4. The coating thickness of $200 \mu \mathrm{m}$ reduces the cool-down time by a factor of roughly four times compared to its bare counter part. Although coating the high conductive materials with low effusivity materials is considered as an effective way for its increased cooldown, it also has its shortcomings; the cooling speed of the coated metals is very critical to the coating thickness value i.e. any alteration in the coating thickness via chemical reaction or scale deposition can significantly affect the cool-down of the objects to be cooled.

To overcome this, an alternate approach for which the reduced cool-down is dependent only on the surface temperature variation rather than the thermal resistance offered by the coating material has been investigated for the transient cool-down measurements. This is realized by producing a bi-metallic cylindrical substrate shown in the Figure 8.2a. The bi-conductive surfaces have a periodic arrangement of low-conductivity material embedded within a high-conductivity substrate. The thermal conductivity of the epoxy $(<1$ $\left.\mathrm{Wm}^{-1} \mathrm{~K}^{-1}\right)$ is several orders of magnitude less than that of copper $(\approx 400$ $\mathrm{Wm}^{-1} \mathrm{~K}^{-1}$ ). When submerged in liquid nitrogen, this creates an in-plane variation in surface temperature. Due to the extreme conductivity difference, there is negligible heat conducted through the epoxy divisions during nucleate boiling. As such, the wall superheat temperature above the epoxy divisions will be notably reduced as compared to that experienced over the copper sections. Due to low wall superheat, the epoxy-liquid interface will experience more liquid-solid contacts and thus help eliminating the vapor layer from the metal surface by channelizing the liquid flow over it; as observed in the steady-state measurements performed with flat bi-metallic plates in water by Rahman et al. [92]. From their measurement data, they also observed that the maximum increased heat transfer rate is obtained when the pitch value coincides with the capillary length of the water. The capillary length is a scaling factor that relates gravity and surface tension. It is a fundamental physical property that 
is found when body forces (gravity) and surface forces (Laplace pressure) are in equilibrium; it is given by,

$$
\lambda_{c}=\sqrt{\frac{\sigma}{g\left(\rho_{l}-\rho_{v}\right)}}
$$

in this, $\sigma$ and $\rho_{l}$ is the surface tension and density of the liquid. $g$ is acceleration due to gravity and $\rho_{v}$ is density of the vapor.

To fabricate these, grooves size with width and depth of $0.5 \mathrm{~mm}$ were first machined into copper cylinder of $60 \mathrm{~mm}$ length and $6 \mathrm{~mm}$ diameter, and subsequently filled with a low conductive epoxy (stycast 1266). After curing, the entire part was machined using a sand paper to remove excess epoxy, resulting in smooth surfaces with lines of epoxy material distributed along the cylinder into parallel strips. Bi-conductive cylinders were fabricated with a varying pitch $(P)$, which is the distance between the two epoxy strips. In total, four distinct surfaces have been fabricated and tested including a bare copper surface and three bi-conductive surfaces with $P$ values of $1.5,2.5$ and $4 \mathrm{~mm}$; such pitch values are chosen to keep them in the same order as the capillary length for liquid nitrogen, which is calculated using Equation 8.1 and is equal to $1.1 \mathrm{~mm}$.

The temperature of the cylinder is measured at its center using a type-E thermocouple, which is soldered at the inner end of the hole made at the center of the cylinder. The diameter and length of the hole is $1.2 \mathrm{~mm}$ and $3 \mathrm{~mm}$. The cylinder is immersed horizontally at room temperature and is maintained to approximately the same depth in the liquid nitrogen pool. The cylinder is held stationary during the measurements using a thin stainless steel tube of $1.2 \mathrm{~mm}$ outer diameter and $0.1 \mathrm{~mm}$ wall thickness glued to the side face of the cylinder. The length of the stainless steel tube inside the liquid pool is at approximately $10 \mathrm{~cm}$ for each experiment. Due to the low heat capacity of the tube, it cools faster compared to the cylinder and therefore the parasitic heat load through the tube is neglected in the present analysis. The large length of the tube inside the liquid pool also helps in further reducing the parasitic heat load through the tube. The measurements are recorded at the rate of 100 samples per second. To demonstrate the repeatability, three independent experimental runs were conducted for each measurement. Thermal properties of the stycast are assumed to be constant, its density $(\rho)$, specific heat capacity 
(c) and thermal conductivity $(\lambda)$ are $1120 \mathrm{kgm}^{-3}$ [60], $1000 \mathrm{Jkg}^{-1} \mathrm{~K}^{-1}$ [61] and $0.18 \mathrm{Wm}^{-1} \mathrm{~K}^{-1}$ [61], respectively. The specific heat capacity and thermal conductivity of the copper material is temperature dependent and are given by $c=\left(-1.35 \times 10^{-7} T^{4}+1.29 \times 10^{-4} T^{3}-4.73 \times 10^{-2} T^{2}+8.23 T-2.15 \times 10^{2}\right)$ $\mathrm{Jkg}^{-1} \mathrm{~K}^{-1}$ for $60 \mathrm{~K}<T<300 \mathrm{~K}$ [30] and $\lambda=\left(-1.26 \times 10^{-5} T^{3}+8.97 \times\right.$ $\left.10^{-3} T^{2}-2.11 T+5.55 \times 10^{2}\right) \mathrm{Wm}^{-1} \mathrm{~K}^{-1}$ [31] for $100 \mathrm{~K}<T<300 \mathrm{~K}$ respectively. The density of the copper is $8952 \mathrm{kgm}^{-3}$ [62].

The temperature-time measurements and the calculated cooling rate values for the bi-metallic substrates are shown in the Figure 8.2. The cool-down time for the bi-metallic cylinders is significantly lower compared to the bare copper cylinder and is roughly four time shorter for the pitch value of $1.5 \mathrm{~mm}$; the cylinder with $P$ value of $4 \mathrm{~mm}$ shows faster cool-down compared to the 2.5 $\mathrm{mm}$. The maximum cooling rate values for the bi-metallic cylinders are very high compared to its bare counter part. Similar to the bare cylinder, the cooling rate curve for the bi-metallic substrates also shows the conventional dome shaped curve for temperature lower than $100 \mathrm{~K}$, which is due to the change in boiling regime. However, in the temperature range higher than the $100 \mathrm{~K}$, all three bi-metallic substrates shows different trends; the cooling rates with 4 $\mathrm{mm}$ pitch value is decreasing with the reduction in metal temperature whereas, for pitch value of $2.5 \mathrm{~mm}$ a spike in the cooling rate is seen at approximately $200 \mathrm{~K}$. The cooling rate for the $1.5 \mathrm{~mm}$ pitch value increases from $300 \mathrm{~K}$ to $200 \mathrm{~K}$ and decreases afterwards till $100 \mathrm{~K}$. These outcomes of the preliminary experiments performed are interesting and are not understood at the moment. A detailed microscopic understanding of the phenomenon would be helpful in optimizing dimensions of such substrates to obtain faster cool-down. 


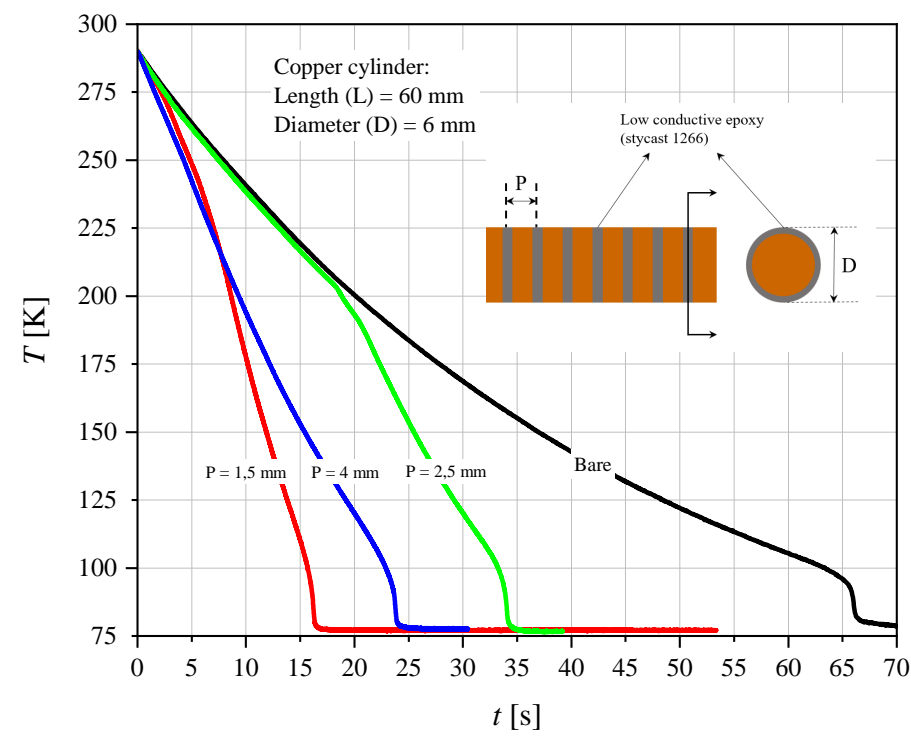

(a)

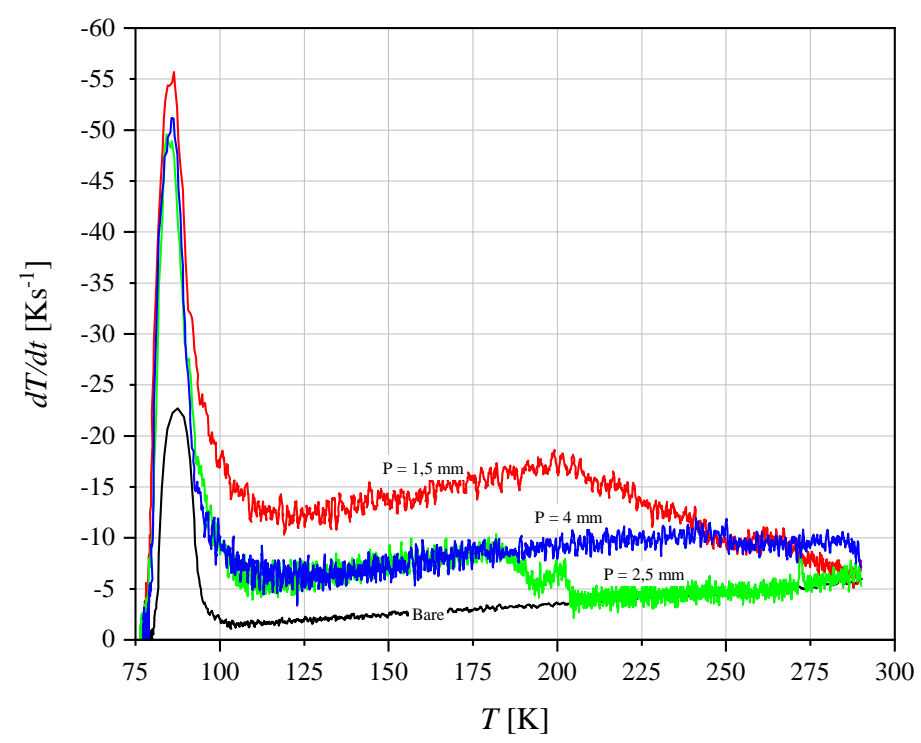

(b)

Figure (8.2) (a) - Temperature-time measurements of the bi-metallic cylinder with different pitch $(\mathrm{P})$ values. (b) - Calculated cooling rate values using temperature-time measurements. 


\section{Summary}

A good-quality frozen tissue is essential in molecular medicine for the identification of disease-associated mechanisms at molecular levels. The tissue quality relies on two critical aspects: the lag time between its excision and freezing, and the tissue cooling rate during the freezing process.

Tissue after its excision must be frozen as soon as possible to preserve the tissue quality for molecular tests. The lag time from excision to freezing depends on the logistic organizational structure within the hospital as conventional freezing procedures are not allowed in the operation theater and are therefore done at a separate location. To reduce this lag, we developed an electrically powered cryogen-free snap-freezing device, which allows tissue freezing directly at the location of its acquisition.

To help in estimating the tissue cooling rate during the freezing procedure, we also developed a detailed understanding of the heat transfer phenomena in conventional freezing procedures and the developed snap-freezer. This will help in the development of improved and precise freezing procedures.

Currently, for tissue snap-freezing, usually, a cryo-vial made of either aluminum or polypropylene is used to contain the excised tissue, which is then immersed into a coolant such as liquid nitrogen or isopentane pre-cooled using liquid nitrogen. The heat transfer at the vial-liquid interface strongly varies with the thermal properties of the vial material and the liquid thermodynamic state. The heat transfer characteristics of these procedures are not well understood. Therefore, an imprecise procedure is usually followed: immersing the vial for a random duration that varies across institutions; this makes it impossible to control the tissue cooling rate and, therefore, its quality in the frozen state. A good understanding of the heat transfer aspects of these procedures will be helpful to standardize these procedures and to develop precise procedures for improving the frozen tissue quality.

Cooling experiments performed using the conventional snap-freezing procedures show that a polypropylene vial cools faster in liquid nitrogen than in cold isopentane whereas, the opposite is true for the case of an aluminum vial. We have also shown that in liquid nitrogen, polypropylene vials exhibit an ear- 
lier transition from film to nucleate boiling compared to aluminum vials. We further developed an analytical expression to predict the temperature variation of the polypropylene vial wall and the tissue attached to it. The outcome from our work is that the difference in the cool-down time of tissue in a polypropylene vial and an empty vial is proportional to the product of the tissue heat capacity and the tube-wall thermal resistance. This enables researchers to estimate the cooling trajectory of the tissue during the cooling process allowing the development of improved snap-freezing protocols.

From the heat transfer model for polypropylene vials, it was found that during cool-down in liquid nitrogen the temperature at the liquid-solid interface can be assumed equal to the liquid temperature for materials with a thermal effusivity as low as polypropylene. This inspired us to develop an experimental approach for predicting the optimum coating thickness resulting in the minimum cool-down time for metals coated with epoxy as the low thermal effusivity material. We also verified this approach using experimental data of several copper cylinders coated with different epoxy thicknesses quenched in saturated and in sub-cooled liquid nitrogen. The optimum coating thickness reduces significantly with the degree of liquid sub-cooling. For liquid nitrogen pool temperature of $77.36 \mathrm{~K}, 74 \mathrm{~K}, 70 \mathrm{~K}$, and $66 \mathrm{~K}$, the optimum coating thickness values are measured approximately $200 \mu \mathrm{m}, 150 \mu \mathrm{m}, 100 \mu \mathrm{m}$ and $50 \mu \mathrm{m}$.

Temperature measurements of an aluminum vial during quenching in liquid nitrogen showed that the cooling rate during the film boiling regime, i.e., from room temperature till about $120 \mathrm{~K}$, is about $-25 \mathrm{Ks}^{-1}$. In literature, several authors suggested that the critical temperature region for good cell preservation in tissue is between room temperature and about $225 \mathrm{~K}$, which is the minimum supercooling temperature for water.

Using this cooling rate as a design criterion, a snap-freezer powered by a Stirling pulse tube cryocooler and consisting of a cold storage unit and heat exchangers is developed. The cryo-vial in this device is cooled by heat exchange through a tiny gas gap to a cold heat sink. We performed cooling experiments for several settings of the device, namely heat sink temperature, and gas mass flow rate in the gas gap. Experiments are performed with helium, nitrogen, and heliox (a mixture of $79 \%$ helium and $21 \%$ oxygen) in the gas gap. Cooling experiments of a vial in the snap freezing device show that cooling rates 
faster than quenching in liquid nitrogen are feasible. For the cryo-vial temperature values greater than $120 \mathrm{~K}$, the cooling rate of the cryo-vial with helium gas and a heat sink temperature of $80 \mathrm{~K}$ is faster than in liquid nitrogen; for comparison, the cool-down time of the aluminum vial from $295 \mathrm{~K}$ to $150 \mathrm{~K}$ in snap-freezer with static helium gas and $80 \mathrm{~K}$ cold-sink temperature settings is approximately $30 \%$ faster compare to the liquid nitrogen.

A one-dimensional analytical model simulating the transient interaction of the cryo-vial with the heat sink is also developed. The temperature predicted by the model for several cases agrees rather well with the measurement data. A sensitivity analysis of several parameters, namely, the gas-gap width, type of gas, heat sink temperature, and the gas flow rate, is also performed to explore the influence of these parameters on the cooling rate of the cryo-vial. Therefore, this model is a valuable tool for designing novel freezing procedures to obtain desired cooling rates for the tissue enclosed in a vial.

The preparation time for the snap-freezer is an essential parameter for its usability. This time can be estimated by predicting the cool-down of the cold heat sink, which is a copper mass of $1.25 \mathrm{~kg}$ attached to the cold end of the cryocooler. To accomplish this, the transient cooling power of the cryo-cooler needs to be known. The cryo-cooler used in the snap-freezer is a commercial off-the-shelf cryocooler manufactured by Thales Cryogenics; the only data provided in such cryocoolers is the steady-state cooling power. So in this work, we also showed a systematic approach to derive transient cooling power data by performing heating and cooling experiments. The data obtained is verified using the cooling measurements performed with the snap-freezer. We also present a method to determine the parasitic heat load in steady-state and transient operation of a commercial pulse tube cryocooler. 


\section{Samenvatting}

Een goed ingevroren weefsel is essentieel in de moleculaire geneeskunde voor de identificatie van ziekte gerelateerde mechanismen op moleculair niveau. De weefselkwaliteit is afhankelijk van twee cruciale aspecten: de tijd tussen het uitsnijden en invriezen, en de koelsnelheid van het weefsel tijdens het invriezen.

Weefsel moet na het uitsnijden zo snel mogelijk worden ingevroren om de weefselkwaliteit voor moleculaire tests te behouden. De vertragingstijd van uitsnijding tot bevriezing is afhankelijk van de logistieke organisatiestructuur binnen het ziekenhuis, aangezien conventionele bevriezingsprocedures in de operatiekamer niet zijn toegestaan en daarom op een aparte locatie moeten worden uitgevoerd. Om deze vertraging te verminderen, hebben we een elektrisch aangedreven bevriezingsapparaat vrij van cryogene substanties ontwikkeld, waarmee weefsel direct op de locatie van uitsnijding kan worden ingevroren.

Om te helpen bij het schatten van de koelsnelheid van het weefsel tijdens het invriezen, ontwikkelden we ook een gedetailleerd begrip van de warmteoverdrachtsverschijnselen in conventionele invriesprocedures en in het ontwikkelde bevriezingsapparaat. Dit zal helpen bij de ontwikkeling van verbeterde en nauwkeurige bevriezingsprocedures.

Momenteel wordt voor het snel invriezen van weefsels gewoonlijk een cryoflesje van aluminium of polypropyleen gebruikt om het weefsel in te doen, dat vervolgens wordt ondergedompeld in een koelmiddel zoals vloeibare stikstof of met vloeibare stikstof voorgekoelde isopentaan. De warmteoverdracht aan het grensvlak van het cryoflesje en de koelende vloeistof varieert sterk met de thermische eigenschappen van het materiaal van het cryoflesje en de thermodynamische toestand van de koelende vloeistof. De warmteoverdrachtseigenschappen van deze procedures zijn niet goed begrepen. Daarom wordt meestal een onnauwkeurige procedure gevolgd: het cryoflesje onderdompelen voor een willekeurige duur die varieert tussen zorginstellingen; dit maakt het onmogelijk om de afkoelsnelheid van het weefsel te regelen en daarmee ook de kwaliteit ervan in bevroren toestand. Een goed begrip van de warmteoverdrachtaspecten van deze procedures zal nuttig zijn om deze procedures 
te standaardiseren en om precieze procedures te ontwikkelen om de kwaliteit van bevroren weefsel te verbeteren.

Koelexperimenten die zijn uitgevoerd met behulp van de conventionele bevriezingsprocedures laten zien dat een polypropyleen cryoflesje sneller afkoelt in vloeibare stikstof dan in koud isopentaan, terwijl het tegenovergestelde geldt voor een aluminium cryoflesje. We hebben ook aangetoond dat polypropylene flesjes in vloeibare stikstof een eerdere overgang van het filmkook regimenaar het kernkook regime vertonen dan aluminium flesjes. We hebben verder een analytische uitdrukking ontwikkeld om de temperatuurvariatie van de polypropylene wand van het cryoflesje en het daaraan bevestigde weefsel te voorspellen. Het resultaat van ons werk is dat het verschil in koeltijd van tissue in een polypropyleen flacon en een lege flacon evenredig is met het product van de warmtecapaciteit van het weefsel en de thermische weerstand van de buiswand. Dit stelt onderzoekers in staat om het koeltraject van het weefsel tijdens het koelproces te schatten, waardoor verbeterde snap-freezing protocollen kunnen worden ontwikkeld.

Uit het warmteoverdrachtsmodel voor de polypropylene flacons bleek dat tijdens het koelen in vloeibare stikstof de temperatuur aan het vloeistof-vaste stof grensvlak gelijk kan worden gesteld aan de vloeistoftemperatuur, voor materialen met een thermische effusiviteit zo laag als polypropyleen. Dit inspireerde ons om een experimentele benadering te ontwikkelen voor het voorspellen van de optimale laagdikte zodat de koeltijd minimaal wordt voor met epoxy gecoate metalen met een lage effusiviteit. We hebben deze benadering ook experimenteel geverifieerd met behulp van verschillende koperen cilinders die zijn gecoat met verschillende epoxydiktes, ondergedompeld in verzadigde en onderkoelde vloeibare stikstof. De optimale laagdikte neemt aanzienlijk af met de mate van vloeistofonderkoeling. Voor een zwembadtemperatuur met vloeibare stikstof van $77.36 \mathrm{~K}, 74 \mathrm{~K}, 70 \mathrm{~K}$ en $66 \mathrm{~K}$, worden de optimale laagdiktewaarden gemeten ongeveer $200 \mu \mathrm{m}, 150 \mu \mathrm{m}, 100 \mu \mathrm{m}$ en 50 $\mu m$ respectievelijk.

Temperatuurmetingen van een aluminium flesje tijdens het ondergedompeld zijn in vloeibare stikstof toonden aan dat de afkoelsnelheid tijdens het filmkookregime, d.w.z. van kamertemperatuur tot ongeveer $120 \mathrm{~K}$, ongeveer $-25 \mathrm{Ks}^{-1}$ is. In de literatuur suggereerden verschillende auteurs dat het kritieke temperatuurgebied voor een goede celconservering in weefsel tussen 
kamertemperatuur en ongeveer $225 \mathrm{~K}$ ligt, wat de minimale onderkoelingstemperatuur is voor water.

Gebruikmakend van deze koelsnelheid als ontwerpcriterium, wordt een snap vriezer ontwikkeld die wordt aangedreven door een Stirling pulsbuis cryokoeler en die bestaat uit een koude opslageenheid en warmtewisselaars. Het cryoflesje in dit apparaat wordt gekoeld door warmte-uitwisseling via een kleine gasopening naar een koude warmteafleider. We hebben koelexperimenten uitgevoerd voor verschillende instellingen van het apparaat, namelijk de temperatuur van het koud reservoir en gasmassastroom in de gasopening. Er zijn experimenten uitgevoerd met helium, stikstof en heliox (een mengsel van 79\% helium en $21 \%$ zuurstof) in de gasopening. Koelexperimenten van een flesje in het bevriezingsapparaat laten zien dat de koelsnelheden sneller zijn dan de koelsnelheden haalbaar in vloeibare stikstof. Voor de cryoflesjes met een hogere starttemperatuur dan $120 \mathrm{~K}$ is de afkoelsnelheid van de cryoflacon met heliumgas en een koud reservoir van $80 \mathrm{~K}$ sneller dan bij vloeibare stikstof; ter vergelijking: de afkoeltijd van de aluminium cryoflesje van $295 \mathrm{~K}$ tot 150 $\mathrm{K}$ in een snap-vriezer met statisch heliumgas en $80 \mathrm{~K}$ temperatuurinstellingen voor de koude gootsteen is ongeveer $30 \%$ sneller in vergelijking met de vloeibare stikstof.

Er wordt ook een eendimensionaal analytisch model ontwikkeld dat de interactie van het cryoflesje met het koud reservoir over de tijd simuleert. De temperatuur die het model voor verschillende gevallen voorspelt, komt redelijk goed overeen met de meetgegevens. Een gevoeligheidsanalyse van verschillende parameters, namelijk de breedte van de gasspleet, het type gas, de temperatuur van het koellichaam en het gasdebiet, wordt ook uitgevoerd om de invloed van deze parameters op de afkoelsnelheid van het cryoflesje te onderzoeken. Daarom is dit model een waardevol hulpmiddel voor het ontwerpen van nieuwe bevriezingsprocedures om de gewenste koelsnelheden te verkrijgen voor het weefsel dat in een cryoflesje zit.

De voorbereidingstijd voor de snap-vriezer is een essentiële parameter voor zijn bruikbaarheid. Deze tijd kan worden geschat door het afkoelen van het koude reservoir te voorspellen, dit is een kopermassa van $1.25 \mathrm{~kg}$ die aan het koude uiteinde van de cryokoeler is bevestigd. Om dit te bereiken, moet het temperatuursafhankelijke koelvermogen van de cryokoeler bekend zijn. De cryokoeler die in de snap-vriezer wordt gebruikt, is een in de handel verkrijg- 
bare cryokoeler vervaardigd door Thales Cryogenics; het enige koelvermogen gerelateerde gegeven die met dergelijke cryokoelers worden meegeleverd, is het koelvermogen in stationaire toestand. Daarom laten we in dit werk ook een systematische benadering zien om de temperatuur afhankelijke koelvermogensgegevens af te leiden door verwarming en koelingsexperimenten uit te voeren. De verkregen gegevens worden geverifieerd met behulp van de koelingsmetingen die zijn uitgevoerd met de snap-vriezer. We presenteren ook een methode om de parasitaire warmtebelasting te bepalen in de stationaire en afkoelende toestand van een commerciële pulsbuis-cryokoeler. 


\section{Acknowledgment}

I am very fortunate to have many people who supported me throughout my journey of finishing this thesis. I would like to start by thanking my supervisor Srinivas Vanapalli (Srini) and my promotor Marcel ter Brake for their continuous support and guidance during my Ph.D.

Srini, first of all, I thank him for introducing me to this beautiful country of tulip gardens, windmills, museums, and seductive Stroop waffles. I have to say that any amount of thanks would not be enough for his immense support and inspiration on my way through this unpredictable Ph.D. journey. It was his mentorship that was extremely helpful for me in making a smooth transition from having an engineering approach to think more scientifically. Apart from a good mentor, he is a wonderful human being and a kind-hearted person, who is always there for everyone in need. I learned a lot from him both professionally as well as personally. I am also looking forward to working with him in the future.

Marcel, it was a great pleasure to work with him. I really enjoyed having many of our conversations in the group meetings and over the coffee table. I am greatly thankful for his continuous feedback on my research progress, publications, and thesis. I also appreciate his contribution to maintaining a healthy and learning atmosphere at the EMS. To my knowledge, he will be retiring in few years from his professional life and perhaps I might not be there to say goodbye to him in person. So, I wish him great success for his remaining tenure at the EMS and also wish him a very happy and healthy life ahead.

Harry Holland, I would like to thank him for all his help in the EMS lab, without his help I could not imagine finishing my experimental work in time. I really enjoyed having many brainstorming sessions, building up experimental setups, working together on research papers with him. I certainly appreciate his creative mindset and hands-on experience in building up experimental setups. With saying thanks again, I wish him a happy and healthy life ahead. 
Cris Vermeer, I really appreciate his help in the lab, especially during the first two years of my Ph.D. work. He is such a charming and positive personality who certainly gives the impression of an ideal colleague. I thank him for arranging all those trips with the group, it was really fun going out with the EMS group to enjoy many activities together.

Ans Veenstra, I thank her for facilitating my transition to the Netherlands. All her help from managing funds to arranging stuff for many things is really appreciated.

Abhishek Purandare, although we did not share much of our time working together, but from a very short experience with him, I am very certain that he is a very helpful and kind-hearted person. I would like to thank him for all our time spent in the socio-corner and also wish him great success with his Ph.D. work.

Nando Tolboom, probably the most annoying person in the EMS group but still is a fun-loving and very helpful person to have around. He certainly possesses a very high noise to the volume (body) ratio and is probably the loudest dutch person amongst all I met during my Ph.D. It was a fun experience sharing my working space with him, I wish him great success with his Ph.D. work and also for his future ambitions.

Koen Ledeboer is a very helpful person, working together with him at EMS was really fun. He is probably the most humble Dutch person I met during the course of my Ph.D. One thing that I can tell about him is that if you have a plan just call him and he will be ready to go. I really enjoyed having our multiple trips together around Europe.

Other than these, I would like to thank all other people of the EMS for maintaining a very healthy atmosphere for anyone to work in. Thanks, Peng, Jaap, Marc, Gonçalo, Anne, Jeroen, Sander, Ruben, Hidde, Jianfeng, Tiemo, Anvar, Erik, Arend, Koen Lotze, Fabrice, Secil, Tommaso, Nir, Konstantin, Haishan, Izak, Bas, Thomas, Teun, Simon, Peter, Rick, Steven, Keyang for making my stay at the University of Twente a great and memorable experience. 
I also would like to thank our project partners at UMC Amsterdam - Mariette, Henk Verheul, Henk Dekker, Iris, Hanneke. Sharing our work together in the project user-committee meetings was really insightful. Furthermore, I would like to extend my gratitude to all the user committee members - Peet, Hans, Mathijs, Roel, Theo, for their great insights into the progress of the project.

Thank you very much, NWO for providing the funding for this project and also to Linda, Elisa for organizing user committee meetings.

I would also like to acknowledge the contribution of my previous mentor Prof. Milind Atrey for laying the foundation of cryogenics for me. It was his lectures when I was first introduced to the field of cryogenics. Working together with him was a great learning experience, where I was encouraged to get my hands dirty in the lab.

Now, focusing on my personal life - I would like to thanks all my friends who made my stay very memorable from the very first day in Enschede, Netherlands. Furthermore, I would like to give a special mention to some of my close friends for their great support and our experiences together.

Arka Bhakta, also known as "B..... waale Chacha". Well, not a kind of Bhakta (devotee) you want to meddle with. I am really thankful to him for his hospitality at his apartment in Mooienhof, Enschede that made many of my weekends memorable. He is the chef, driver, and certainly the charm of our group and I won't hesitate to shout out that "You can't cook better Biryani than Arka". At last, I would like to say that although we both tend to have many strong disagreements over many things, I really respect him for his unconventional thinking. It is my great pleasure to have such a friend, who can be called anytime without hesitation.

Jagadeesh Narayana Swamy, also known as "Dude Ji". Say hello to the "Trevor Noah" of our group. He is factually the most loveable person, we have in our group. I am greatly thankful to him for bringing a fusion of sarcasm, humor, and logic to all our conversations, and also for sharing great memes and gifs in our group. He certainly unleashes his creativity in the WhatsApp groups and deserves to be known as "Meme Master". Apart from being a horrible chef, he is a very kind, helpful, and down-to-earth person, who you would really like to be a friend with for the rest of your life. I wish him great 
success with his Ph.D. work and also other future aspirations.

Sugandh Chauhan, also known as "Madam Ji" - the girl who is famous for her love for food and dance. Well, you can hate her for some of the stupidity she does, but I can guarantee that you will eventually fall for her, for the same later on. She is the "Lady Dhoni" of our group, who knows how to finish things in style, whether it is her Ph.D. or her badminton match. You can't beat her in the game of dance, badminton, and time management. I am really thankful to her for organizing almost every party that we had in our group.

Florentina Badea, also known as "Flory Didi". Okay, let me start with a humble request to the Indian government - "Please allow her to carry dual nationality". Oh god, she really surprises me with her love for India. She is certainly a very ambitious and outspoken girl. I am really thankful to her for bringing laughter and dance moves to our parties together. I also wish her great success in becoming a great influencer in the coming days.

Nidale Tarhate Chihab, also known as "Habibi". Although we only met a couple of times during my Ph.D., she certainly made herself a special place. She is the fashion icon of our group and is known for her expensive clothes and spontaneity. She is definitely a person that you can count on except if you are sharing a drive with her. She is an excellent host and carries an abundance of positive energy with her. I am very fortunate to have such a person in my life, looking forward to having more trips together with her.

I would like to conclude this by giving a special mention to the person without whom my thesis writing would have been a dull experience. Parni Nijhawan is one of the most kind-hearted and sweetest people I know in my life. I am really thankful to her for all our conversations that made me feel better after draining all my energy during the thesis writing. Apart from being a good friend, she is a wonderful human being. With this, I would like to extend my good wishes for her Ph.D. thesis and also with the follow-ups. 


\section{About the author}

Sahil was born on the 22nd October 1991, in the Yamunanagar district of Haryana, India. From a very young age, he was interested in solving math puzzles and was often challenged by his father in doing so. The analytical mindset that he was able to develop while growing up helped him to decide on pursuing engineering as a career.

For his bachelor's, he decided to follow the mechanical engineering program at DCRUST Murthal in India, where he found himself attracted to the applications of thermodynamic cycles in commonly used household appliances. This led him to explore more on this path and decided to persue his master's program in thermal and fluid engineering at the Indian Institute of Technology (IIT) Bombay, India. His specific interest in cryogenics emerged from attending a course on cryogenics engineering during the master's program. Following this, he pursued his master thesis under the supervision of Prof. Milind Atrey at the cryogenic engineering lab, IIT Bombay; for which, he developed a single-stage Stirling-type pulse tube cryocooler. For his Ph.D., he joined the Applied Thermal Sciences Lab led by Srinivas Vanapalli, which is part of the Energy, Materials and Systems cluster at the University of Twente, Netherlands. During his Ph.D., he worked on many systems that include single and two-phase heat transfer. He also developed very good modeling skills in capturing the transient thermal behavior of many complex systems.

Apart from his studies, he is an excellent athlete and likes to maintain a healthy and balanced lifestyle. He is also a very good bhangra dancer and likes playing chess. He considers himself inclined towards spirituality and seeking to explore it further in the future. 


\section{Bibliography}

[1] S. N. Bhat, A. Sharma, S. Bhat, Vitrification and glass transition of water: Insights from spin probe esr, Physical Review Letters 95 (23) (2005) 235702 .

[2] "Fatherhood After Death Has Now Been Proved Possible". Cedar Rapids Gazette. April 9, 1954.

[3] E. J. C. Polge, Low-temperature storage of mammalian spermatozoa, Proceedings of the Royal Society of London. Series B-Biological Sciences 147 (929) (1957) 498-508.

[4] U. Desa, World population prospects 2019: Highlights, New York (US): United Nations Department for Economic and Social Affairs (2019).

[5] S. P. Naber, Continuing role of a frozen-tissue bank in molecular pathology., Diagnostic molecular pathology: the American journal of surgical pathology, part B 5 (4) (1996) 253-259.

[6] J. A Fairley, K. Gilmour, K. Walsh, Making the most of pathological specimens: molecular diagnosis in formalin-fixed, paraffin embedded tissue, Current drug targets 13 (12) (2012) 1475-1487.

[7] S.-R. Shi, C. Liu, L. Pootrakul, L. Tang, A. Young, R. Chen, R. J. Cote, C. R. Taylor, Evaluation of the value of frozen tissue section used as "gold standard" for immunohistochemistry, American journal of clinical pathology 129 (3) (2008) 358-366.

[8] W. Tang, Z. Hu, H. Muallem, M. L. Gulley, Quality assurance of rna expression profiling in clinical laboratories, The Journal of molecular diagnostics 14 (1) (2012) 1-11.

[9] S. Mareninov, J. De Jesus, D. E. Sanchez, A. B. Kay, R. W. Wilson, I. Babic, W. Chen, D. Telesca, J. J. Lou, L. Mirsadraei, et al., Lyophilized brain tumor specimens can be used for histologic, nucleic acid, and protein analyses after 1 year of room temperature storage, Journal of neurooncology 113 (3) (2013) 365-373. 
[10] A. von Teichman, M. Storz, S. Dettwiler, H. Moch, P. Schraml, Whole genome and transcriptome amplification: practicable tools for sustainable tissue biobanking?, Virchows Archiv 461 (5) (2012) 571-580.

[11] S. Leonard, J. Logel, D. Luthman, M. Casanova, D. Kirch, R. Freedman, Biological stability of mrna isolated from human postmortem brain collections, Biological psychiatry 33 (6) (1993) 456-466.

[12] T.-Y. Chu, K.-S. Hwang, M.-H. Yu, H.-S. Lee, H.-C. Lai, J.-Y. Liu, A research-based tumor tissue bank of gynecologic oncology: characteristics of nucleic acids extracted from normal and tumor tissues from different sites, International Journal of Gynecologic Cancer 12 (2) (2002).

[13] S. Mager, M. H. Oomen, M. M. Morente, C. Ratcliffe, K. Knox, D. J. Kerr, F. Pezzella, P. H. Riegman, Standard operating procedure for the collection of fresh frozen tissue samples, European Journal of Cancer 43 (5) (2007) 828-834.

[14] S. Steu, M. Baucamp, G. von Dach, M. Bawohl, S. Dettwiler, M. Storz, H. Moch, P. Schraml, A procedure for tissue freezing and processing applicable to both intra-operative frozen section diagnosis and tissue banking in surgical pathology, Virchows Archiv 452 (3) (2008) 305-312.

[15] J. O. Karlsson, M. Toner, Long-term storage of tissues by cryopreservation: critical issues, Biomaterials 17 (3) (1996) 243-256.

[16] D. H. Jackson, R. E. Banks, Banking of clinical samples for proteomic biomarker studies: A consideration of logistical issues with a focus on pre-analytical variation, PROTEOMICS-Clinical Applications 4 (3) (2010) 250-270.

[17] (accessed on July 15, 2020). [link].

URL https://www.thermofisher.com/order/catalog/product/ 5100-0001\#/5100-0001

[18] (accessed on July 15, 2020). [link]. URL https://ww .brookslifesciences.com/products/ coolrack $-96 \times 1 \mathrm{ml}$ 
[19] (accessed on July 15 , 2020). [link].

URL https://www.spscientific.com/Products/Thermal_ Products/FTS_Systems/Low_Temperature_Baths/HistoChill_ Tissue_Freezing_Bath/

[20] (accessed on July 15, 2020). [link].

URL https://www. leicabiosystems. com/histology-consumables/sectioning/ neuroscience-solutions/frozen-tissue-preparation/ products/leica-gentle-jane/

[21] L. A. Bromley, Heat transfer in stable film boiling, Vol. 2295, US Atomic Energy Commission, Technical Information Division, 1949.

[22] B. P. Breen, Effect of diameter of horizontal tubes of film boiling, Ph.D. thesis, University of Illinois at Urbana-Champaign (1961).

[23] V. Dhir, J. Lienhard, Laminar film condensation on plane and axisymmetric bodies in nonuniform gravity (1971).

[24] A. Sakurai, M. Shiotsu, K. Hata, Correlations for subcooled pool film boiling heat transfer from large surfaces with different configurations, Nuclear engineering and design 120 (2-3) (1990) 271-280.

[25] W. J. Wafer, The effect of diameter on the film boiling behavior of liquid nitrogen (1971).

[26] E. Le Fevre, A. Ede, Laminar free convection from the outer surface of a vertical circular cylinder, in: Proceedings of the 9th International Congress Applied Mechanics, Vol. 4, 1956, pp. 175-183.

[27] P. Wang, R. Kahawita, D. Nguyen, Transient laminar natural convection from horizontal cylinders, International journal of heat and mass transfer 34 (6) (1991) 1429-1442.

[28] C. Beduz, R. Scurlock, A. Sousa, Angular dependence of boiling heat transfer mechanisms in liquid nitrogen, in: Advances in cryogenic engineering, Springer, 1988, pp. 363-370.

[29] F. Nix, D. MacNair, The thermal expansion of pure metals: copper, gold, aluminum, nickel, and iron, Physical Review 60 (8) (1941) 597. 
[30] B. McBride, S. Gordon, M. Reno, Nasa technical paper 3287 (1993).

[31] C. Y. Ho, R. W. Powell, P. E. Liley, Thermal conductivity of the elements, Journal of Physical and Chemical Reference Data 1 (2) (1972) 279-421.

[32] J. Grebowicz, S.-F. Lau, B. Wunderlich, The thermal properties of polypropylene, in: Journal of Polymer Science: Polymer Symposia, Vol. 71, Wiley Online Library, 1984, pp. 19-37.

[33] G. Hartwig, Polymer properties at room and cryogenic temperatures, Springer Science \& Business Media, 2013.

[34] M. Sansinena, M. Santos, N. Zaritzky, R. Baeza, J. Chirife, Theoretical prediction of the effect of heat transfer parameters on cooling rates of liquidfilled plastic straws used for cryopreservation of spermatozoa, CryoLetters 31 (2) (2010) 120-129.

[35] Z. Jiang, G. Zhao, S. C. Hossain, D. Gao, Coupled experimentalmodeling analyses of heat transfer in ex-vivo vs55-perfused porcine hepatic tissue being plunged in liquid nitrogen for vitreous cryopreservation, International Journal of Heat and Mass Transfer 106 (2017) 970-979.

[36] T. Wang, G. Zhao, H. Tang, Z. Jiang, Determination of convective heat transfer coefficient at the outer surface of a cryovial being plunged into liquid nitrogen, CryoLetters 36 (4) (2015) 285-288.

[37] S. Li, Y. Xie, X. Wu, Hardness and toughness investigations of deep cryogenic treated cold work die steel, Cryogenics 50 (2) (2010) 89-92.

[38] H. Hu, J. N. Chung, S. H. Amber, An experimental study on flow patterns and heat transfer characteristics during cryogenic chilldown in a vertical pipe, Cryogenics 52 (4-6) (2012) 268-277.

[39] D. Takeda, K. Fukiba, H. Kobayashi, Improvement in pipe chilldown process using low thermal conductive layer, International Journal of Heat and Mass Transfer 111 (2017) 115-122.

[40] F. Moreaux, J. Chevrier, G. Beck, Destabilization of film boiling by means of a thermal resistance, International Journal of Multiphase Flow 2 (2) (1975) 183-190. 
[41] C. Cowley, W. Timson, J. Sawdye, A method for improving heat transfer to a boiling fluid, Industrial \& Engineering Chemistry Process Design and Development 1 (2) (1962) 81-84.

[42] J. Maddox, T. Frederking, Cooldown of insulated metal tubes to cryogenic temperatures, in: Advances in Cryogenic Engineering, Springer, 1966, pp. 536-546.

[43] L. Manson, A periodic nonuniform heat-transfer mechanism in film boiling (1967).

[44] Y. Kikuchi, T. Hori, I. Michiyoshi, Minimum film boiling temperature for cooldown of insulated metals in saturated liquid, International journal of heat and mass transfer 28 (6) (1985) 1105-1114.

[45] A. Bejan, Heat transfer, John Wiley \& Sons, Inc., 1993. URL https : //books . google.nl/books?id=TgtRAAAAMA J

[46] V. Dhir, Boiling heat transfer, Annual review of fluid mechanics 30 (1) (1998) 365-401.

[47] J. Bernardin, I. Mudawar, The leidenfrost point: experimental study and assessment of existing models (1999).

[48] (accessed on June 07, 2021). [link].

URL https://www.fkit.unizg.hr/_download/repository/H1_ IMPACT_OF_THERMAL_DIFFUSIVITY_AND_EFFUSIVITY__-__VAZNO_ ---. pdf

[49] S. Hellmann, M. Noe, Influence of different surface treatments on the heat flux from solids to liquid nitrogen, IEEE transactions on applied superconductivity 24 (3) (2013) 1-5.

[50] A. Tsoi, A. Pavlenko, Enhancement of transient heat transfer at boiling on a plate surface with low thermoconductive coatings, Thermophysics and Aeromechanics 22 (6) (2015) 707-712.

[51] D. He, P. Zhang, F. Lv, S. Wang, D. Shu, Cryogenic quenching enhancement of a nanoporous surface, International Journal of Heat and Mass Transfer 134 (2019) 1061-1072. 
[52] T. Olewski, L. Véchot, M. Mannan, Study of the vaporization rate of liquid nitrogen by small-and medium-scale experiments, CHEMICAL ENGINEERING 31 (2013).

[53] T. Olewski, S. Mannan, L. Véchot, Validation of liquid nitrogen vaporisation rate by small scale experiments and analysis of the conductive heat flux from the concrete, Journal of Loss Prevention in the Process industries 35 (2015) 277-282.

[54] M. Cha, X. Yin, T. Kneafsey, B. Johanson, N. Alqahtani, J. Miskimins, T. Patterson, Y.-S. Wu, Cryogenic fracturing for reservoir stimulationlaboratory studies, Journal of Petroleum Science and Engineering 124 (2014) 436-450.

[55] R. Li, Z. Huang, G. Li, X. Wu, P. Yan, Study of the conductive heat flux from concrete to liquid nitrogen by solving an inverse heat conduction problem, Journal of Loss Prevention in the Process Industries 48 (2017) $48-54$.

[56] P. Chin, J. Hwang, T. Lin, The mechanism of heat transfer in transition boiling, International journal of heat and mass transfer 32 (7) (1989) 1337-1349.

[57] D. W. Hahn, M. N. Özisik, Heat conduction, John Wiley \& Sons, 2012.

[58] J. Chung, J. Dong, H. Wang, S. Darr, J. Hartwig, Enhancement of convective quenching heat transfer by coated tubes and intermittent cryogenic pulse flows, International Journal of Heat and Mass Transfer 141 (2019) 256-264.

[59] J. Chung, S. Darr, J. Dong, H. Wang, J. Hartwig, Heat transfer enhancement in cryogenic quenching process, International Journal of Thermal Sciences 147 (2020) 106117.

[60] (accessed on November 14, 2019). [link]. URL http://www.cmr-direct.com/en/cmr-epoxy-1266-1kg

[61] S. Nakamura, T. Fujii, S. Matsukawa, M. Katagiri, H. Fukuyama, Specific heat, thermal conductivity, and magnetic susceptibility of cyanate 
ester resins-an alternative to commonly used epoxy resins, Cryogenics 95 (2018) 76-81.

[62] M. T. Hepworth, C. O. Ruud, Viscosity and density of liquid copper and copper alloys, INCRA REP (1974).

[63] F. Demiray, J. Kim, Microscale heat transfer measurements during pool boiling of fc-72: effect of subcooling, International Journal of Heat and Mass Transfer 47 (14-16) (2004) 3257-3268.

[64] R. W. Schafer, What is a savitzky-golay filter?[lecture notes], IEEE Signal processing magazine 28 (4) (2011) 111-117.

[65] T. Inoue, N. Kawae, M. Monde, Effect of subcooling on critical heat flux during pool boiling on a horizontal heated wire, Heat and mass transfer 33 (5-6) (1998) 481-488.

[66] T. L. Bergman, F. P. Incropera, D. P. DeWitt, A. S. Lavine, Fundamentals of heat and mass transfer, John Wiley \& Sons, 2011.

[67] G. Liang, I. Mudawar, Pool boiling critical heat flux (chf)-part 1: Review of mechanisms, models, and correlations, International Journal of Heat and Mass Transfer 117 (2018) 1352-1367.

[68] M. Arik, A. Bar-Cohen, Effusivity-based correlation of surface property effects in pool boiling chf of dielectric liquids, International Journal of Heat and Mass Transfer 46 (20) (2003) 3755-3764.

[69] J. B. Galvin, F. Marashi, 2-methylbutane (isopentane), Journal of Toxicology and Environmental Health Part A 58 (1-2) (1999) 23-33.

[70] S. Vanapalli, B. Colijn, C. Vermeer, H. Holland, T. Tirolien, H. J. ter Brake, A passive, adaptive and autonomous gas gap heat switch, Physics procedia 67 (2015) 1206-1211.

[71] S. Vanapalli, R. Keijzer, P. Buitelaar, H. J. ter Brake, Cryogenic flatpanel gas-gap heat switch, Cryogenics 78 (2016) 83-88.

[72] M. A. van Limbeek, S. Vanapalli, Thermal conductance of interleaving fins, Physical Review Applied 12 (6) (2019) 064048. 
[73] Nist webbook, http://wtt-pro.nist.gov/wtt-pro/.

[74] E. Mason, S. Saxena, Approximate formula for the thermal conductivity of gas mixtures, The Physics of fluids 1 (5) (1958) 361-369.

[75] A. Wassiljewa, Warmeleitung in gasgemischen, Physik. Z. 5 (1904) 737742.

[76] P. Mazur, Freezing of living cells: mechanisms and implications, American Journal of Physiology-Cell Physiology 247 (3) (1984) C125-C142.

[77] A. Comsol, Comsol multiphysics® v. 5.2, Stockholm, Sweden (2016).

[78] M. A. van Limbeek, S. Jagga, H. Holland, K. Ledeboer, M. ter Brake, S. Vanapalli, Cooling of a vial in a snapfreezing device without using sacrificial cryogens, Scientific reports 9 (1) (2019) 1-9.

[79] R. C. Dhuley, S. Posen, M. I. Geelhoed, O. Prokofiev, J. C. T. Thangaraj, First demonstration of a cryocooler conduction cooled superconducting radiofrequency cavity operating at practical $\mathrm{cw}$ accelerating gradients, Superconductor Science and Technology 33 (6) (2020) 06LT01. doi : $10.1088 / 1361-6668 / a b 82 f 0$.

URL https : //doi .org/10.1088\%2F1361-6668\%2Fab82f0

[80] R. C. Dhuley, R. Kostin, O. Prokofiev, M. I. Geelhoed, T. H. Nicol, S. Posen, J. C. T. Thangaraj, T. K. Kroc, R. D. Kephart, Thermal link design for conduction cooling of srf cavities using cryocoolers, IEEE Transactions on Applied Superconductivity 29 (5) (2019) 1-5.

[81] C. Liubiao, Z. Qiang, Z. Xiaoshuang, Z. Yuan, W. Junjie, An optical cryostat for use in microscopy cooled by stirling-type pulse tube cryocooler, Physics Procedia 67 (2015) 354 - 359, proceedings of the 25th International Cryogenic Engineering Conference and International Cryogenic Materials Conference 2014. doi:https://doi.org/10.1016/j.phpro.2015.06.040.

URL http://www.sciencedirect.com/science/article/pii/ S1875389215004216

[82] R. Arts, T. Benschop, P. Bruins, T. Rijks, G. de Jonge, T. Trollier, Development of cost-effective cryocoolers for space, Cryocoolers 18 (2014) 595-600. 
[83] G. Grossman, P. E. Bradley, M. A. Lewis, R. Radebaugh, Model for transient behavior of pulse tube cryocooler, Cryogenics 51 (3) (2011) 124-131.

[84] S. Vanapalli, High-frequency operation and miniaturization aspects of pulse-tube cryocoolers (2008).

[85] S. Vanapalli, M. Lewis, G. Grossman, Z. Gan, R. Radebaugh, H. t. Brake, Modeling and experiments on fast cooldown of a $120 \mathrm{hz}$ pulse tube cryocooler, in: AIP Conference Proceedings, Vol. 985, American Institute of Physics, 2008, pp. 1429-1436.

[86] G. K. White, S. Collocott, Heat capacity of reference materials: $\mathrm{Cu}$ and w, Journal of physical and chemical reference data 13 (4) (1984) 12511257.

[87] N. Simon, E. Drexler, R. Reed, Properties of copper and copper alloys at cryogenic temperatures. final report, Tech. rep., National Inst. of Standards and Technology (MSEL), Boulder, CO (United ... (1992).

[88] Y. S. Touloukian, C. Y. Ho, Thermophysical properties of selected aerospace materials. part 2. thermophysical properties of seven materials, Tech. rep., THERMOPHYSICAL AND ELECTRONIC PROPERTIES INFORMATION ANALYSIS CENTER ... (1977).

[89] M. Shabihkhani, G. M. Lucey, B. Wei, S. Mareninov, J. J. Lou, H. V. Vinters, E. J. Singer, T. F. Cloughesy, W. H. Yong, The procurement, storage, and quality assurance of frozen blood and tissue biospecimens in pathology, biorepository, and biobank settings, Clinical biochemistry 47 (4-5) (2014) 258-266.

[90] (accessed on July 15 , 2020). [link].

URL https://www.linde-cryo.nl/nl/

producten/dryshipper-voor-sample-transport/

dryshipper-voor-sample-transport.html

[91] (accessed on July 15, 2020). [link].

URL https://www.brookslifesciences.com/products/ cryopod-carrier 
[92] M. M. Rahman, J. Pollack, M. McCarthy, Increasing boiling heat transfer using low conductivity materials, Scientific reports 5 (2015) 13145. 